\title{
Discrete-Coil Investigations of Modular Stellarator Configurations
}

\author{
C. G. Lilliequist
}

\section{DISCLAIMER}

\begin{abstract}
This report was prepared as an account of work sporsored by an agency of the United States Government. Neither the United St ttes Government nor any agency thereuf, nor any of their employes. makes any warranty, express or implied, or assumes any legal liability or responsibllity for the accuracy, completeness, or usefulness of any information, apparatus, product, or prosess disclosed, or represents that it: use would not infringe privately owned rights. Reference herein to iny specific commercial product, process, or service by trade name, trademark, manufacturer, or otherwise does not niscessarily constitute or imply its endorsement, recommendation, or favoring by the United States Government or any agency thereof. The views and opinions of authors expressed herein do not necessarily state of reflect those of the United States Governmznt or any agency therenf.
\end{abstract}


DISCRETE-COIL INVESTIGATIONS OF MODULAR STELLARATOR CONFIGURATIONS

by

C. G. Lilliequist

\begin{abstract}
The existence of a vacuum magnetic well is generally accepted as a prerequisite to start-up. The special set of modular-stellarator configurations selected for the present computational investigation were derived from coefficients and equations that produced finite-beta wells in a continuous-current-sheet representation. The corresponding coils did not produce nagnetic wells in a vacuum when their field configuration was investigated with a discrete-coil code. Vacuiam magnet $\mathrm{c}$ wells have been identified through the use of this discrete-coil code in previous Heliac and stellarator studies. Therefore, these finite-beta magnetic wells could be the consequence of the continuous-current-sheet model: a conjecture that is supported by the linear scaling of the magnetic hills found in the present work as a function of the separation between the coils used. In addition to magnetic field profiles, comparisons are shown here of ripple, rotational transferm, and flux-surface shapes for the discrete-coil, modular stellarators under study lnitially, each of the significant parameters affecting the shape of the control surface upon which the colls lie and the dejormation of the individuel coils was varied separately in search of a vacuun magnetic well in the parameter neighborhood of the successful finite-beta configuration. The magnetic hills persisted in vacuum ihroughout the parameter search even with many coils per field period, thereby approaching the continuous-sheet-current limit. The implication seems to be either that it is computationelly impossible to increase the number of colls per field period sufficiently to reproduce the magnetic field of the continuous-sheet-current models, that good flux surfaces dont't exist in the discreie-cojl field configurations, or tinat the plasma is somehow responsible for the magnetic well.
\end{abstract}




\section{INTRODUCTION}

A study by McNamara et al $\underline{a}^{1}$ of magnetic well properties in a straight-axis helical geometry, utilizing a stream-function formulation of the magnetıc flelds, revealed a varıety of magnetıc wells near the magnetic axis and the separatrix. Of necessity, this approach could not account for the effects oi toroidicity and discrete coils. Their hellcal system suggested two tests for the existence of a well: (1) evaluation of $V^{\prime \prime}$ at the magnetic axis ( $V$ " refers to the second derivative of plasma volume with respect to magnetic $f(u x)$ and (2) the ratio of $\int d l / B$ on axis to its value at the separatrjx. Both tests work for simple monotonic magnetic-field profiles but not for more complex ones. They acknowledged some inadequacies in their ratio test, even in a simple helical geometry. Wells near the separatrix identified with the stream-function treatment were narrowly confined to the immediate vicinity of the separatrix. wh, ch would make them extremely difficult to detect with a more realistic discrete-coll code owing to the enhancement of ripple near the coll and its effect on $\int d l / w$. Their general conclusion for helical systems without a central conductor was that wells do exist, but that such systems might require careful surface by surface numerical evaluation to recognize and conflrm their existence. In other words, the well depth may not be a monotonic function of the distance from the magnetic axis. And the existeice of wells in the sense of $V^{\prime \prime}$ depends on the topology of the magnetic surfaces.

One of the assertions made by Greene and Johnson ${ }^{2}$ as a consequence of their analysis of ideal hydromagnetic interchange instabilities was that a plasma could not dig its own well. Their magnetic field model had nested toroidal flux surfaces consistent with that produced by a continuols-sheet-current system.

The vacuum fleld line tracing code. TORSiDo. has been used recently to test the reactor sultability of numerous toroldal discrete-coil systeins ${ }^{3}$ is TORSIDO, the magnetic field at a point is computed by application of the Blot-Savart law to the sum of all the contributions from ell parts of each finite cross-sectional current element. Individual magnetic-field lines are then traced and a puncture plot of the flux surface is produced. These modular stellarator investigations w:th TORSIDO and a different coil-winding law than used in the present program discovered a slight magnetic well (less than $G 5 \%$ in $<B=2$. see Appendix A) in a reactor-compatible configuration with 
coils of circular bore, nine colls per field period, two poloidal fleld periods, four toroidal field periods in total, and an aspect ratio of $\mathrm{six}(\mathrm{A}=$ 6). A decrease in the aspect ratio for this modular stellarator configuration caused the well depth to increase: between $A=6.9$ and $A=4.4$ the $\langle B\rangle^{2}$-well was less than $0.3 \%$, at $A=4.4 i i$ was $1.1 \%$ and at $A=2.5$ it was $6.6 \%$. Unfortunately, the smaller coil aspect ratios have larger increments in poloidal phase between adjacent coils, accompanied by larger ripple $/ \varepsilon \equiv\left(B_{\max }\right.$ $\left.\left.-B_{\min }\right) /\left(B_{\max }+B_{\min }\right)\right)$, as well as poor volume utilization. The $\langle B\rangle^{2}$-well in this configuration also increased in depth with decreasing lateral distortion, where products of the number of coils and the current and of the number of coils and the lateral distortion coefflcient were kept constanl to avoid the masking effects of changing coil spacing and magnetic-field strength. A decrease in the lateral deformation to the limit of planar coils would completely eliminate the presence of an effective hardcore. The trends in magnetic-field profiles, ripple, and island formation for the modular stellarators developed with this coil-winding law were generally confirmed by the present investigation, if one equates reduction of magnetic hill to increase of magnetic-well depth.

The TORSIDO vacuum magnetic-field-line-tracing code was chosen for the present analysis because of its speed and simplicity, its ready adaptation to different coil-wirding laws, and its accuracy against benchmarks. 3 This code was applied to a family of modular-stellarator configurations closely reiated to those showing magnetic wells in a previous finite-beta, continuous-current-sheet analysis. ${ }^{+}$The primary goal of the ensuing investigation was the determination of whether or not such wells persist in a vacuun. This investigation was restricted to magnetic wells on axis. More complex magnetic-field profiles with wells near the separatrix could exist. but they would be difficult to recognize in a discrete-coil analysis. Mll modular stellarators in this study were established with the same coil-winding law and surlace equations. Varlations in shepe, spacing, and orientation of the coils were accomplished through sultable choices of coefficients in these equations. The modular, nonplanar coils were treated in the code as finite cross-section conductors, uniformly separated in toroidal angle.

\footnotetext{
+Information provided by 0 . Betancourt, New York University/Courant lnstitute, 251 Mercer Street, New York, NY 10012.
} 
The first part of the investigation dealt with variations of the individual parameters from those that produced nested flux surfaces of uniformly spaced field lines and magnetic wells in finite beta. Although flux surfaces of this quelity were found, their shapes were not the same and no magnetic wells were found. The second part of the program dealt with multiple-parameter searches for a family of flux-surface profiles that would lead to a magnetic well. Such multiple-pacaneter alterations could lead to coil configurations considerably different from those of the finite-beta colls

In the present vecuum modular stellarator study, the position and shape of the discrete colls were determined by coll-winding and control surface equations or iginaily derived by Betancourt for a finite beta, fully toroidal computation in a continuous-current-sheet approximation of finite coils. Specifically, the equation for the control-surface upon which the coils were wound was taken from the geieral control-surface equation used in those stellarator and hi:iac studies.

$$
\left.r+1 z=\mid \Delta_{1}^{*}+r_{b}^{*}\left(1+\Delta_{2}{ }^{*} \cos 2 \pi u\right) e^{1 C} \sin 2 \pi u\right] e^{-2 \pi 1 v}
$$

The coefficients of this equation represent the distance beyond the end of the major radius to the surface: (or coll's) center $\left(\Delta_{1}{ }^{*}\right.$ ), the surface's average radius (or radial widih) $\left(\mathrm{r}_{\mathrm{b}}{ }^{*}\right)$ and height $\left(\Delta_{2}{ }^{*}\right)$, and its concavily (C) (Fig. 1). (Starting vaiues suggested by Betancourt from the high-beta equilibrium were $\Delta_{1}{ }^{\prime}=-0.3, \mathrm{r}_{\mathrm{b}}{ }^{\prime}=1.09, \Delta_{2}{ }^{\prime}=0.7$. and $\mathrm{C}=1.2$. These valies produced a cortrol-surface with a D-shaped cross section).

The coll-winding law was derived from the equation for the boundary value of the scalar potential. $\Phi$, from Betancourt's continuous-current-sheet model.

$$
\Phi=c_{1} v+c_{2} z+c_{3} \tan ^{-1}\left(c_{4} \sin 2 \pi u /\left(1+c_{4} \cos 2 m\right)\right)+c_{5} \sin 2 \pi u .
$$

The coeffacients of this equation represent toroidal magnetic field $\left(c_{1}\right)$,

\footnotetext{
${ }^{++}$Information provided by 0 . Betancourt. New York University/Courant institute, 251 Mercer Street. New York, NY 10012 . 
uniform vertical magnetic field $\left(c_{2}\right)$, lateral deformation $\left(c_{3}\right.$ and $\left.c_{4}\right)$, and coil tilt from vertical $\left(c_{5}\right)$. (The high-beta sterting values for these coefficients were $c_{1}=12.25, c_{2}=0.0, c_{3}=1.2, c_{4}=0.625$ and $c_{5}=0.0$. The segment of the modular coil determined by $c_{3}$ and $c_{4}$ lies along the toroidal direction, thereby partially replicating the effects of a hardcore.)

VACUUM MAGNETIC-FIELD PROFILES AND OTHER PROPERTIES OF THESE MODULAR STELLARATORS

The lisi set of discrete-coil modular stellarators examined with the aforementioned conirol suiface, coil-winding law and high-beta values for the shaping parameters had a coil-set ma or radius of eight and an individual coil radius of about one, as measured in the z-direction by $r_{b}{ }^{*}$ sin $C$ (implying a coil aspect ratio of eight). These stellarators had six field periods, six coils per field period, moderate lateral deformation, and a D-shaped control-surface cross section (Figs. 2a through 2e). (See Appendix $B$ for an alternate approach to evaluating the average radius of this control-surface and coil and the resultant aspect ratio.) The parameter search in the immediate neighborhood of the high-beta coefficients began with an investigation of the effect of tilting the plane of the coils through adjustments of $c_{5}$. Decreasing the tilt from veritical from positive 5 degrees to zero raised $V^{\prime}$ and lowered $<B>$ (Figs. 3a through 3d). A zero argree positlve tilt had the least magnetic hill, as evident in plots of $V$ ra: and

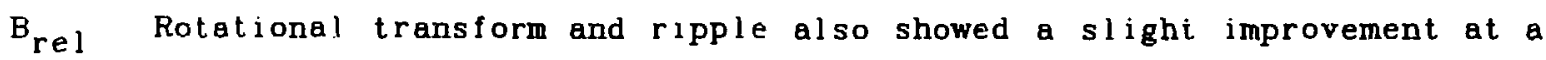
tilt of zero. Tilt steps of 2.5 degrees were too small to show much effect, so the range was exterded to 10 degrees in subsequent studies. In the presence of a small, uniform vertical magnetic field (2\% of the toroidal magnetic fleld), a 5-degrees positive tilt showed slightly less ragnetic hill than positive 10 degrees or zero tilt (Figs. 4a through 4d). A 5-degree positive tilt in the presence of this uniform vertical magnetic field produced the best overall results with respect to minimum magnetic hill, least ripple, and confinement of rotational transform between rational surfaces, which prevents magnetic islands (best observed and confirmed in puncture plots such as Fig. 5). Tilting in the negative direction led to large islands at the magnetic axis and a rotational-transform profile that iniersected the rational surface of $\iota=1 / 2$, independent of the presence of the small, uniform vertical 
magnetic field. In all the aforementioned cases, most of the rotational-transform profile had magnitude greater than one-half per. field perlod and had positive shear. No wells were found.

The effects $c^{\cdot}$ modifying the lateral deformation of a coil were investigated through adjustments of the shaping parameters, $c_{3}$ and $c_{4}$, while the tilt was held constant at 5 -degrees positive and the uniform magnetic field was turned on. As seen in Figs. 6a through 6d, increased lateral deformation accomplished with a $10 \%$ increase of $c_{4}$, enhanced the magnetic hill. reduced the magnitude of the rotational-transform profile to a point of intersecting the rational surface of $\iota=1 / 2$ (leading to a pair of extremely large magnetic islands), and increased the ripple. Aaditional increases of $c_{4}$ would have lowered the rotational-transform profile below this rational surface; the magnetic hill would have increased as well as the ripple and shear. On the other hand, a decrease in the laleral deformation iccomplished by decreasing $C_{4}$ by $10 \%$ increased the magnitude of the rotational-transform profile so that it intersected the less important, still significantly detrimental rational surface of $\iota=3 / 5$. Similar behavior was observed for $c_{3}$ but to a lesser extent than for $c_{4}$ (Figs. 7a through 7d). The best values for $c_{3}$ and $c_{4}$ in the discrete-coil vacuum case were within plus $5 \%$ and minus $10 \%$ of those suggested by Betancourt's high-beta, continuous-current-sheet models. 4 iniering the lateral deformation did not appear to be a feasible way to produce a vacuum magnetic well in the 36-coil stellarator configurations.

The next major step in the parameter search was to increase the number of coils per field period from 6 to 10 . This resulted in a proportionate reduction of the magnetic hill, as illustrated by $V^{\prime} r e l$, Brel, and ripple (Figs. 8a through 8d). The rotational-transform profile for the high-beta coefficlents was flatter and lower for 10 coils per field period than for 6 coils per fleld period, which led to a peculiar problem for no vertical magnetic field and zero tilt (not illustrated). The whole profile was so close to the rational surface of $\iota=1 / 2$ that large magnetic islands formed that dominated the plasma. Flux-surfaces could not form near the magnetic axis, only near the separatrix. If this flat, rotational-transform profile could be translated, intact, below the rational surface, it might be well confined between rational surfaces. A positive tilt of 5 degrees restored the flux surfaces to their normal concentric D-shapes. An additional 5-degrees positive tilt flattened the rotational transform profile further and pulled it 
below the detrimental rational surface of $\iota=3 / 5$, which the positive 5-degrees tilt profile intersected near the separatrix. Thus, regardless of the presence of the small, uniform vertical magnetic field, positive tilts of 5 to 10 degrees gave better radial magnetic-field profiles than negative or nonexistent tilts. The $2 \%$ vertical magnetic field increased the ripple, magnetic hill, and shear.

The effects of aspect ratio, spread of the conductor cross section, geometry of the control-surface cross section and cycloidal oscillation amplitude were investigated for the 10-coils-per-field-period modular stellarators in the presence of a small. uniform vertical magnetic field and a positive 5-degrees tilt (Figs. 9a through 9d). The coil aspect ratio was incremented in 5\% steps in both directions from the high-beta value by suitable adjustments of $r_{b}{ }^{*}$. An aspect-ratio increase enhanced the magnetic hill. A decrease diminished the hill and flattened the rotational-transform profile - both highly desirable qualities. The spread of the modular-coil conductor (ratio of toroidal width to radial thickness) was increased from an intermediate value of 0.4 , in regular steps, to 10 . In separate tests, the finite cross-section conductor was replaced by a filament. The overall effect of these two variations upon the magnetic field profile was less than $1 \%$. The rotational-transform profile was somewhat more sensitive to increases of spread, especially near the magneic axis; still the magnitude of the effect was less than $2 \%$.

The coefficient $C$ in the control-surface equation affects both height and minor axis of the control-surface cross section (Fig. 1). The specific direction of the change depends on its starting value relative to $\pi / 2$. Near the recommended high-beta magnetic well value of $C$ of 1.2 , an increase produced a small increase in height and a larger decrease in minor radius, accompanied by an increase in $\langle r\rangle$ and a decrease in aspect ratio. Increases of $\mathrm{C}$ greater than $10 \%$ i owered the rotational-transform profile to the point of intersecting the $\iota=1 / 2$ rational surface, which led to large magnetic islands. Similar magnitude decreases of $C$ elevated the rotational-transform profiles above this rational surface. This was accompanied by increased shear with the consequent intersection of the profile with the $\downarrow=3 / 5$ rational surface near the separatrix. (lncreases of $C$ beyond $\pi / 2$ reversed the height trend while continuing the reduction of the minor axis.) Adjustments of $c$ in 
the immediate neighborhood of the high-beta value did not improve the magnetic-field profile.

The concavity of the coil and of the control-surface cross section was alterable through $\Delta_{2}{ }^{*}$ (note: such changes will also affect $\langle r\rangle$ and, therefore, the aspect ratio). More concavity (reduced $\Delta_{2}{ }^{*}$ ) near the high-beta value of $\Delta_{2}{ }^{*}$ led to flux-surface problems: even a $2 \%$ reduction of $\Delta_{2}{ }^{*}$ forced the rotational-transform profile into the immediate neighborhood of the rational surface at $\iota=1 / 2$, which resulted in large magnetic islands. Less concavity (increased $\Delta_{2}$ ) was possible, but this aggravated the magnetic hill problem, raised the rotational transform, and detrimentally affected the shear.

The radius of gyration of the helical control-surface (sometimes called osculation amplitude, helical amplitude, or cycloidal-oscillation Einplitude) was increased by increasing $\Delta_{1}{ }^{7}$ with the consequence of the rotational-transform profile intersecting the rational surface of $\iota=1 / 2$ and. again. creating large magnetic islands. Decreasing the radius of gyration in small steps was possible, but it was accompanied by undesireable increases in magnetic hill.

The only improvement of the magnetic-field profile in the 10-coilsper-field-pericd study resulted from an increase in coil density, a decrease of aspect ratio, and a small positive tilt of the coils. Comparisons with the 6-coils-per-field-period findings indicate that nore coils per field period and a smaller aspect ratio will generally improve the magnetic-field profile.

During the preceding investigations a suspicion arose that increasing the number of coils per field period from 10 to 11 , even to odd, could reduce the ripple and perhaps improve the magnetic-field profile (Figs. 10a through 10d). This was tested with the discrete-coil code and not borne out; the improvement in magnetic-field profile appeared, on the other hand, to be the result of more coils. The rotational-transform profije flattened out further, but, again, this was due to a trend rather than a grouping according to odd-versus-ever number of coils. The tilt effects were also checked at 11 coils per field period at positive 5, 10 and 15 degrees. A positive 5-degrees tilt from vertical was best in all respects for the measured characteristics (Figs. 11 a through 11d).

Next, TORSIDO was applied to the modular-coil set from the most recently published continuous-current-sheet stellarator studies. ${ }^{4}$ This required a reduction of the major radius from 8 to 6 . The control-surface equation and 
coil-winding law were unchanged and the coefficients were set identical to the high-beta values. (Since the control-surface was nct changed, the $r_{b}{ }^{*}$ radius and average radius of the coil were also not changed. Consequently, the coil aspect ratio must decrease from 8 to 6.) These configurations had 6 coils per field period and 6 field periods. Although this model had a high-beta well in the current-sheet approximation, no well was found in the discrete-coil vacuum representation. As a possible remedy of this discrepancy, $M$. Mond suggested increasing the helical amplitude of the plasma to increase the curvature of the magnetic axis and reduce the magnetic hill, +t+ Subsequently, a parameter search of $\Delta_{1}{ }^{*}$ for aspect-ratio-six modular stellarators was undertaken. The plasma helical amplitude was modified by variations of the cycloidal oscillation amplitude of the coils (Figs. 12a through 12d). These tests proved that a nearly optimum helical amplitude (Curve \#3) had been recommended by the previous high-beta investigations from the standpoint of rotational transform, volume utilization, and minimum hill. No well was found by increasing the helical amplitude. In fact, the magnetic hill grew steeper as the helical amplitude increased and the plasma volume decreased. A $25 \%$ decrease of the magnitude of $\Delta_{1}{ }^{*}$ from the high-beta value (h.B.v.) caused a $6 \%$ increase in the radius of gyration and decreased the plasma volume by $15 \%$. This lowered the rotational transform without reaching the rational surface of $\iota=1 / 2$ and may have improved the magnetic-field profile slightly. An additional $25 \%$ decrease in $\Delta_{1}{ }^{*}$ increased the radius of gyration by approximately $10 \%$, lowered the rotational transform well below $t=1 / 2$, and drastically increased the shear. Positive shear of this magnitude for a plasma configuration with rotational transform below, but very near, the $\iota=$ $1 / 2$ rationel surface caused a significant deterioration of the flux surfaces near the separatrix and a substantial decrease of the effective plasma volume. To improve upon these configurations, the rotational-transform profiles must be flattened. The most significant trends resulting from increasing the helical amplitude of the plasma were an enhanced ripple and a suppressed rotational transform. Unfortunately, the shear was too large, thereby causing the plasma volume to be severely restricted.

\footnotetext{
Information provided by M. Mond, Consultant at Los Alamos. Department of Mechanical Engineering. Ben Gurion University, P.0.Box 653, Beer-Sheva 84105, I SRAEL .
} 
A further improvement of magnetic-field profiles over putlished recommendations was accomplished by increasing the number of coils per field period from 6 to 12 and then to 24, while keeping other parameters unchanged (Figs. 13a, 13b, and 14a through 14d). (1ncreasing the number of coils by an additional factor of 2 would have exceeded the memory limits of a CRAY 1-S using the present form of TORSIDO.) The spacing between coils in the 6-coils-per-field-period case was comparable to the radius of the coil; in the 24-coil case, the spacing was comparable to one-quarter of a coil radius, thereby approaching the current sheet condition. The magnetic hill diminished twofold when the number of colls was doubled the first time (to 12 coils per field period). The shape of the rotational-transform profile changed and its magnitude dropped. Ripple also halved but kept the same radial profile. Doubling the number of coils from 12 to 24 did not reduce the hill near the axis but did reduce it significantly near the separatrix, thereby changing the shape of the magnetic-field profile. The next doubling of the number of field coils would probably cause a further departure from a simple parabolic field profile, possibly evolving into a complicated profile with saddle points similar to that described by McNamara. ${ }^{1}$ The rotational-transform profile continued to evolve toward lower values while its minimum moved radially outward. Most of the reduction in ripple occurred near the separatrix. Clearly, it is highly desirable with respect to all measured characteristics to have at least 12 coils per field period in the present geometry.

\section{CONCLUSIONS}

The present work indicates that the discrete coils ar 2 responsible for the vacuum magnetic hills in the configurations under investigation. Therefore, we must tentatively conclude that the proposed modular-stellarator configuration will not have a magnetic well in a vacuum and thus will be unacceptable for start-up.

In view of the results of theoretical investigations of the plasma effects on magnetic wells ${ }^{2}$ the present computations indicate that the (finite beta) magnetic wells found for this particular modular stellarator 4 hinge on a continuous-sheet-current representation of the external coils, rather than on the effects of plasma; this conjecture is supported by the presently found linear scaling of the magnetic hill with separation between coils. 
A recent investigation of Heliacs by Shestakov and Mirin supports the first part of this conjecture with evidence of the detrimental effect upon vacuum magnetic wells of replacing a continuous-current sheet with modular coils. ${ }^{5}$ They found vacuum magnetic wells for several Heliac configurations, when their magnetic field was represented by skin currents flowing alon: equipotential contours of a magnetic surface in an asymmetric taraida? domain. Discretization into coils preserved the favorable low-shear rotational transform and small variation of $/ \mathrm{dl} / \mathrm{B}$ but not the well. The present investigation indicates that an increase in the number of coils per field period diminishes, but does not eliminate, the vacum magnetic hill. If the possibility of the existence of a successful start-up configuration Jor this particular sodular-stellarator can be judged by the diminishment of vacuum magnetic hills, it will require at least 12 coils per period. Furthermore, it will probably require a small positive $t i l t$ of the coils from vertical, the presence of a uniform vertical magnetic field, and judicious choices of coil deformation and helical amplitude such that the rotational transform lie well confined between the rational values of one-half and one-third.

No improvement in magnetic-field profile was achieved by decreasing the aspect ratio from 8 to 6 , and then to 4 as suggested by Bathke's invesigations ${ }^{3}$ (Figs. 15a through 15e). Even a microscopic investigation of the immediate neighborhood of the magnetic axis for $A=4$ yielded no well, although the hill was extremely flat. Generally, the magnetic hill, the magnitude of the rotational transform, and the ripple increased with decreasing aspect ratio. Thus far, in the present configuration we have no evidence to recommend one particular aspect ratio as more desirable than any athers tested in this study.

\section{DIRECTIONS FOR ADDITIONAL RESEARCH}

An interesting computational question remains unanswered regarding the persistence of the magnetic wells to the limit of small beta in the contiuous-current-sheet representations. This can be best answered by reducing beta in runs of the BETA code 4 with the same control-surface-configuration coefficients that produced the magnetic wells. Also of interest to the present investigation would be the determination of 
the effect of ripple (toroidal and poloidal) on the quality of the flux-surfaces.

A question of technique is whether combinations of parameter changes could produce a magnetic well in the neighborhood of the high-beta values where single parameter variations had not. This question was addressed during the $A=8$ runs by incrementing $C$ and $\Delta_{2}{ }^{*}$ in a constant ratio over the range 1.2 to 1.4 and 0.70 to 0.67 , respectively. It was hoped that such variations would reduce the agnetic hill and prevent magnetic islands by forcing the rotational-transform profile to be adequately flat and lie between the one-half and the one-third rational surfaces. Good flux surfaces were found (Figs. 16a through 16d). The puncture plots representing the nested magnetic-flux surfaces had more concave contours, just as the control-surface cross sections were more kidney-been-shaped than in previous trials. The initial consequences of adiustments from the recommended finite-beta values were flat rotational-transtorm profiles, which were well confined between the one- H: and cne-third rational surfaces, and significantly intiproved ripple bui no well. At the end of these adjustments, the area of the ccatrol-surface cross section changed by only $1 \%$; an increase of height was compensated by a decrease in width such that the surface and coil aspect ratios computed from the average-radius method remained unchanged. The ripple continued to decrease. The shear became more negative. And the plasma volume was drastically reduced by the new geometry and the plasma aspect ratio was, at least. doubled. Such investigations showed that though small increases of $C$ alone led to simple increases in height, combinations of decreases in $\Delta_{2}{ }^{*}$ and increases in $\mathrm{C}$ beyond $\pi / 2$ produced more concave cross sections. However, more concavity does not guarantee the production of magnetic wells. Furthermore, although single parameter searches are probably limited to finding improvements in magnetic profiles in the immediate vicinity of a particular set of coil and control surface parameters, other parameter domains with acceptable flux surfaces could be accessible through combined adjustments of coil and surface parameters. The goal of additional trials of multiple-parameter searches would be to force flux-suriace cross sections to take shapes like those that have been associated with mognetic welis. 
ACKNOWLEDGMENTS

I would like to thank C. G. Bathke for invaluable advice and assistance with computational matters relating to modifications of TURSIDO. I would also like to thank $O$. Betancourt for suggestions of starting parameters in this study and helpful discussions during the project. Also J.P. Mondt and A. Sgro provided many helpful discussions, encouragement, and valuable criticism of this document. A nagging concers since the conception of this project has been whether plasma introduced into a vacuun magnetic field with no magnetic well, but at least a flat magnetic profile, could dig its own well. R. A. Gerwin and A. H. Glasser brought the frequently-qucted, critical plasma-weli-digging reference to my attention and aided with its interpretation. 
1. B. MoNamara, K. Whiteman, J. Taylor, "Helical Fields Possessing Mean Magnetic Wells," in Plasma Physics and Controlled Nuclear Fusion Research (IAEA, Vienna 1965), Vol. 1, pp. 145-167.

2. J. M. Greene. J. L. Johnson, "Interchange Instabilities in Ideal Hydromagnetic Theory," in Plasma Physics Vol, 10, pp. 729-745 (1968).

3. R. Miller, C. Bathke, R. Krakowski, et al., "The Modular Stellarator Reactor: A Fusion Power Plant," Los Alamos National Laboraiory report LA-9737-MS (July 1983).

4. F. Bauer, O. Betancourt, P. Garabedian, Magnetohydrodynamic Equilibrium and Stabiiity of Stellarators (Springer-Verlag. New York, 1984).

5. A. 1. Shestakov, A. A. Mirin, "Design of Heliac Vacuum Magnetic Fields," UCRL-91870 Preprint (1984). 
APPENDIX A: Two Methods of Measuring Magnetic Well Depth

In a recent article $e^{2}$ well depth was measured in terms of $\langle B\rangle^{2}$ (instead of $\left.V^{\prime}\right)$. evaluated from $\int B d l / \int d l / B$, i.e., using the magnitude of the magnetic fieid on a flux surface integrated aiong a field-line trajectory. This measure gives the correct geometric perspective, but approximately doubles (for small hills and wells) the magnitude and sensitivity of the depth measure compared to $\langle B\rangle$. This method of computing magnetic-fleld profiles from $\langle B\rangle^{2}$ also has the advantage of removing the considerable influence of ripple on $d l$ in discrete-coil modeling by normalizing with respect to dl. The conventiona] method of measuring magnetic-field profile with the divergence of plasma volume with respect to flux, $\left(V^{\prime} \equiv \int d l / B\right)$, has the opposite radial behavior to $\langle B\rangle^{2}$, decreasing as one moves away from the magnetic axis for a well. This problem of perspective can be correcter by looking at

$$
\mathrm{V}_{\text {rel }} \equiv 1 \cdot 0-\left(\mathrm{V} \cdot / \mathrm{V}^{\prime}{ }_{0}\right)
$$

instead of $V^{\prime}$, where $V^{\prime}{ }_{0}$ is $V^{\prime}$ on axis. The adu se and potentiaily domincting effect of ripple on evaluation of $V^{\prime}$ for discreie coils can be eliminated by normalizing $V$ ' with respect to $\int d l$, which has been consistently apflied to all data and graphs displayed in this report. We have also computed $\mathrm{B}_{\text {rel }}$ (type 1) and $\mathrm{B}_{\text {rel }}$ (type 2) for comparison with $\mathrm{V}_{\text {'rel }}$, i.e.,

$$
\begin{aligned}
& \mathrm{B}_{\text {rel }}(\text { type } 1) \equiv\left(\left(\int \mathrm{dl} / \int \mathrm{dl} / \mathrm{B}\right) /\left(\int \mathrm{dl} / \int \mathrm{dl} / \mathrm{B}\right)_{0}\right)-1.0 \text { and } \\
& \mathrm{B}_{\text {rel }}(\text { type } 2) \equiv\left(\left(\langle\mathrm{B}\rangle^{2}\right)^{1 / 2} /\left(\left\langle\mathrm{B}_{0}\right\rangle^{2}\right)^{1 / 2}\right)-1.0
\end{aligned}
$$

and find them all nearly identical. Here $\left\langle B_{0} s^{2}\right.$ and $\left(/ d l / \int d l / B\right)_{0}$ by definition are evaluated on axis. 
APPENDIX B: Average Radius of Control Surface and Resultant Coil Aspect Ratio

The average radius of the control-surface upon which the coil is laid could be estimated from the radius of a circle with area equal to that of an ellipse with the same major and minor axes as the D-shaped cross section:

$$
\langle\mathrm{r}\rangle=\left(\Delta_{2}^{*} \sin C\right)^{0.5} \mathrm{r}_{\mathrm{b}}^{*}
$$

Even if the control-surface is concave, that is, not flat on the inner side, the area of the cross section can be represented to a close approximation by the differences of half ellipses, and the same equation for the average radius applies. Thus for Betancourt's suggested values for control-surface parameters, the average coil radius is 0.88 instead of 1.0 , and the corresponding coil aspect ratio $\mathrm{a} .1$ for a major radius of 8 . (The smaller coil aspect ratio of 8 was based upon the $r_{b}{ }^{*}$ sin $C$ value for the coil radius .)

The largest plasma volume observed had minor and major radii less than half the rodii of the coil. Sonsequently, for the above example with coil radius 0.88 , the plasma aspect ratio must be at least 18 or more, depending on the limitations imposed by ratiunal surfaces. 


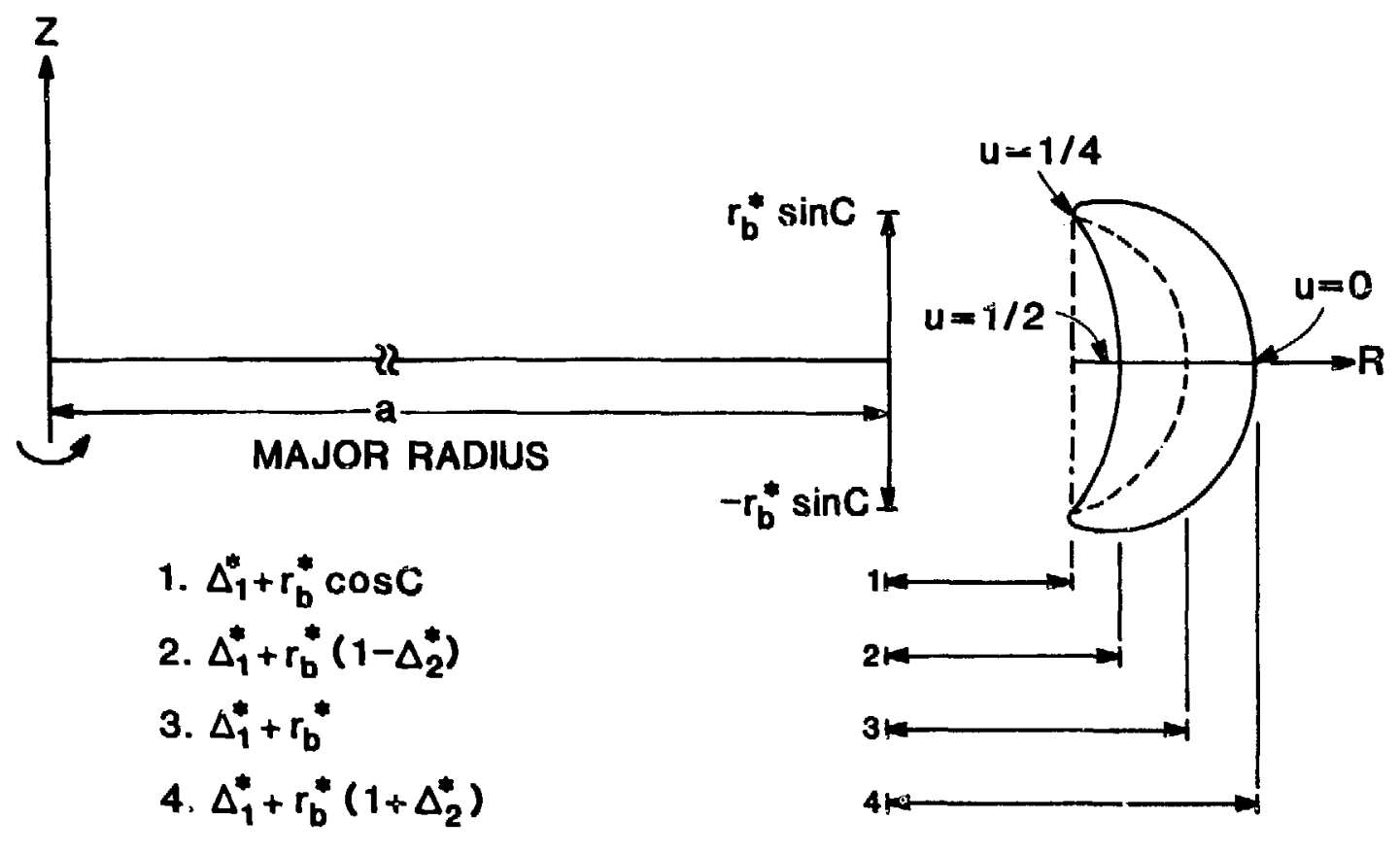

Fig. 1. Control Surface Cross-Section.

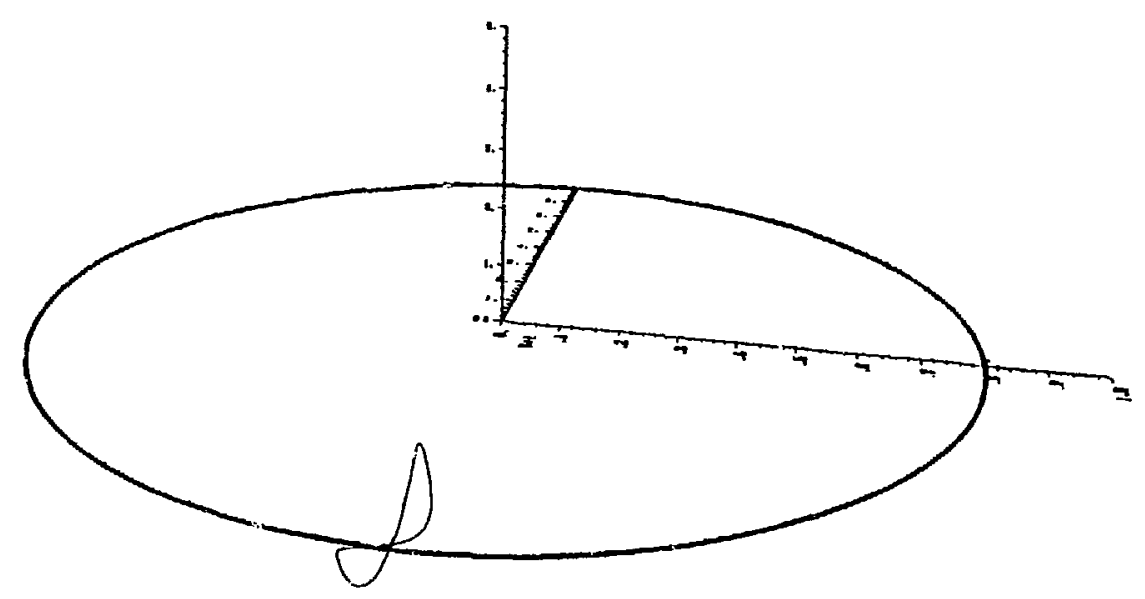

Fig. 2a. Line Representation of Single Coil (major radius included for reference).

Major radius (a): 8 Coils per field period: 6 Coil aspect ratio (A): 8 Coil-Winding Law and Control-Surface Equation Coefficients: High-Beta Values 
APPENDIX B: Average Radius of Controi Surface and Resultant Coil Aspect Ratio

The average radius of the control-surface upon which the coil is laid could be estimated from the racius of a circle with area equal to that of an ellipse with the same major and minor axes as the D-shaped cross section:

$$
\left\langle r>=\left(\Delta_{2}^{*} \sin c\right)^{i .5} r_{b}^{*}\right.
$$

Even if the control-surface is concave, that is, not flat on the inner side, the area of the cross section can be represented to a close approximation by the differences of half ellipses, and the same equation for the average radius applies. Thus for Betancouri's suggested volues for control-surface parameters, the average coil radius is 0.88 instead of 1.0 , and the corresponding coil aspect ratio 8.1 for a major radius of 8 . (The smaller coil aspect ratio of 8 was based upon the $r_{b}^{*} \sin c$ value for the coil radius.)

The largest plasma volume observed had minor and major radij less than half the radii of the coil. Consequently, for the above example with coil radius 0.88 , the plasma aspect ratio must be at least 18 or more, depending on the limitations imposed by rational surfaces. 


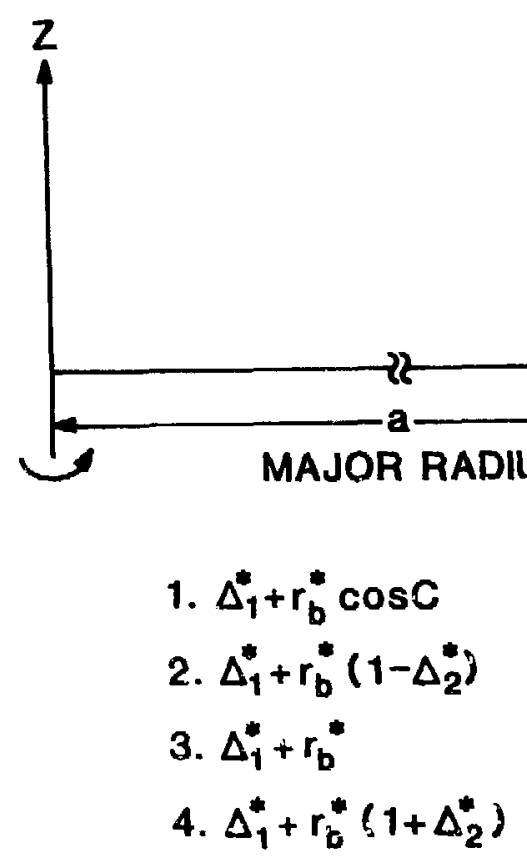

Fig. 1. Control Surface Cross-Section.

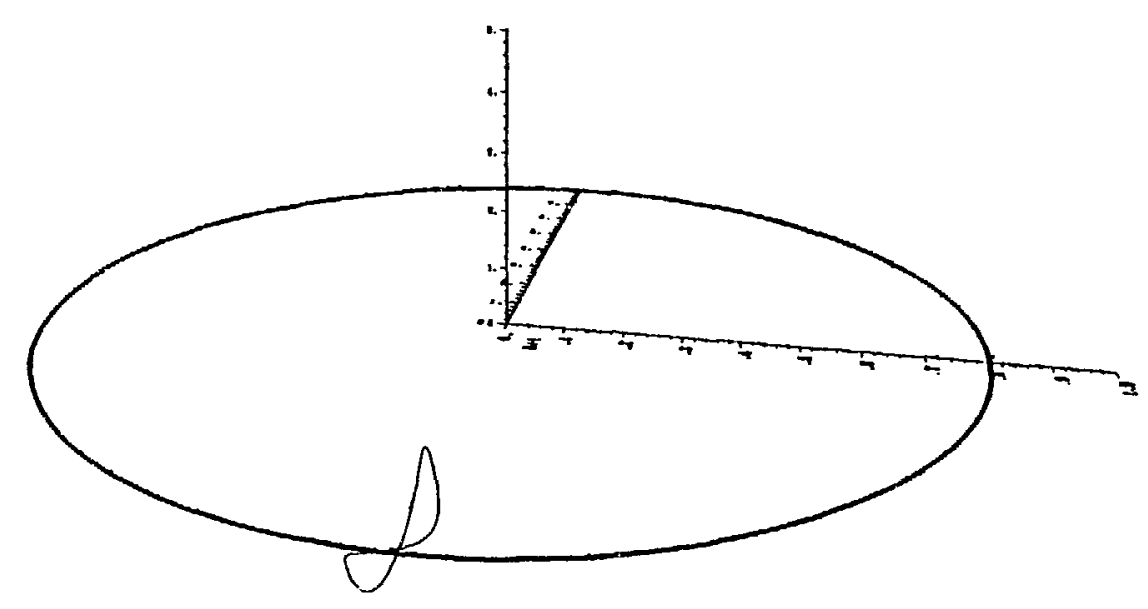

Fig. 2a. Line Representation of Single Coil (major radius included for reference)

Major radius (a): $8 \quad$ Fiela periods (Q) : 6

Coils per field period : 6 Coil aspect ratio (A) : 8

Coil-winding Law and Control-Surface Equation Coefficients: High-Beta Values 


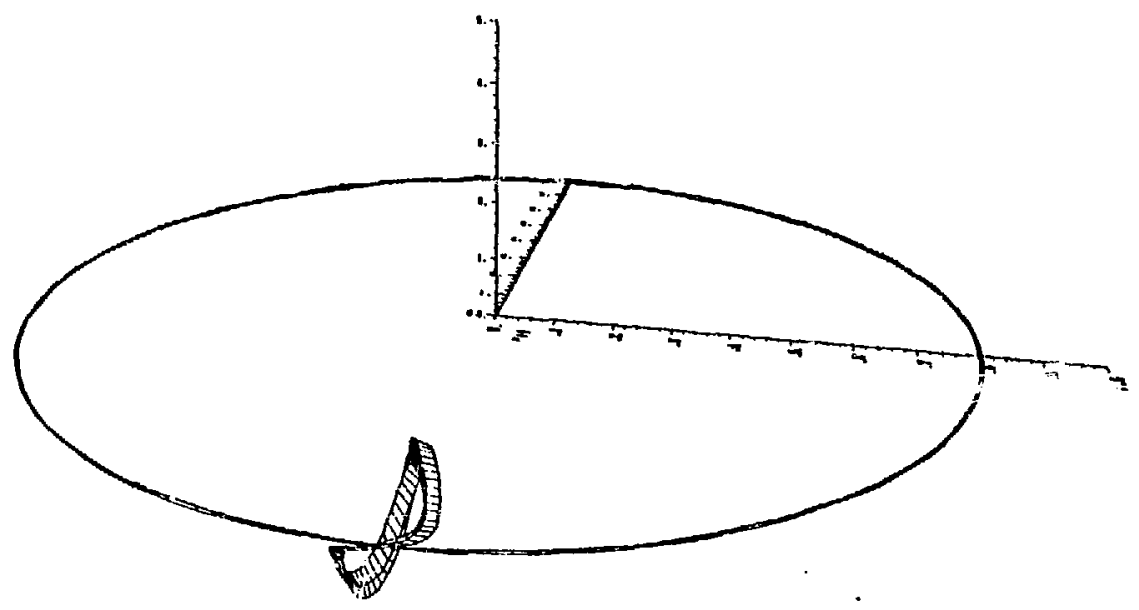

Fig. 2b. Polygon Representation of Single Coil (oiherwise, same as Fig. 2a).

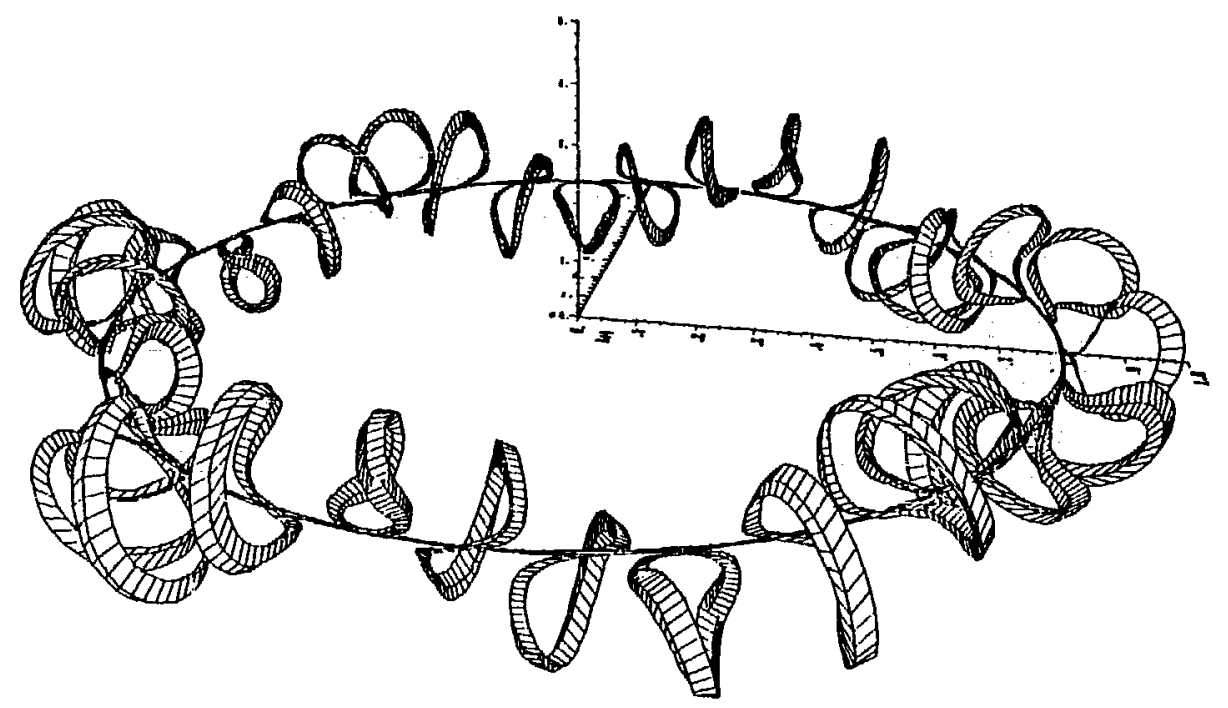

Fig. 2c. Polygon Representation of 36-Coil Set (otherwise, same as Fig. 2a). 


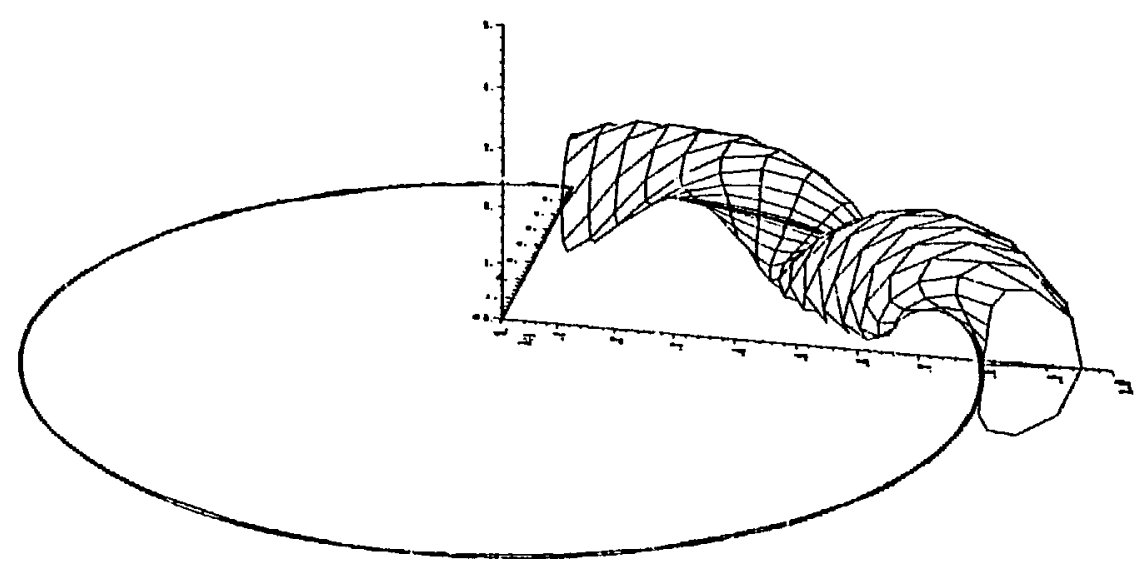

Fig. 2d. One-fourth of Control Surface and D-Shaped Cross Section (otherwise, same as Fig. 2a).

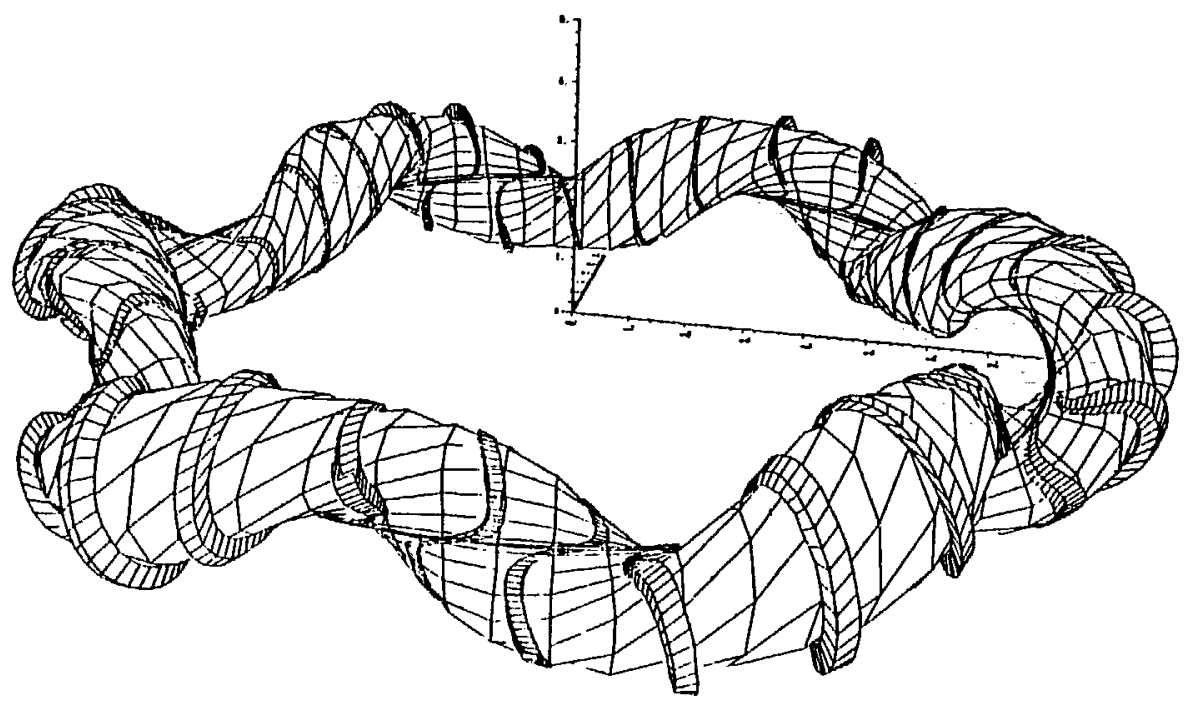

Fig. 2e. Entire Control Surface and Modular Coil Set (otherwise, same as Fig. 2a). 


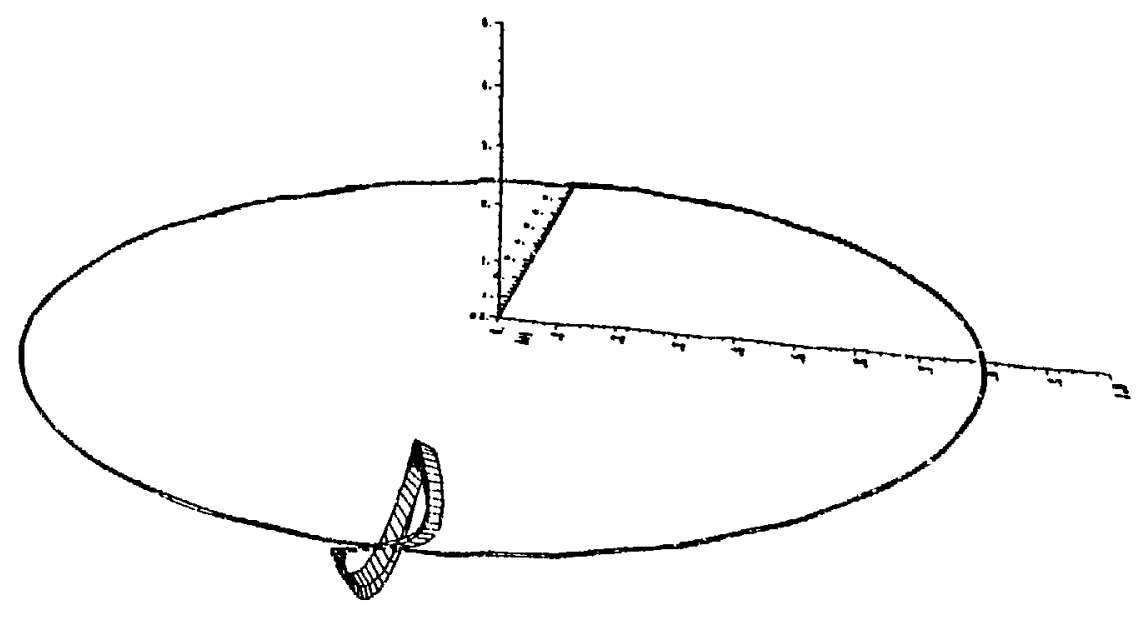

Fig. 2b. Polygon Representation oi Single Coil (otherwise, same as Fig. 2a).

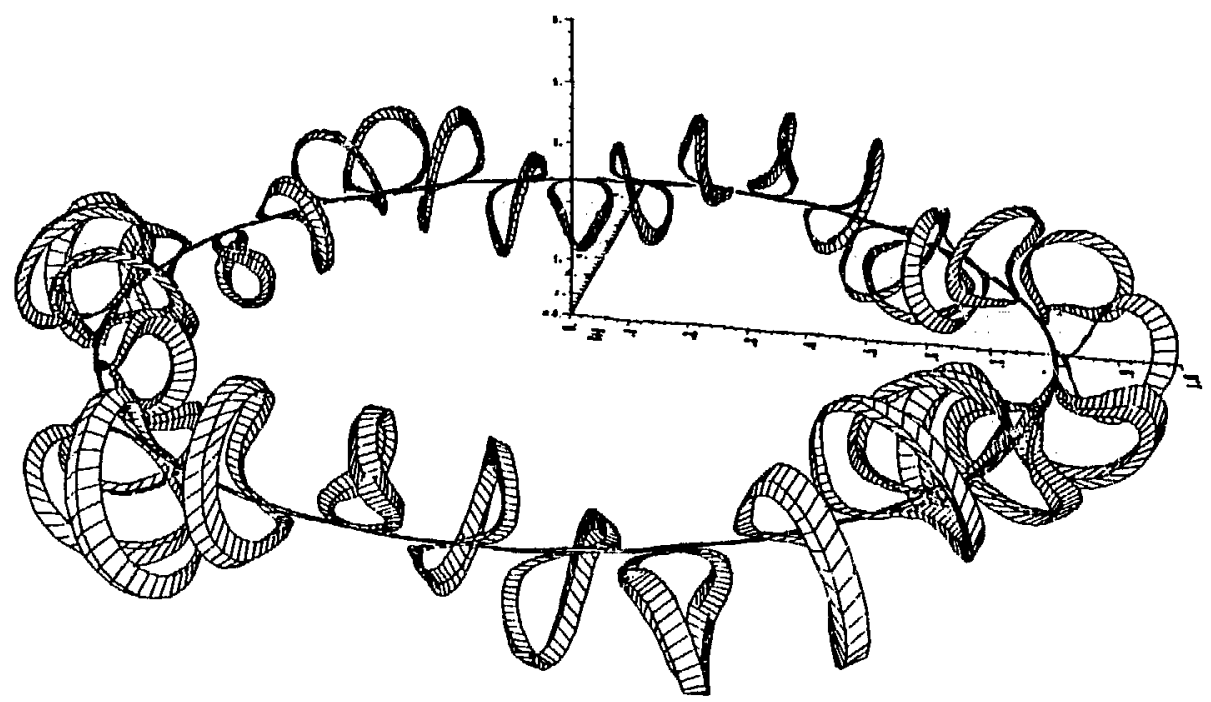

Fig. 2c. Polygon Representation of 36-Coil Set (otherwise, same as Fig. 2a). 


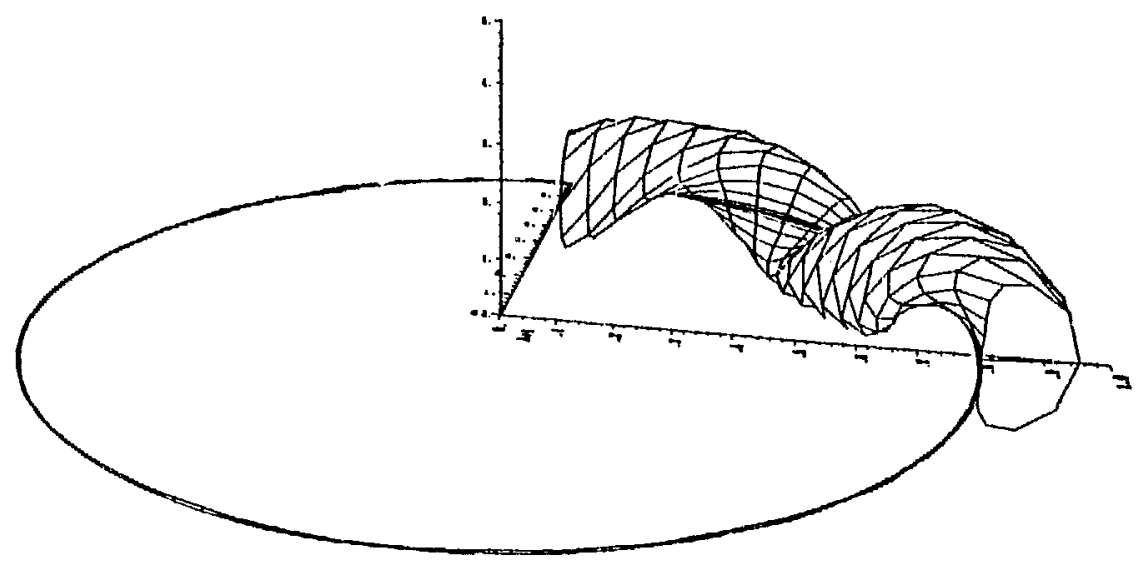

Fig. 2d. One-fourth of Conirol Surface and D-Shaped Cross Sectjon (otherwise, same as Fig. 2a).

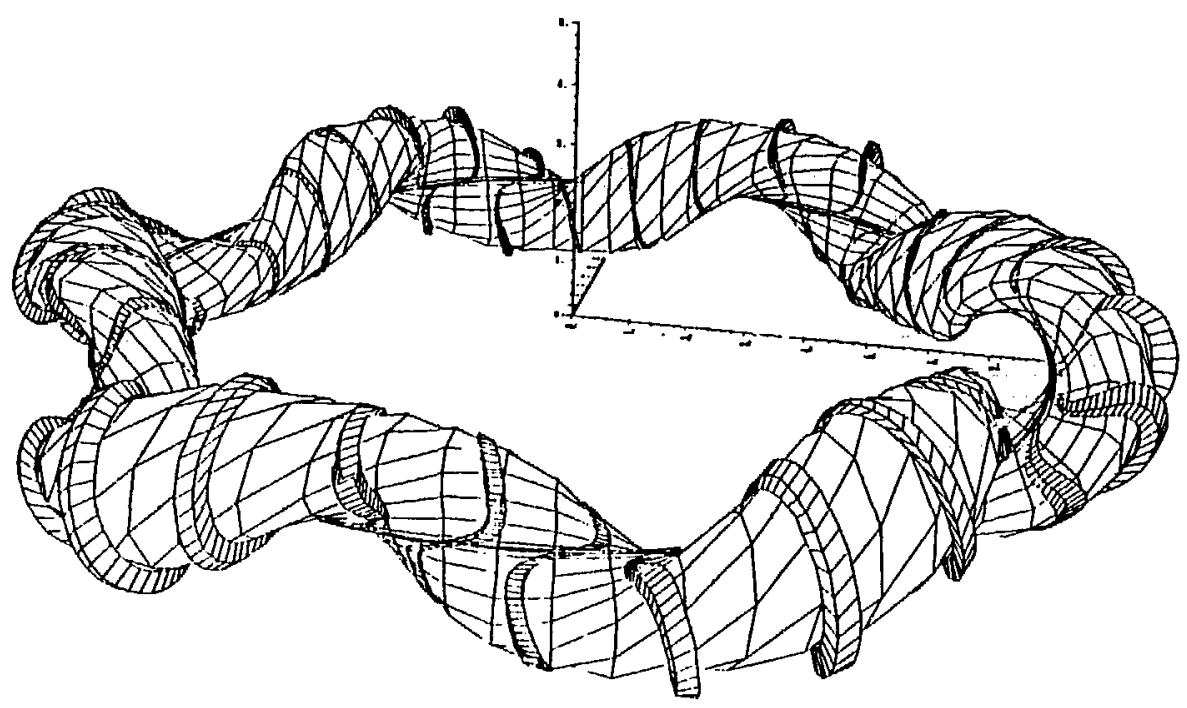

Fig. 2e. Entire Control Surface and Modular Coil Set (otherwise, same as Fig. 2a). 

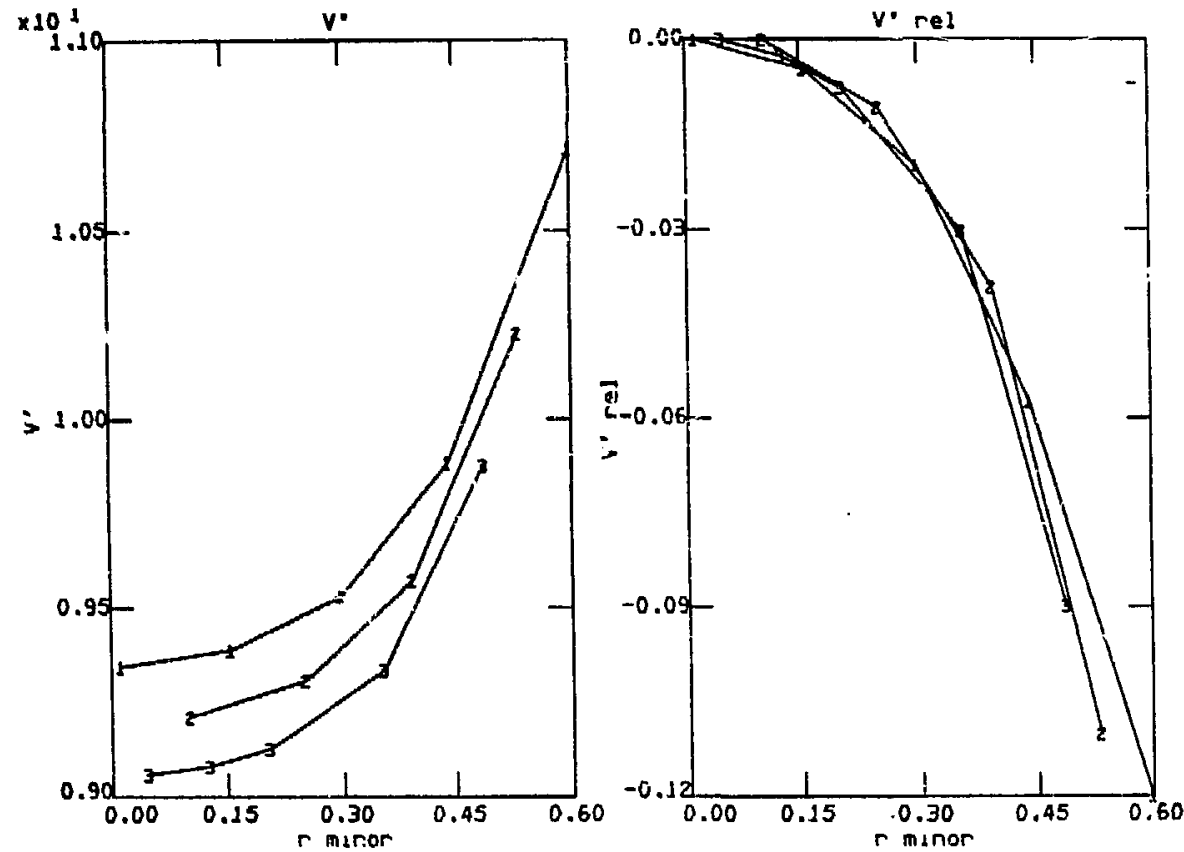

Fig. 3a. $V$ and $V^{\prime}$ rel: The Effects of Coil Tilt on The Magnetic Profile, Rotational Transform and Ripple (no E vertical). Curve \#1 - plus 5 degrees tilt from vertical, Curve \#2 - plus 2.5 degrees tilt, and Curve \#3 - zero degrees tilt.
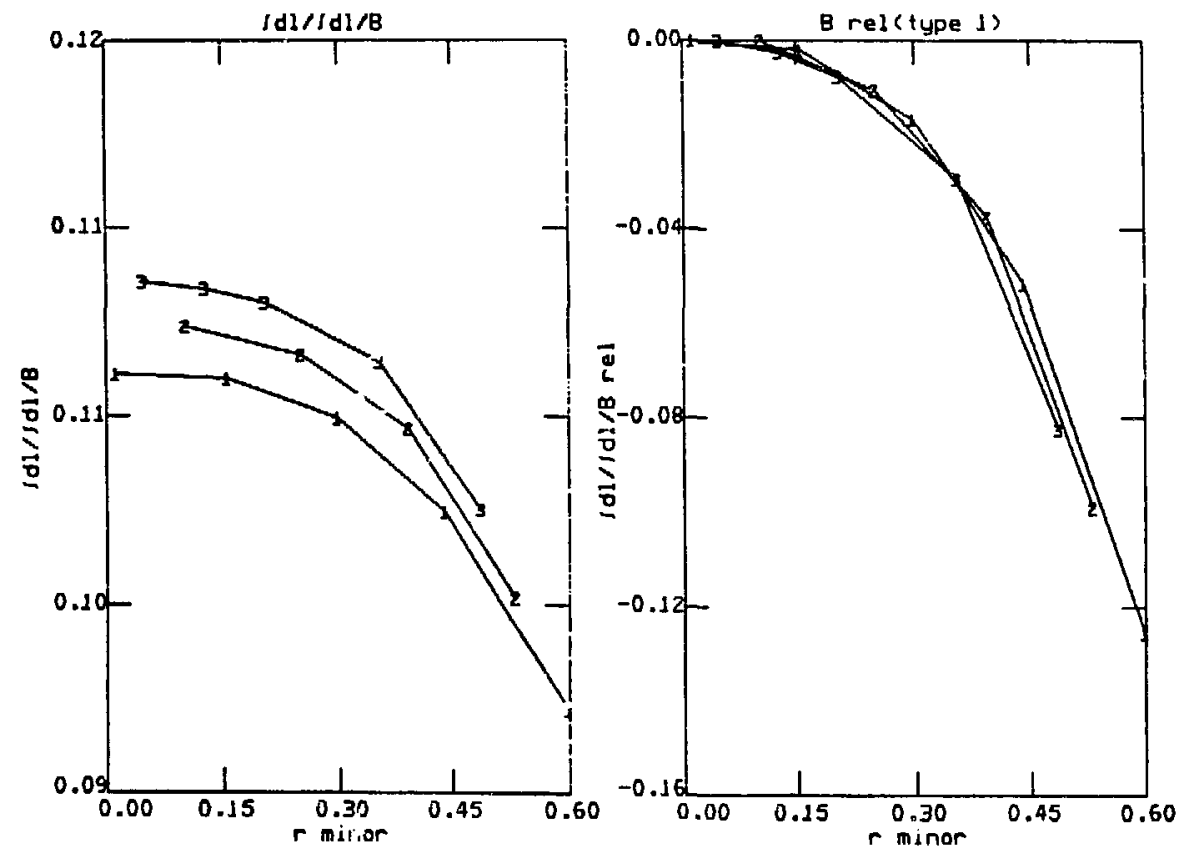

Fig. 3b. $<B>$ and $B_{\text {rel }}$ (type 1) (otherwise, same as Fig. 3a). 

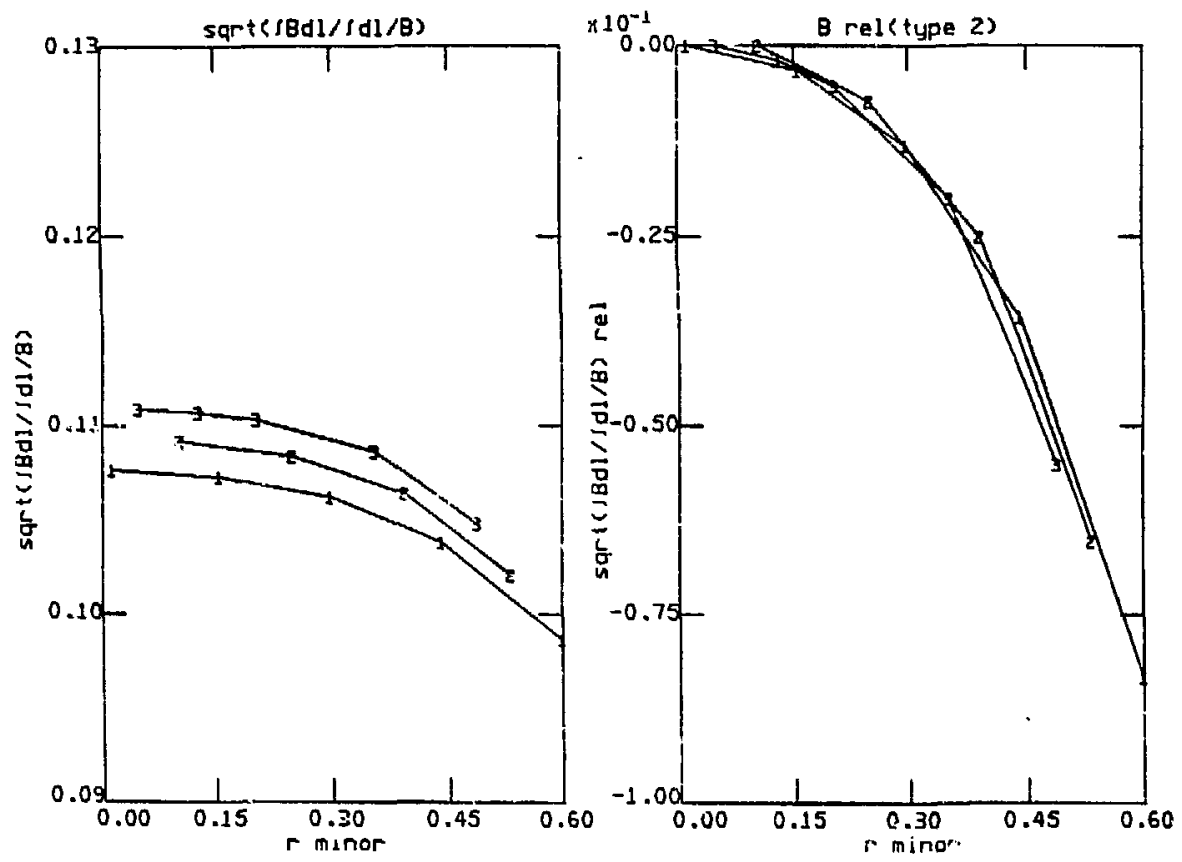

Fig. 3c. <B> and $B_{\text {rel }}$ (type 2 ) (otherwise, same as Fig. 3a).
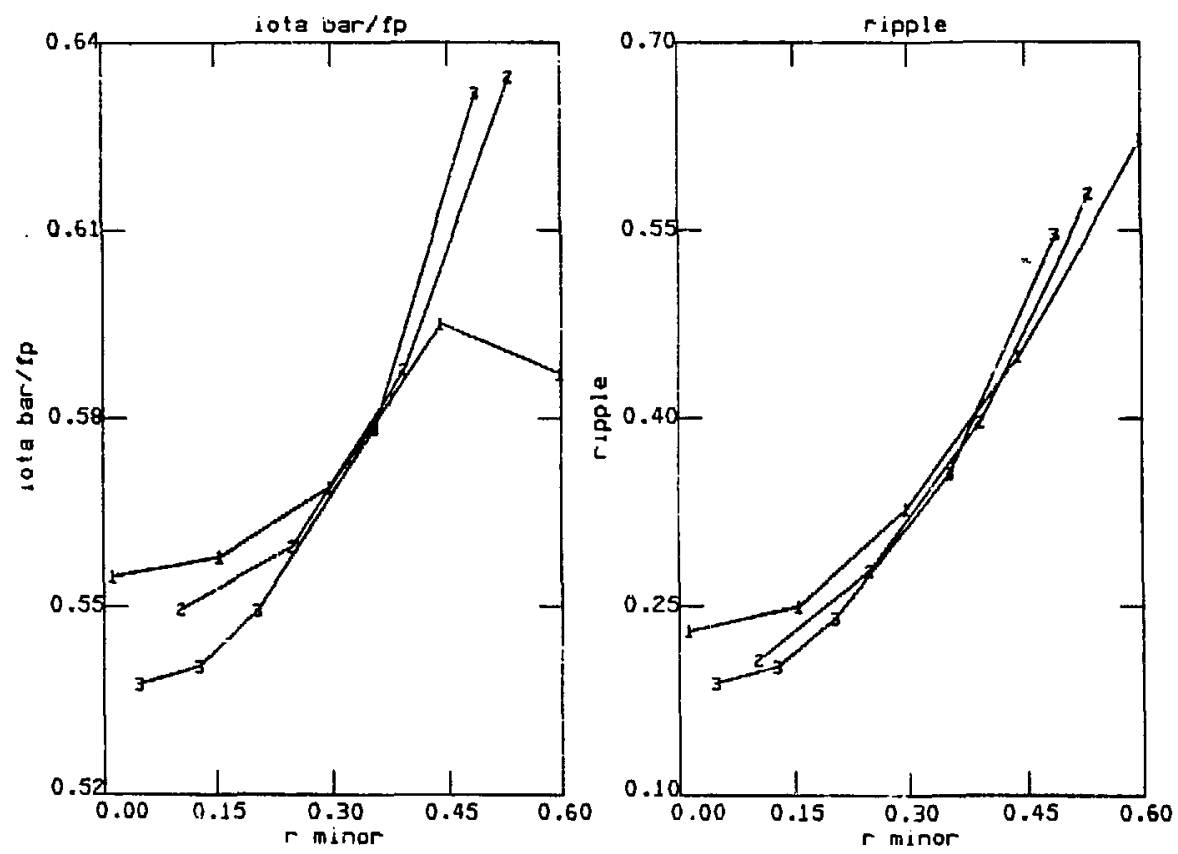

Fig. 3d. lota Bar per Field Period and Rippia (otherwise, same as Fig. 3a). 

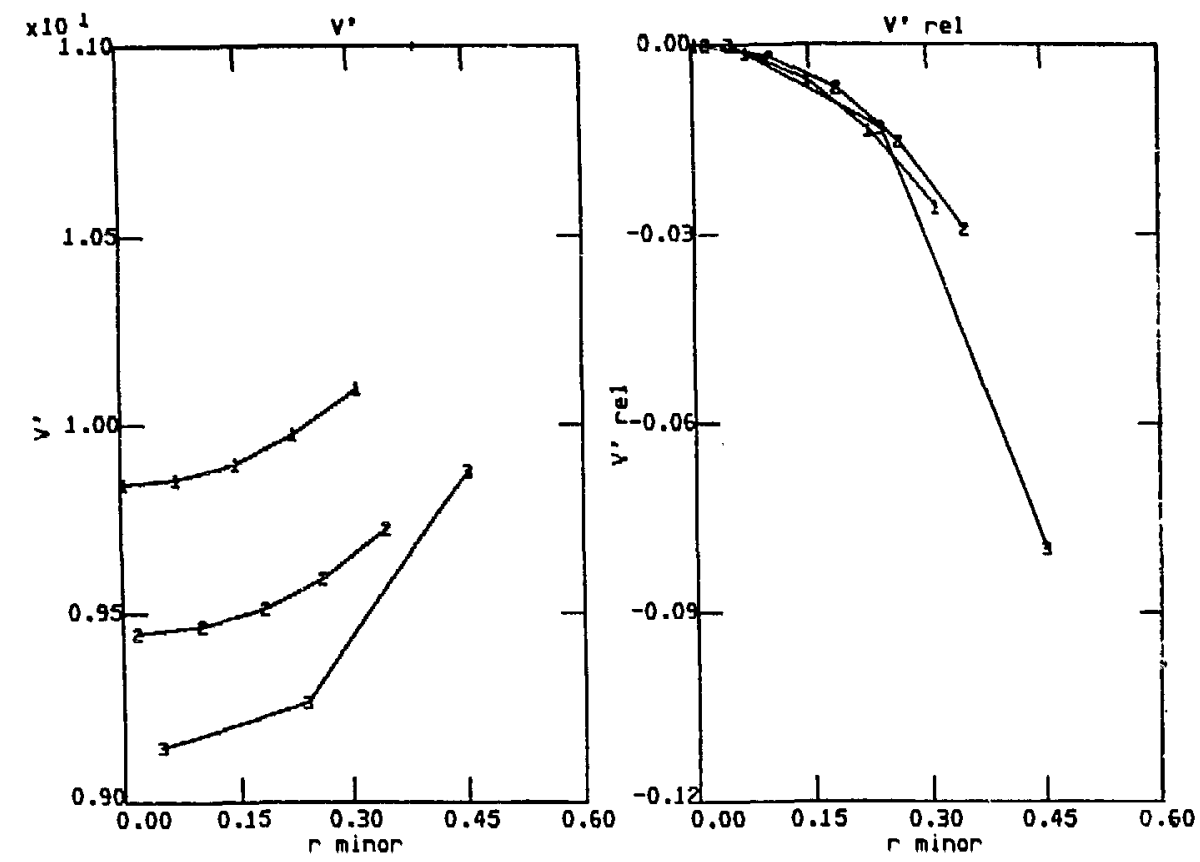

Fig. 4a. $V^{\prime}$ and $V^{\prime}$ rel: The Effects of Coil Tilt on The Magnetic Profile, Rotational Transform, and Ripple $\left(B_{\text {vert }}=2 \% B_{\text {tor }}\right.$

Curve \#1 - plus 10 degrees tilt from vertical, Curve $\# 2$ - plus 5 degrees tilt, and Curve \#3 - zero degrees tilt.
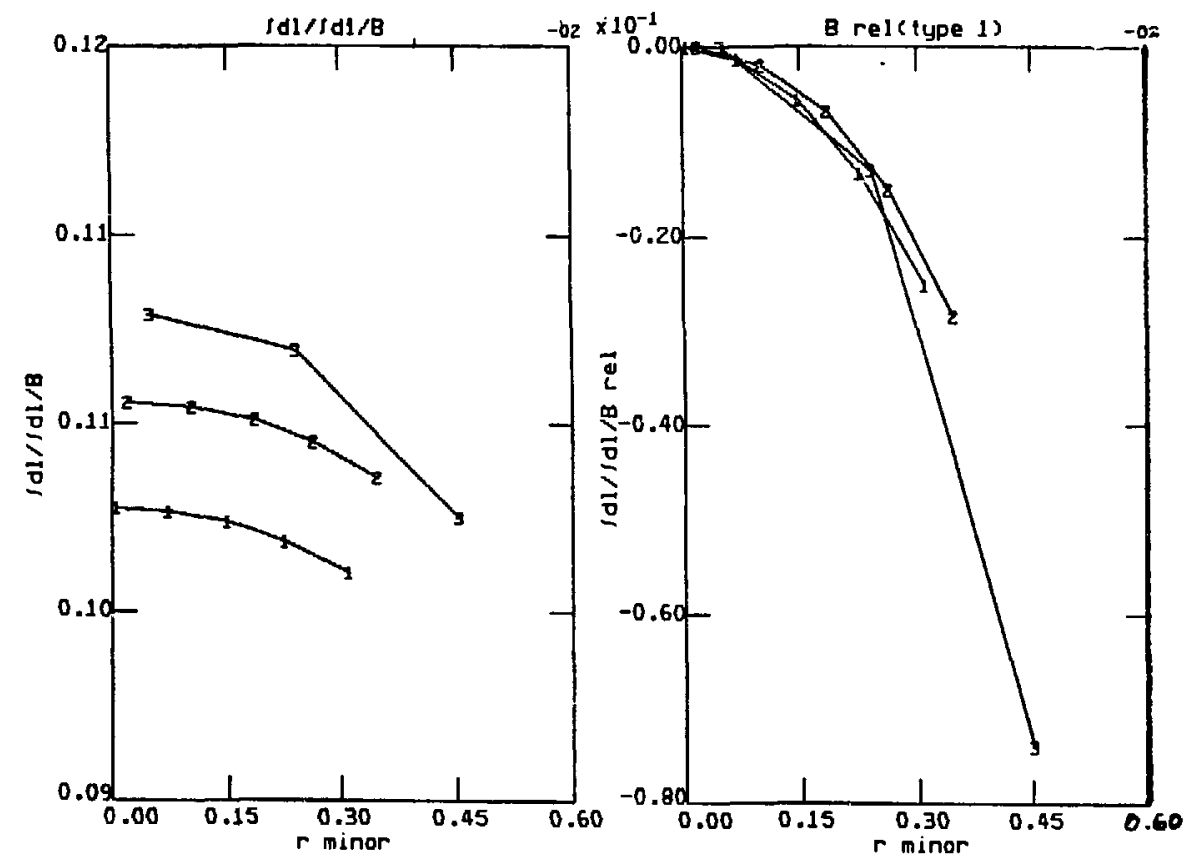

Fig. 4b. $<B>$ and $B_{\text {rel }}$ (type 1) (otherwise, same as Fig. 4a). 

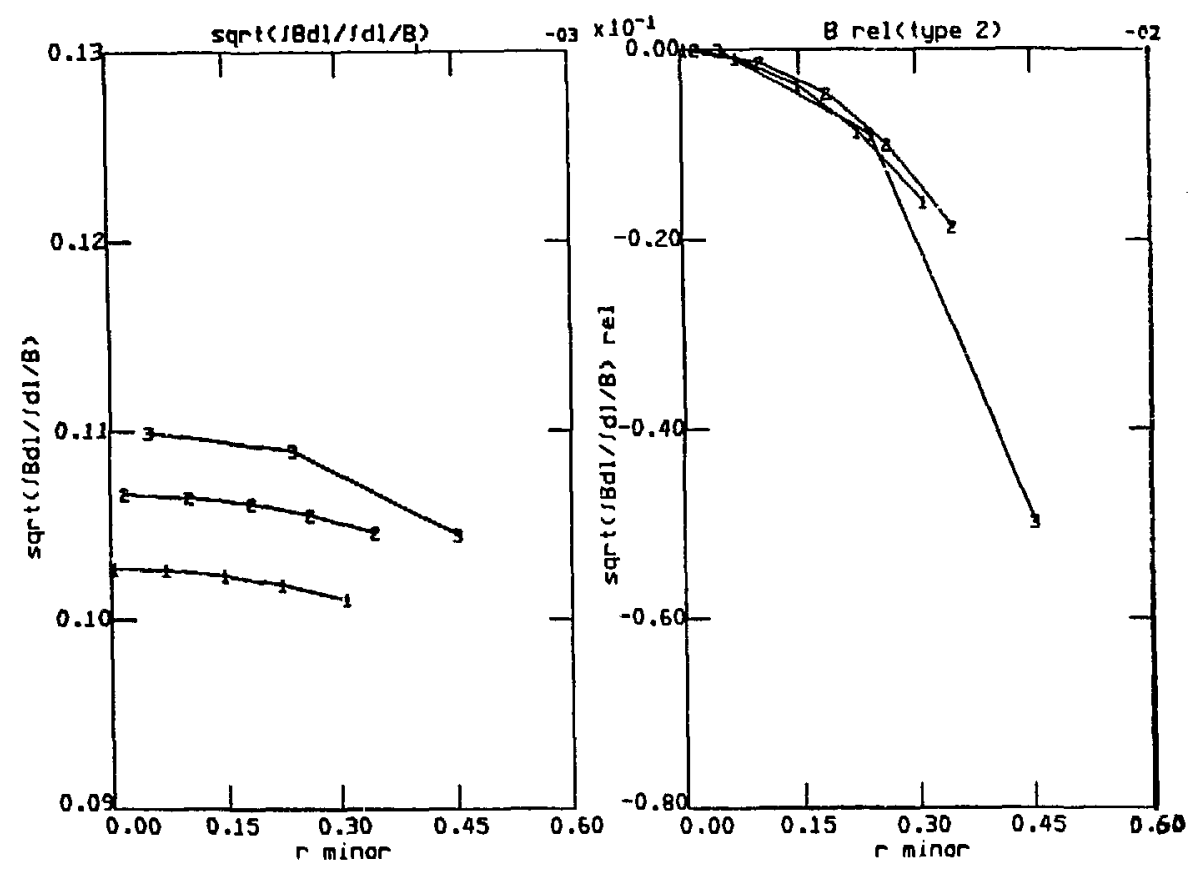

Fig. 4c. $\left\langle B>\right.$ and $B_{\text {rel }}$ (type 2) (otherwise, same as Fig. 4a).
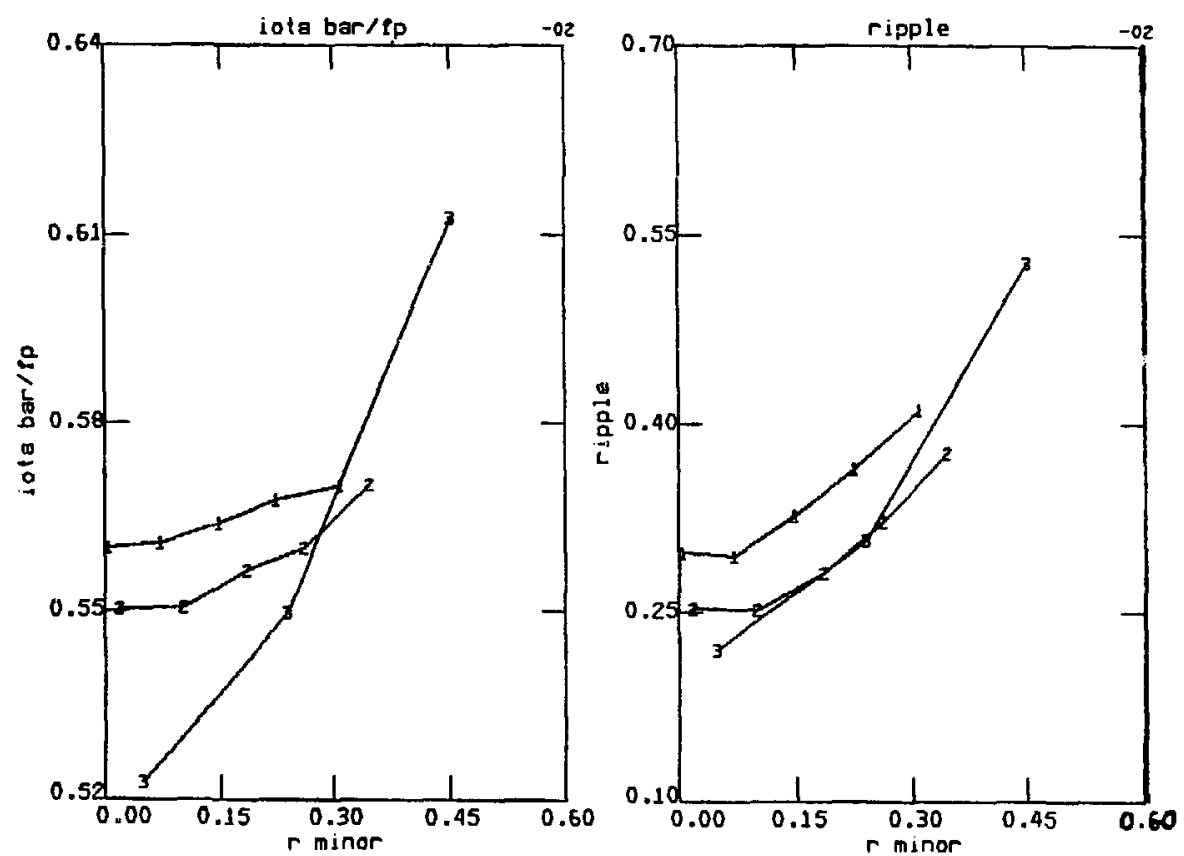

Fig. 4d. Iota Bar per Field Period and Ripple (otherwise, same as Fig. 4a). 

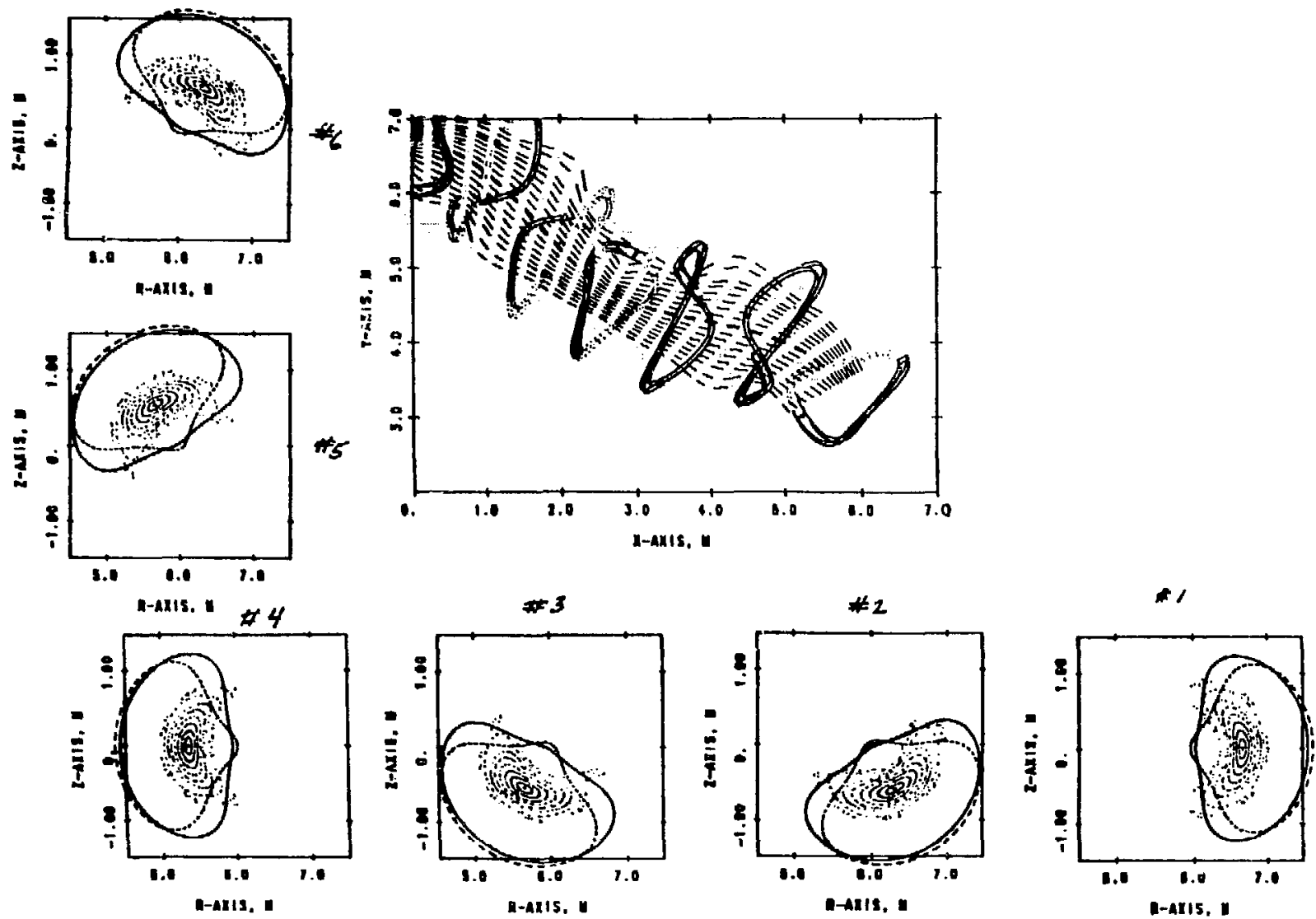

Fig. 5. Puncture Plots and Top-down View of Coils and Flux Surfaces.

Major radius (a): 8

Colls per fleld period: 6

B Vertıcal: $2 \%$ of $B$ toroidal
Field periods (Q): 6

Coil aspect ratio $(A): 8$ 



Fig. 6a. $\quad V^{\prime}$ and $V^{\prime}$ rel: The Effects of $c_{4}$ Lateral Deformation on Magnetic Profile, Rotational Transform and Ripple (2\% B vertical). Curve \#2- $c_{4}$ at nominal value of 0.625 , Curve \#1 - $0.9 \times$ nominal, and Curve \#3 - 1.1 x nominal.
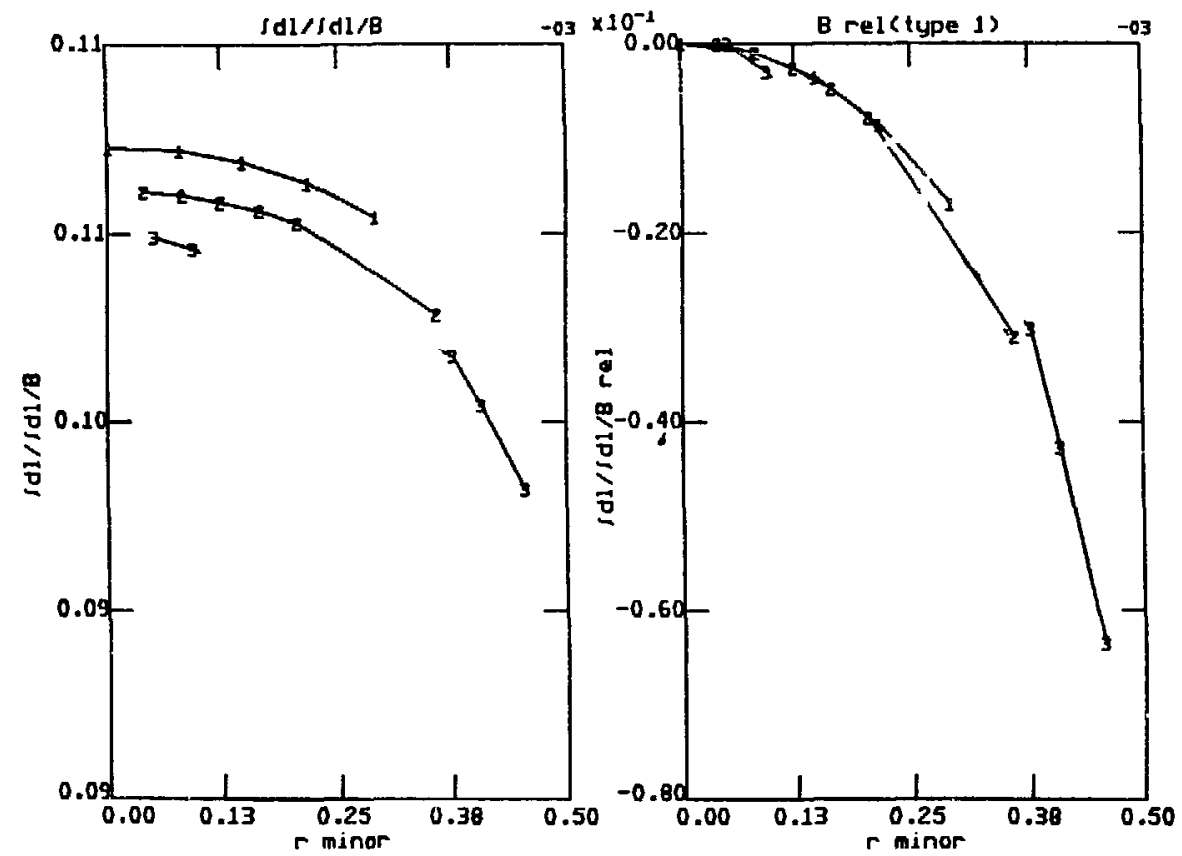

Fig. 6b. $<\mathrm{B}>$ and $\mathrm{B}_{\text {rel }}$ (type 1) (otherwise, same as Fig. 6a). 


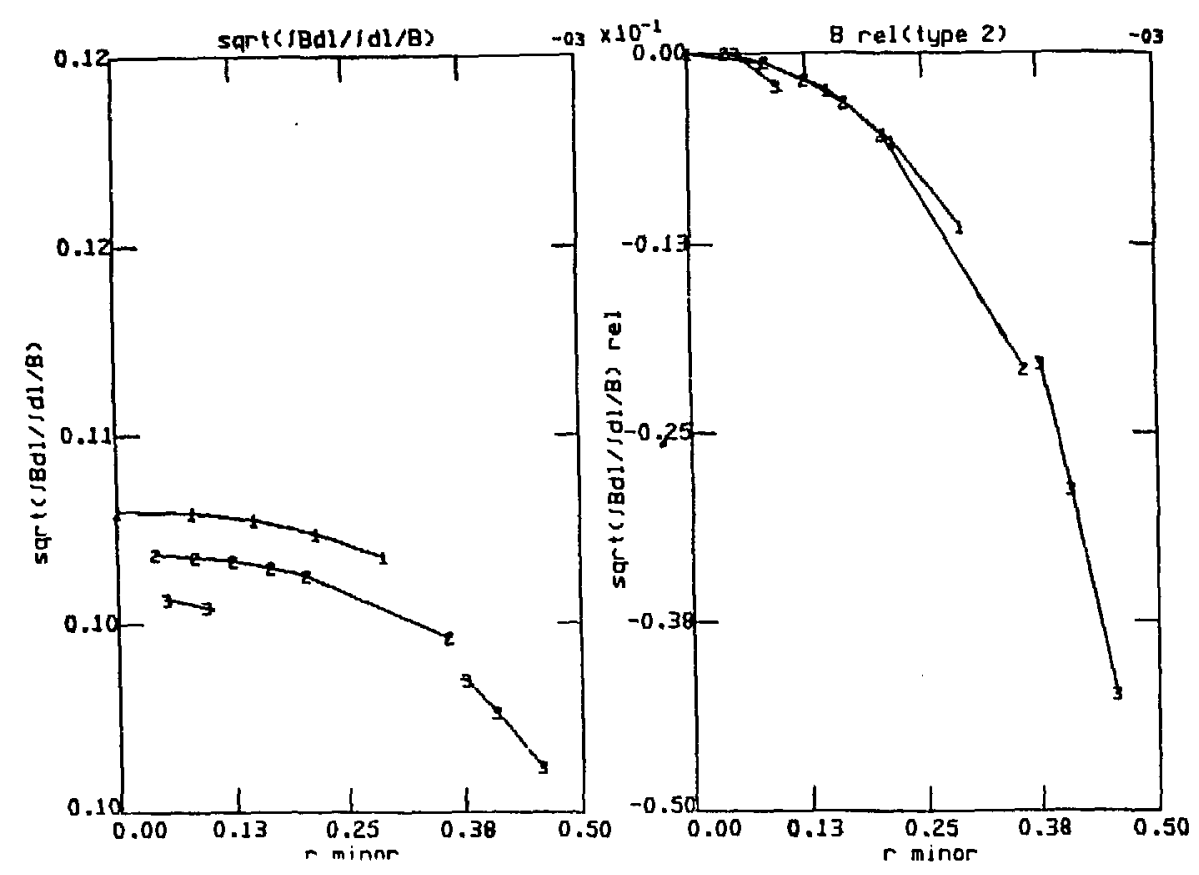

Fig. 6c. $<B>$ and $B_{\text {rel }}$ (type 2) (otherwise, same as Fig. 6a).
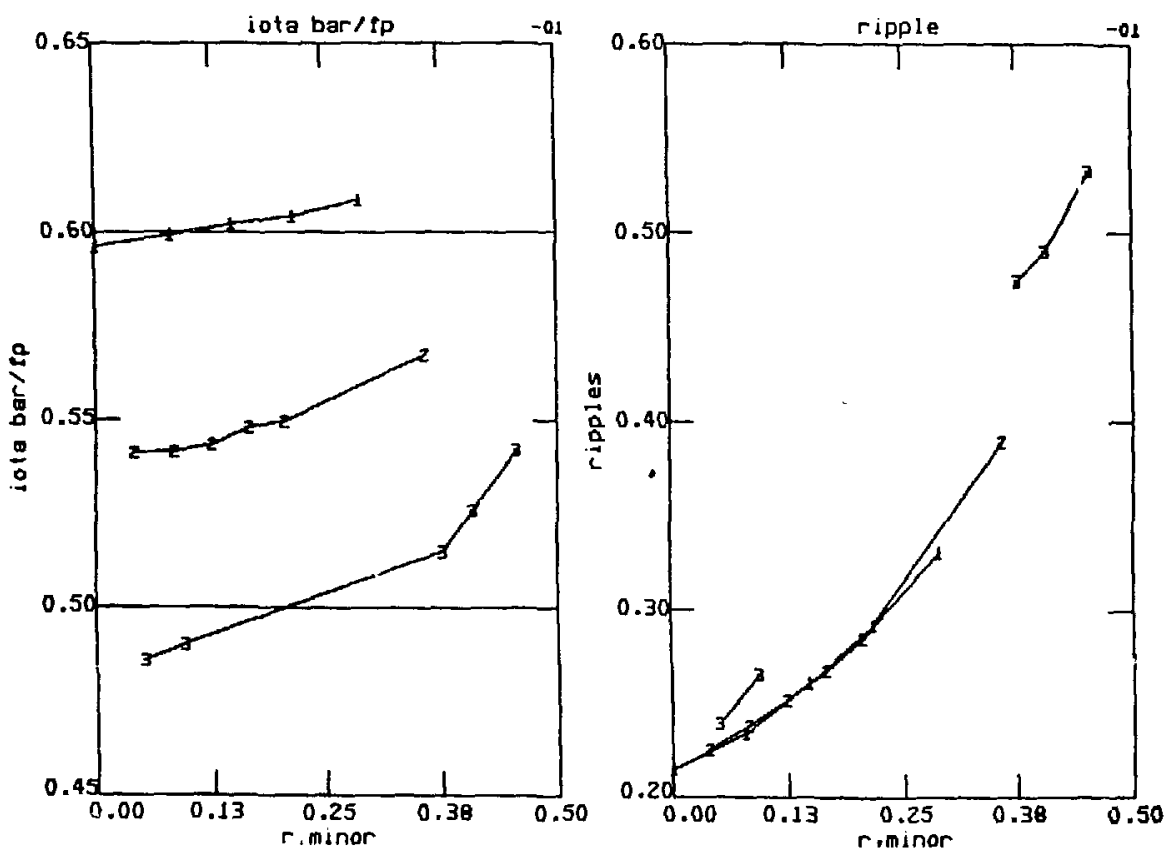

Fig. 6d. Iota Bar per Field Period and Ripple (otherwise, same as Fig. 6a). 


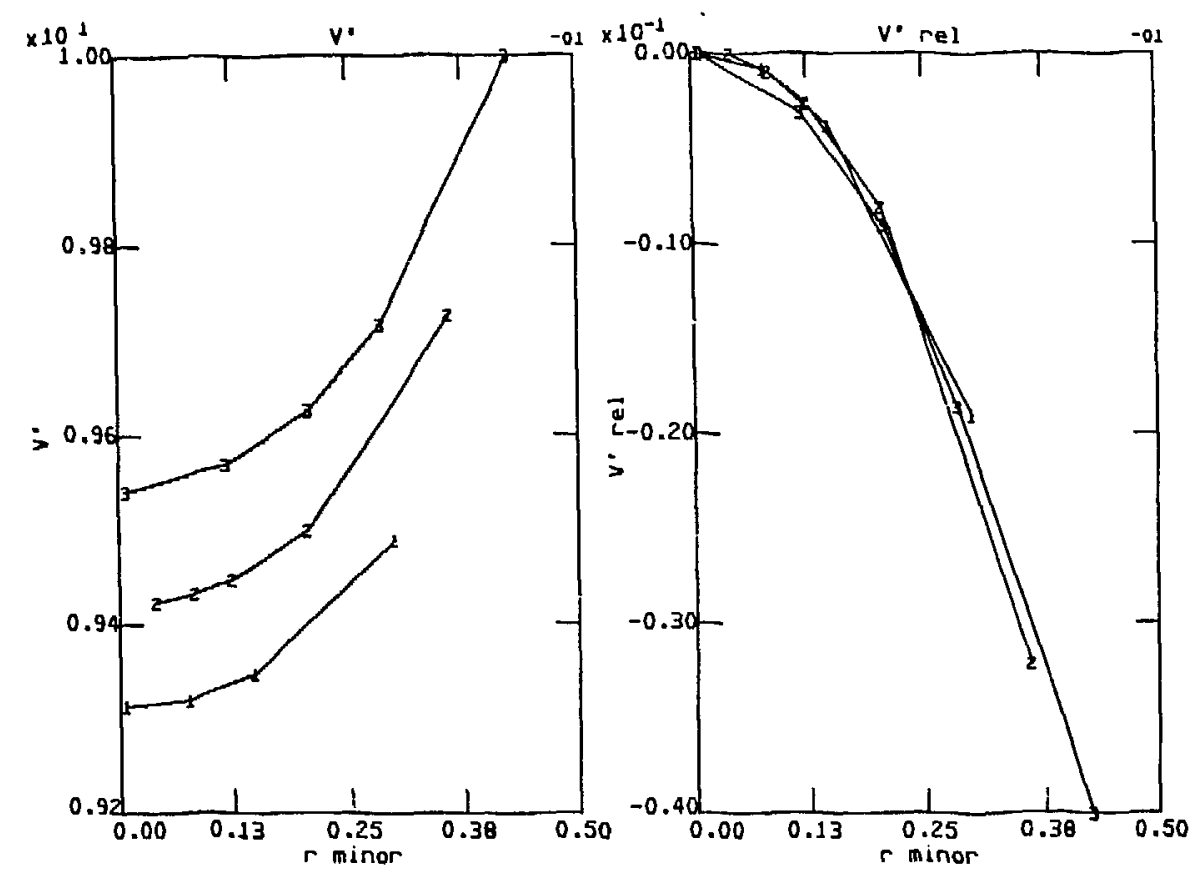

Fig. 7a. $V$ and $V^{\prime}$ rel: The Effects of $c_{3}$ Lateral Deformation on Magnetic Profile, Rotational Transform, and Ripple (2\% B vertical).

Curve $\# 2-c_{3}$ at nominal value of 1.2

Curve \#1 - $0.9 \times$ nominal, and

Curve \#3- 1.1 x nominal.

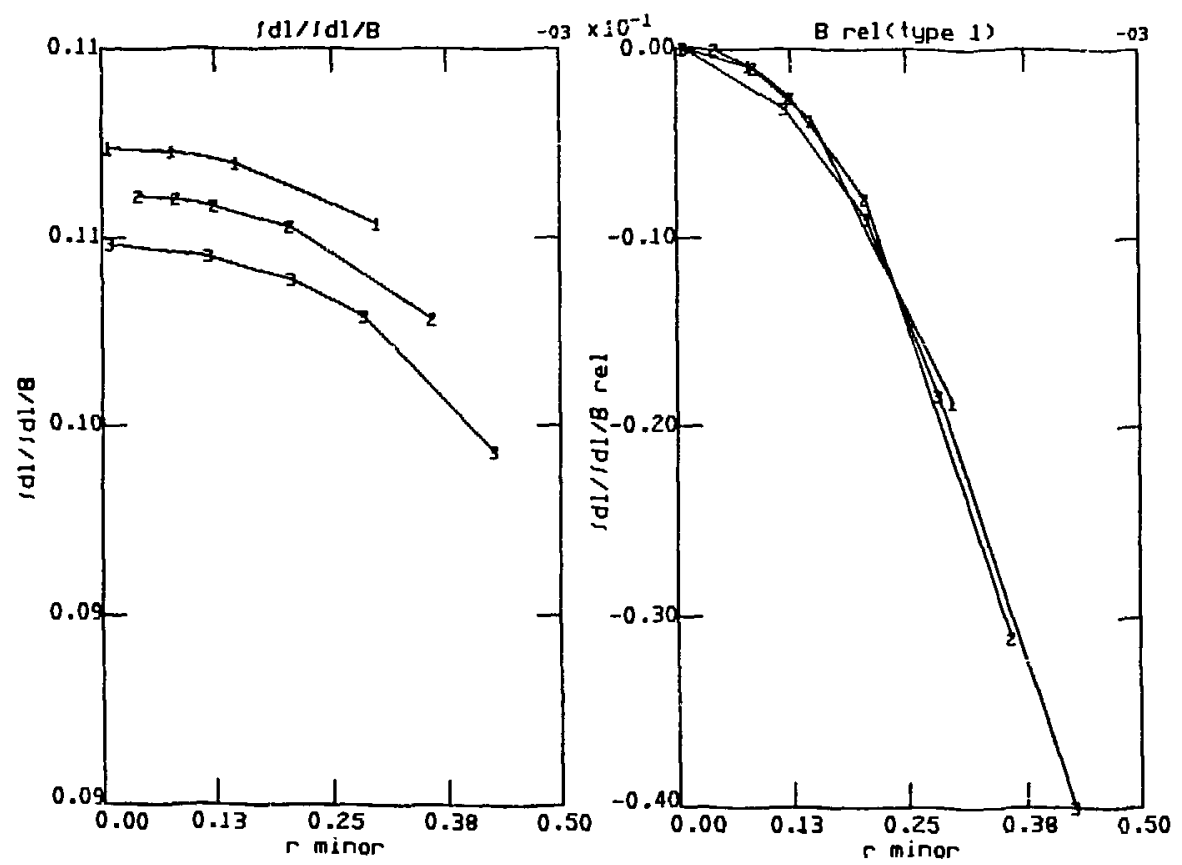

Fig. Fb. <B> and $B_{\text {rel }}($ type. 1) (otherwise, same as Fig. 7a). 

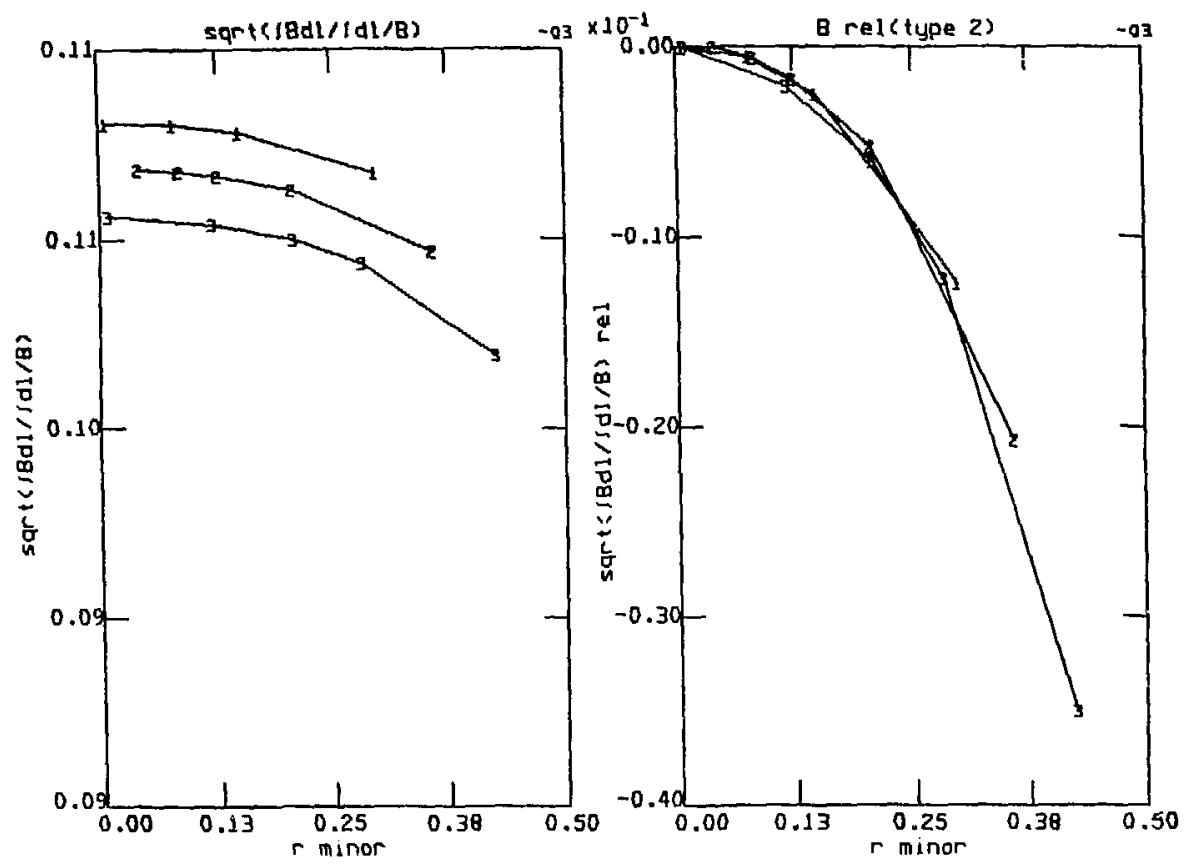

Fig. $7 c .<B>$ and $B_{\text {rel }}$ (type 2) (otherwise, same as Fig. 7a).
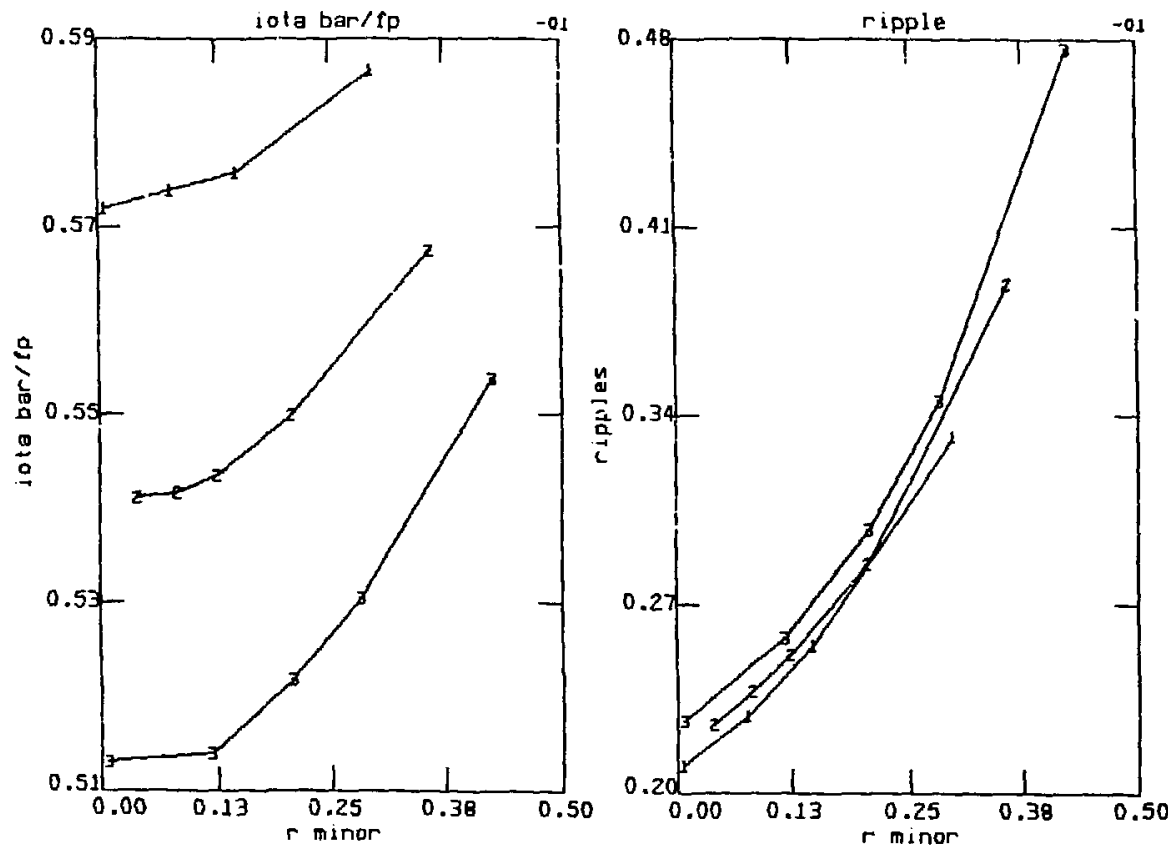

Fig. 7d. lota Bar per Field Period and Ripple (otherwise, same as Fig. 7a). 

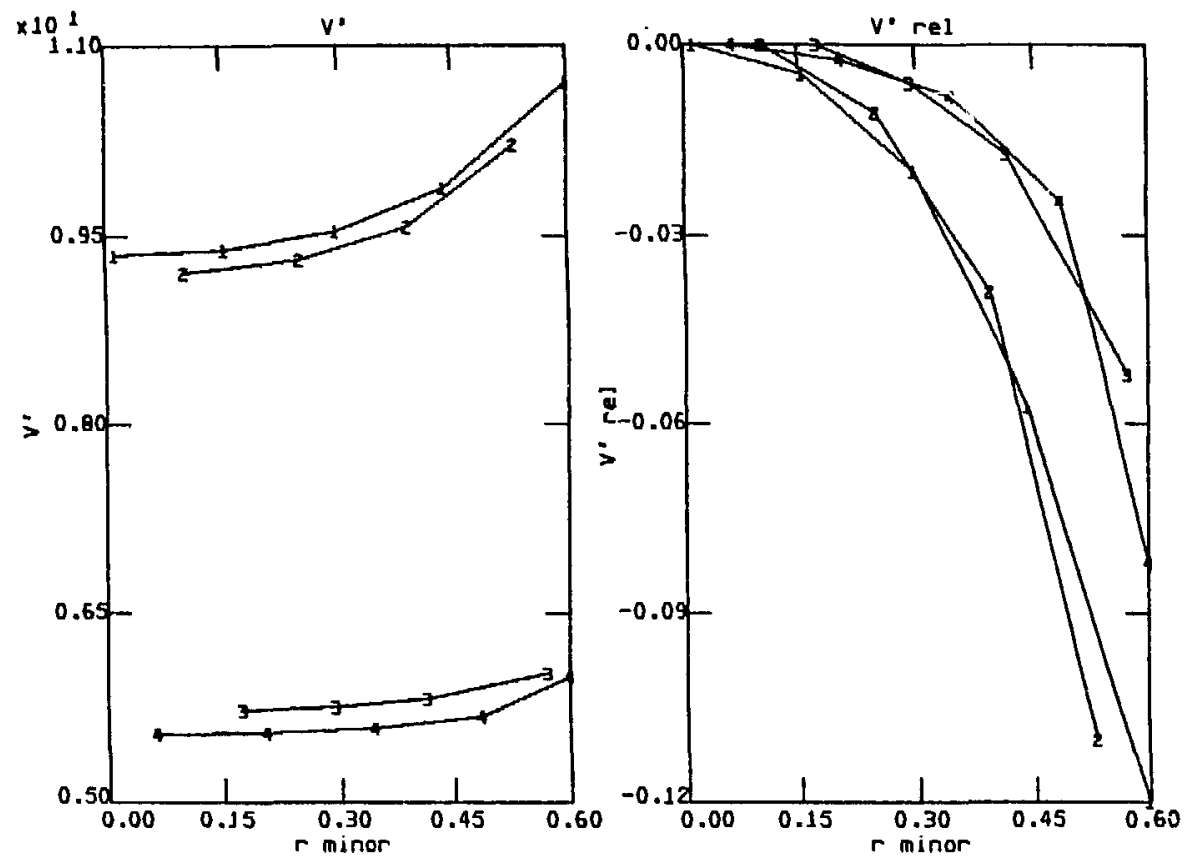

Fig. Ba. V' and $V^{\prime}$ rel: Comparison of Effects of Positive Tilts on 6 and 10 Coils per Field Period ( $B_{\text {vert }}=2 \% B_{\text {tor }}$ ).

6 Coils per Field Period:

Curve \#1 - plus 10 degrees tilt from vertical,

Curve $\# 2$ - plus 5 degrees tilt from vertical,

10 Coils per Field Period:

Curve \#3 - plus 10 degrees tilt from vertical, and

Curve \#4 - plus 5 degrees tilt from vertical.
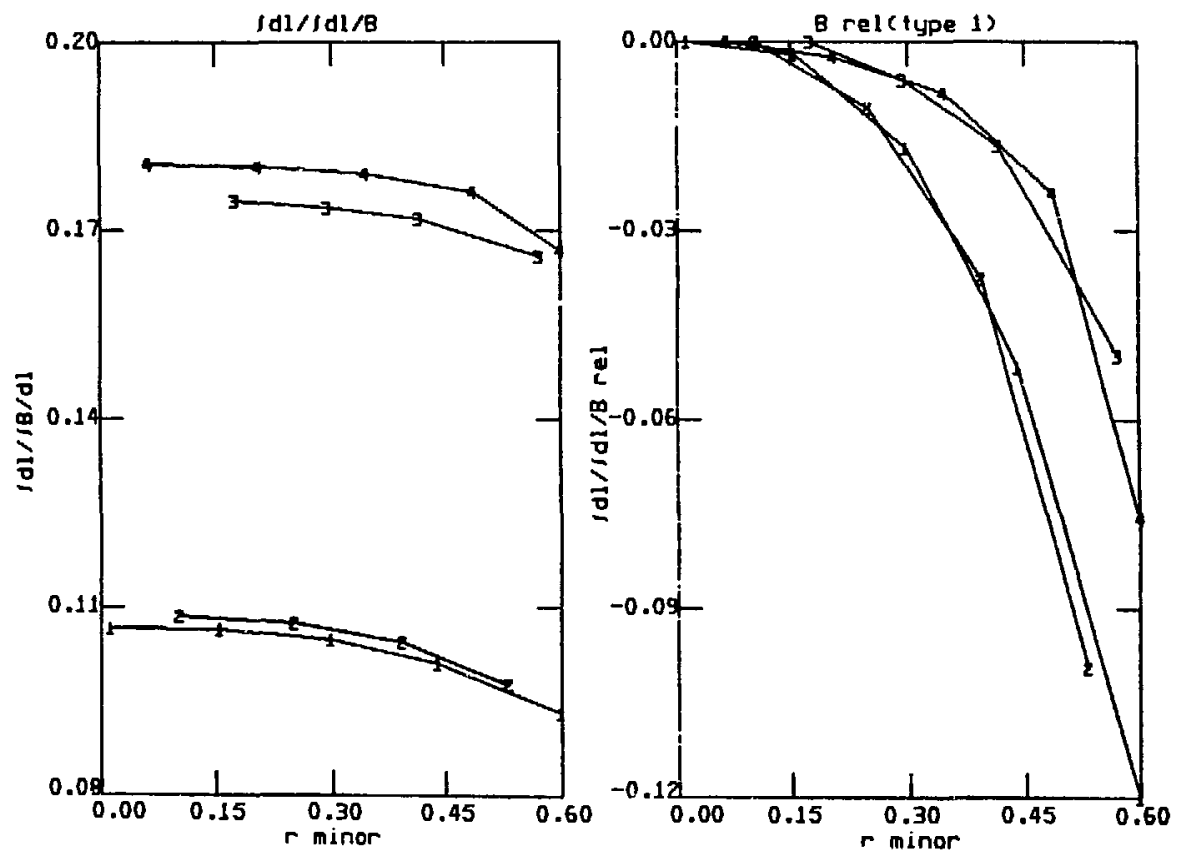

Fig. 8b. <B> and $B_{\text {rel }}$ (type 1) (otherwise, same as Fig. 8a). 

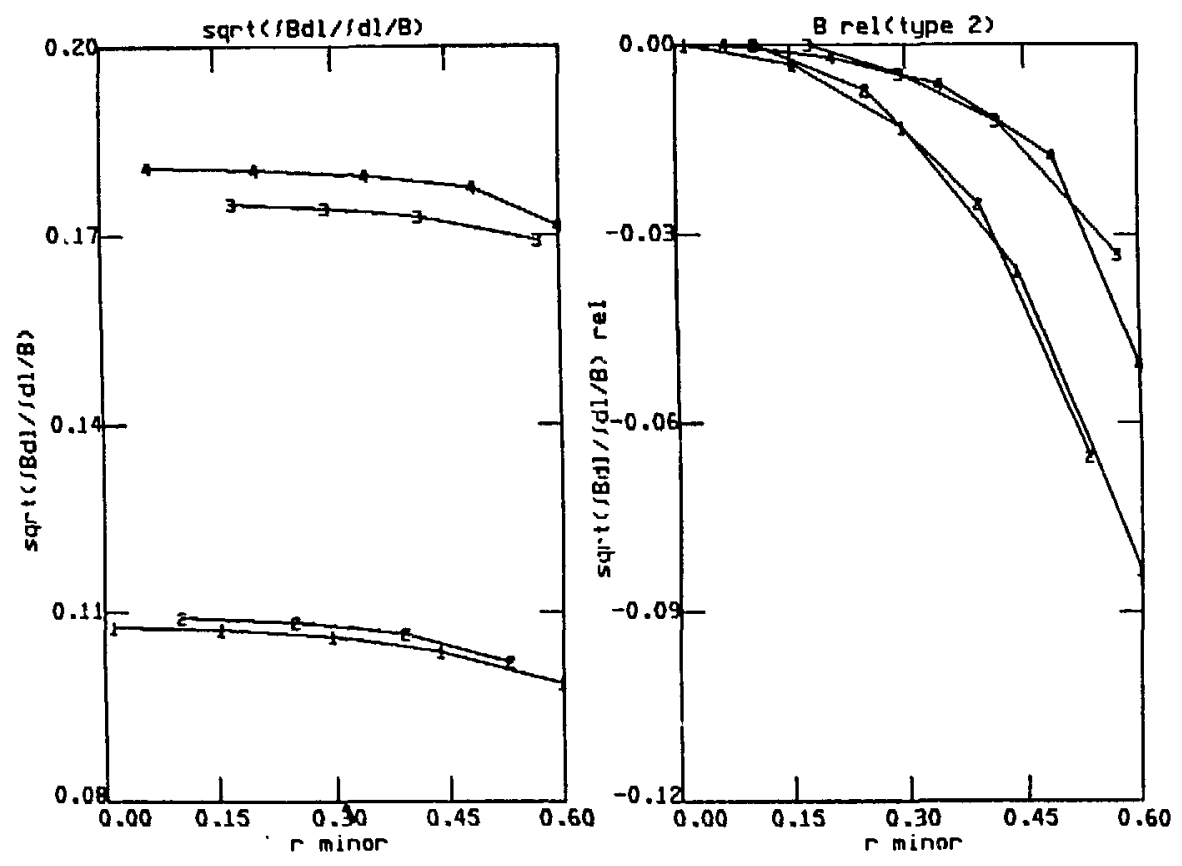

Fig. $8 c . \quad\left\langle B>\right.$ and $B_{\text {rel }}$ (type 2) (otherwise, same as Fig. 8a).
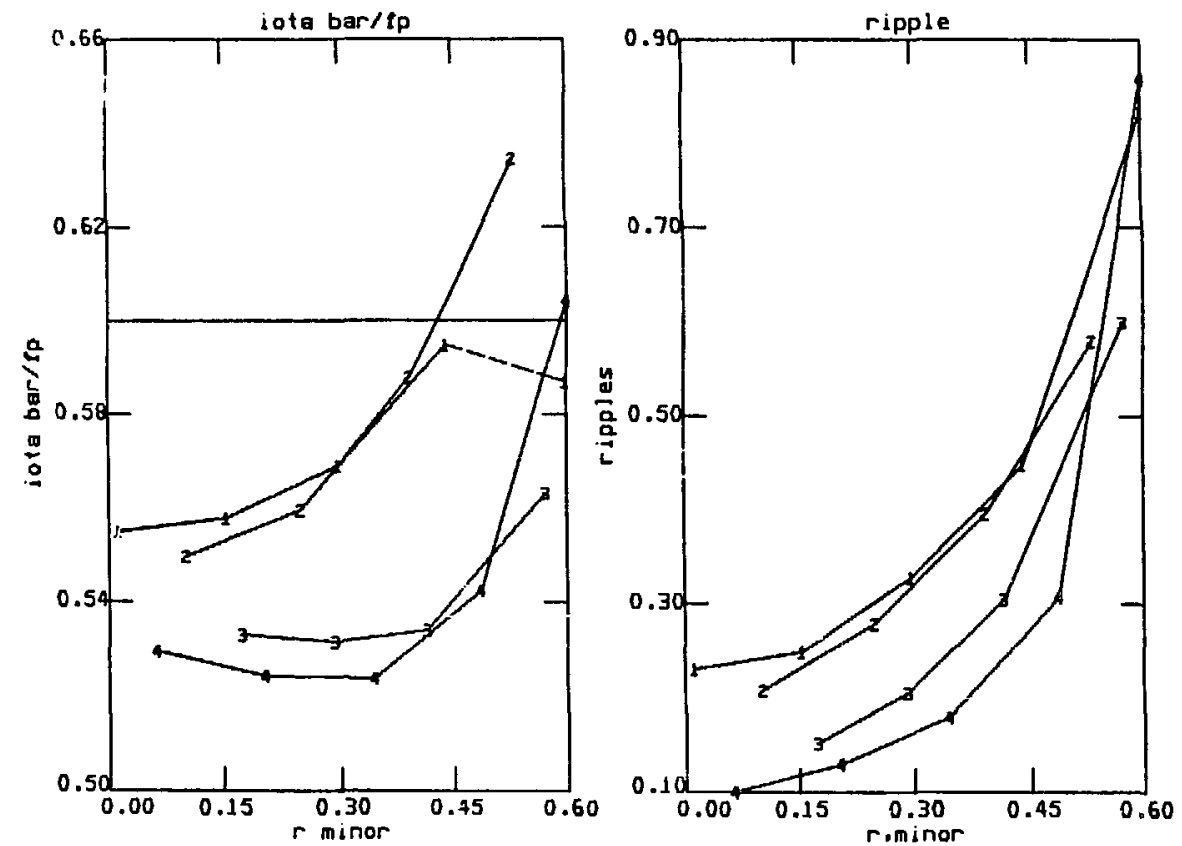

Flg 8d. lota Bar per Field Period and Ripple (otherwise, same as Fig. 8a). 

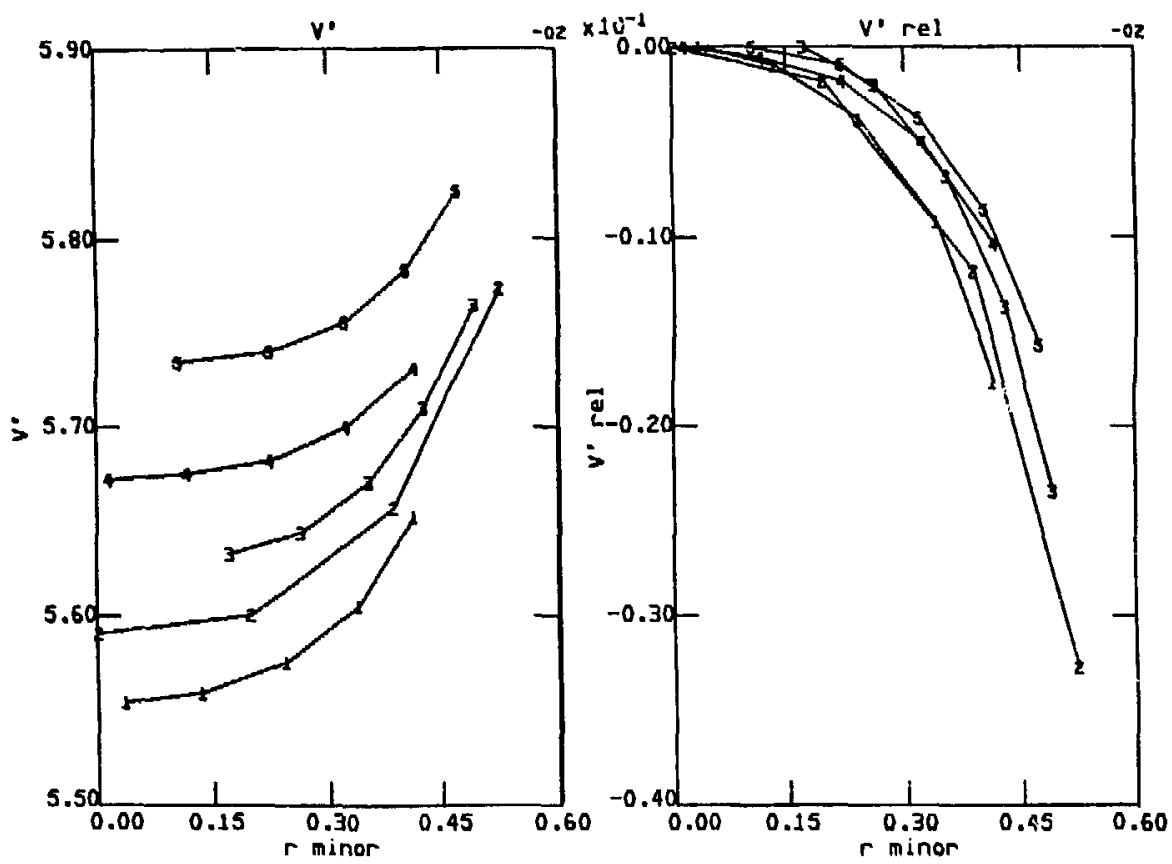

Fig. 9a. $V^{\prime}$ and $V^{\prime} r e l$ : Effects of Aspect Ratio on Magnetic Profiles, Rotational Transform, and Ripple ( $B_{\text {vert }}=2 \% B_{\text {tor }}+5$ degrees tilt, $10 \mathrm{coils} / \mathrm{field}$ period - otherwise, same as before).

$$
\begin{aligned}
& \frac{\text { Curve }}{1} \quad \frac{\mathrm{r}_{\mathrm{b}}{ }^{*}}{1.04} \quad \frac{\mathrm{A}_{\text {coil }}}{9.53} \\
& 2 \quad 1.09 \quad 9.09 \text { (h.B.v.) } \\
& 3 \quad 1.14 \quad 8.70 \\
& 4 \quad 1.21 \quad 8.16 \\
& \begin{array}{lll}
5 & 1.28 & 7.77
\end{array}
\end{aligned}
$$

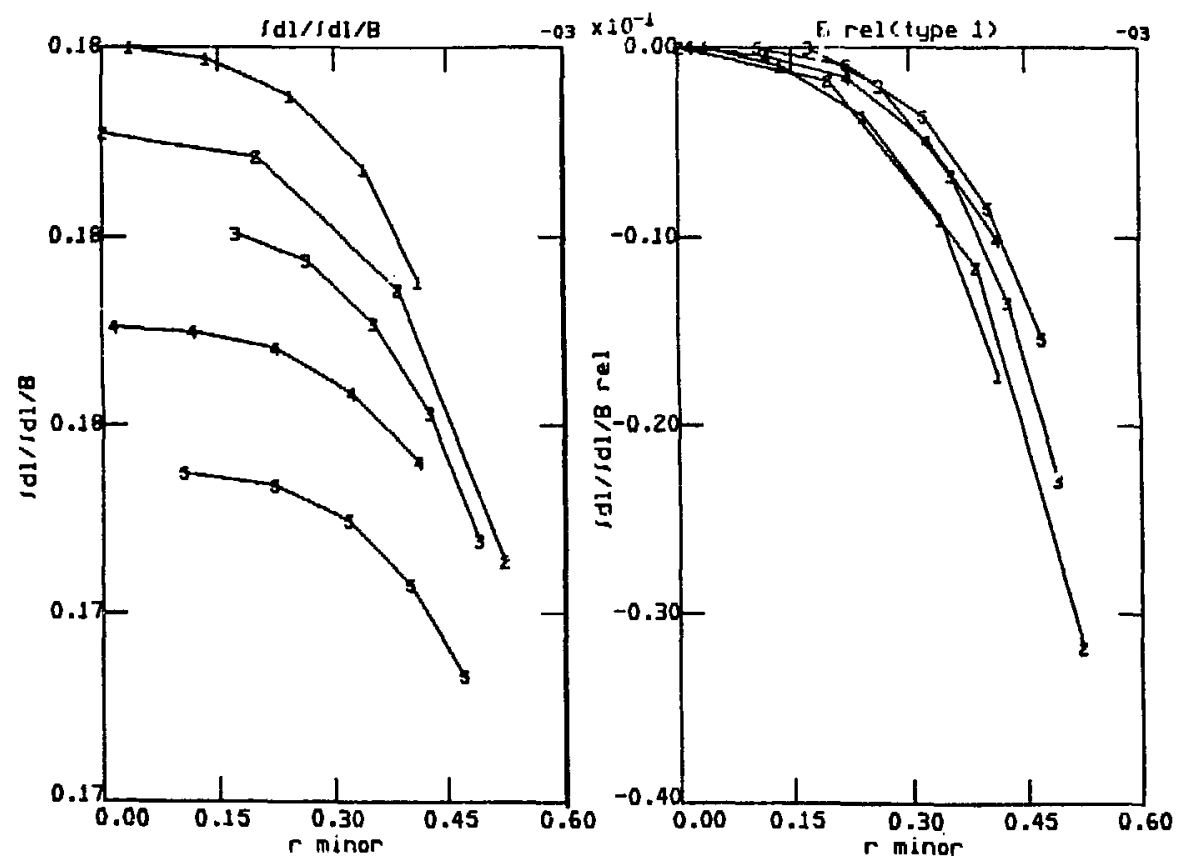

Fig. 9b. $<B>$ and $B_{\text {rel }}$ (type 1) (otherwise, same as Fig. 9a). 


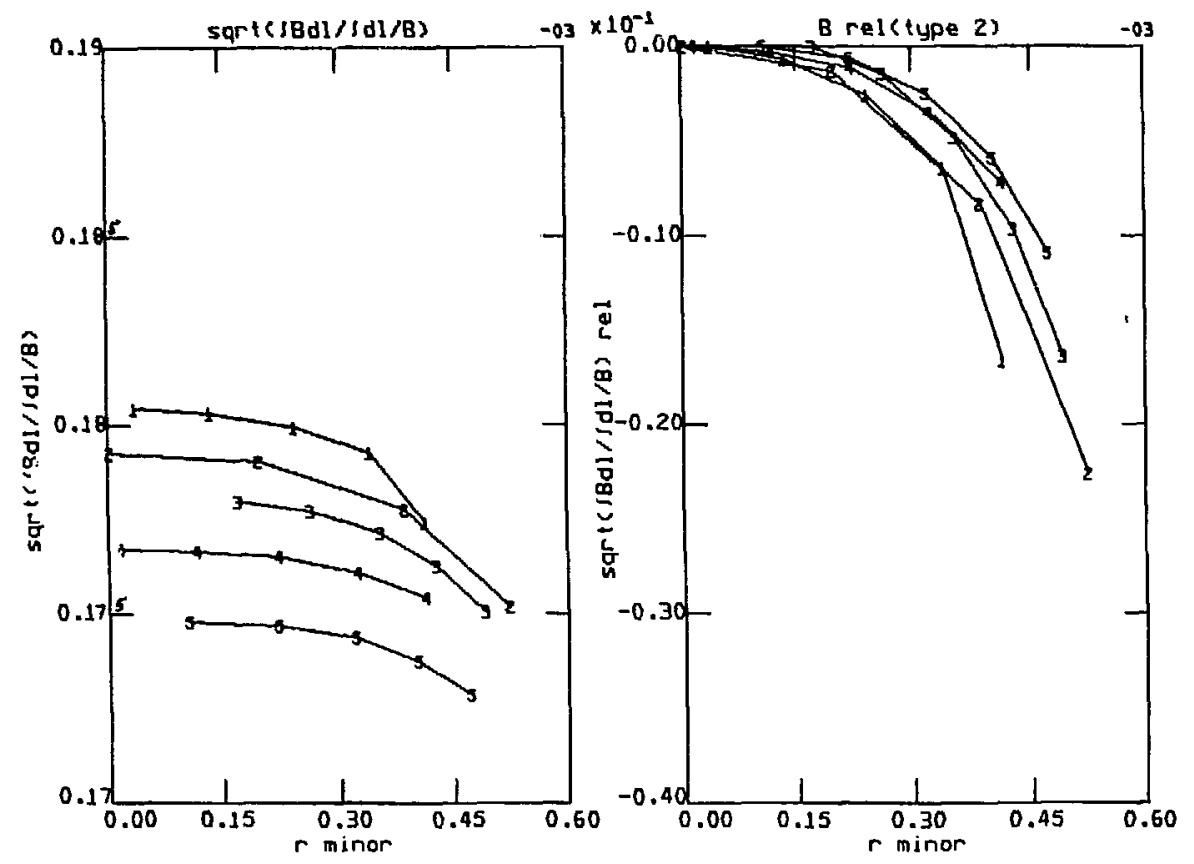

Fig. 9c. $<B>$ and $B_{r e l}$ (type 2) (otherwise, same as Fig. 9a).
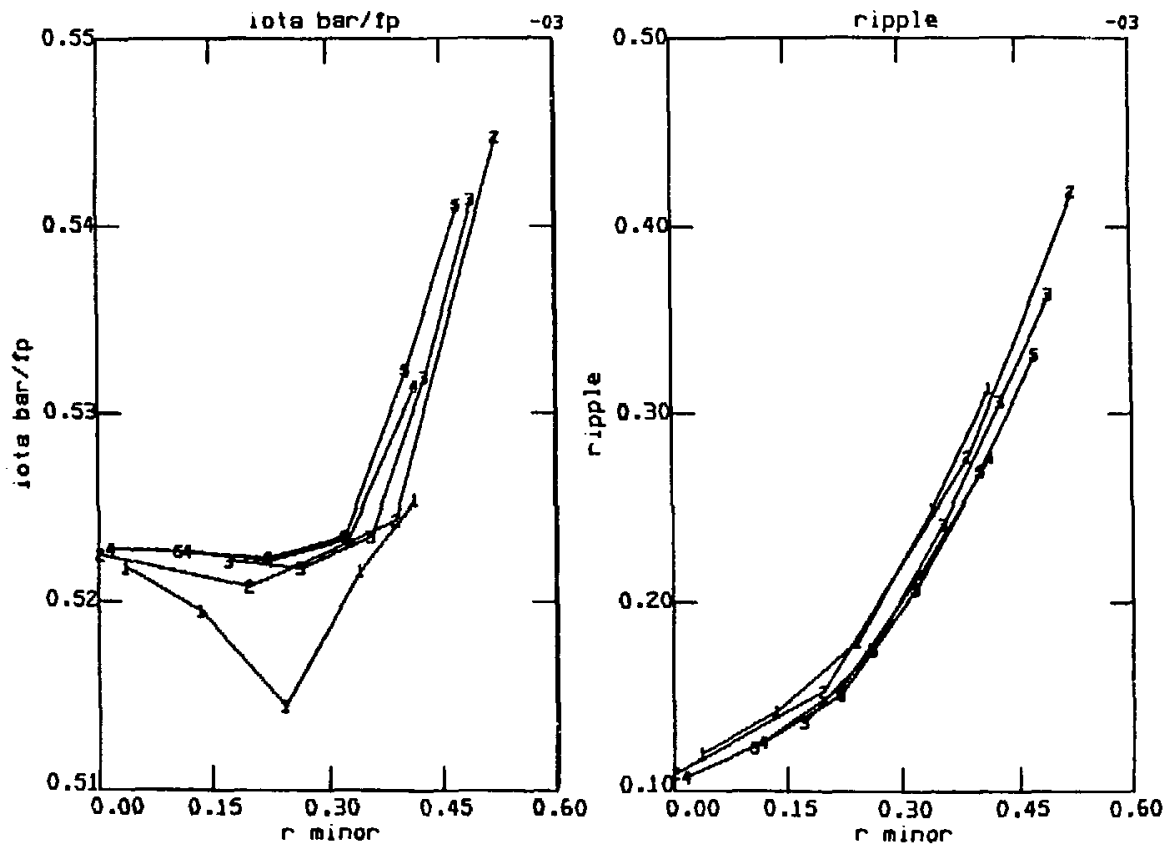

Fig. 9d. Iota Bar per Field Period and Ripple (otherwise, same as Fig. 9a). 

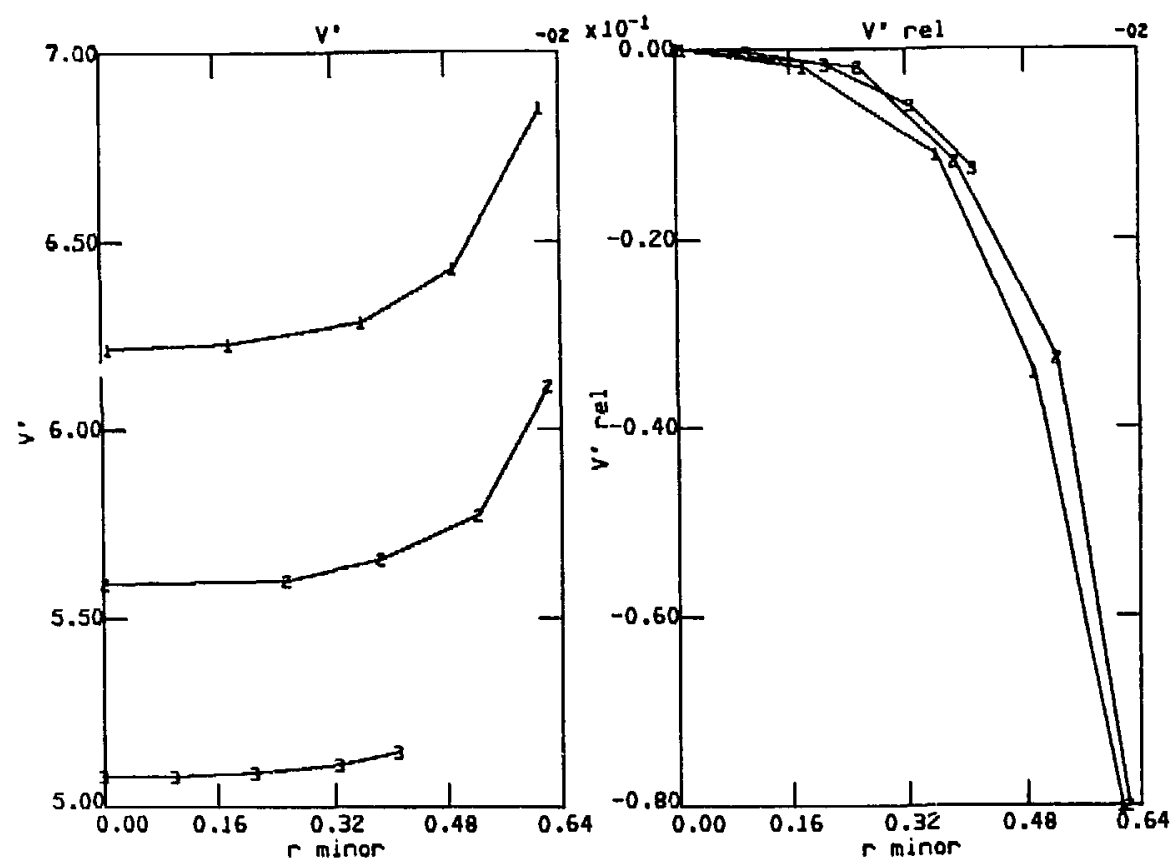

Fig. 10a. $V$, and $V^{\prime}$ rel: Effects of 9,10 and 11 Coils per Field Period on Magnetic Profiles, Rotational Transform and Ripple ( $2 \%$ B vertical, +5 degrees tilt - otherwise, same as before).

Curve 1: 9 Coils/Field Period

Curve 2: 10 Coils/Field Period

Curve 3: 11 Coils/Field Period
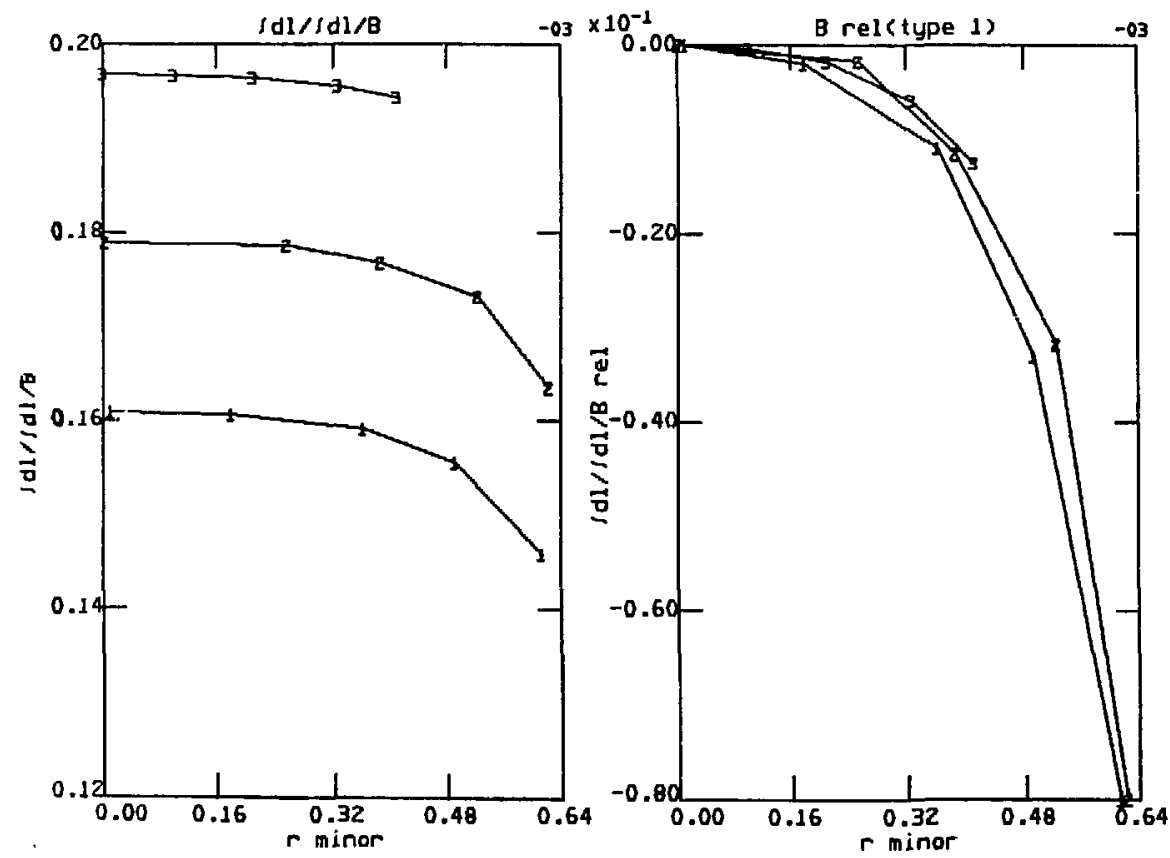

Fig. 10b. $<\mathrm{B}>$ and $\mathrm{B}_{\text {rel }}$ (type 1) (otherwise, same as Fig. 10a). 


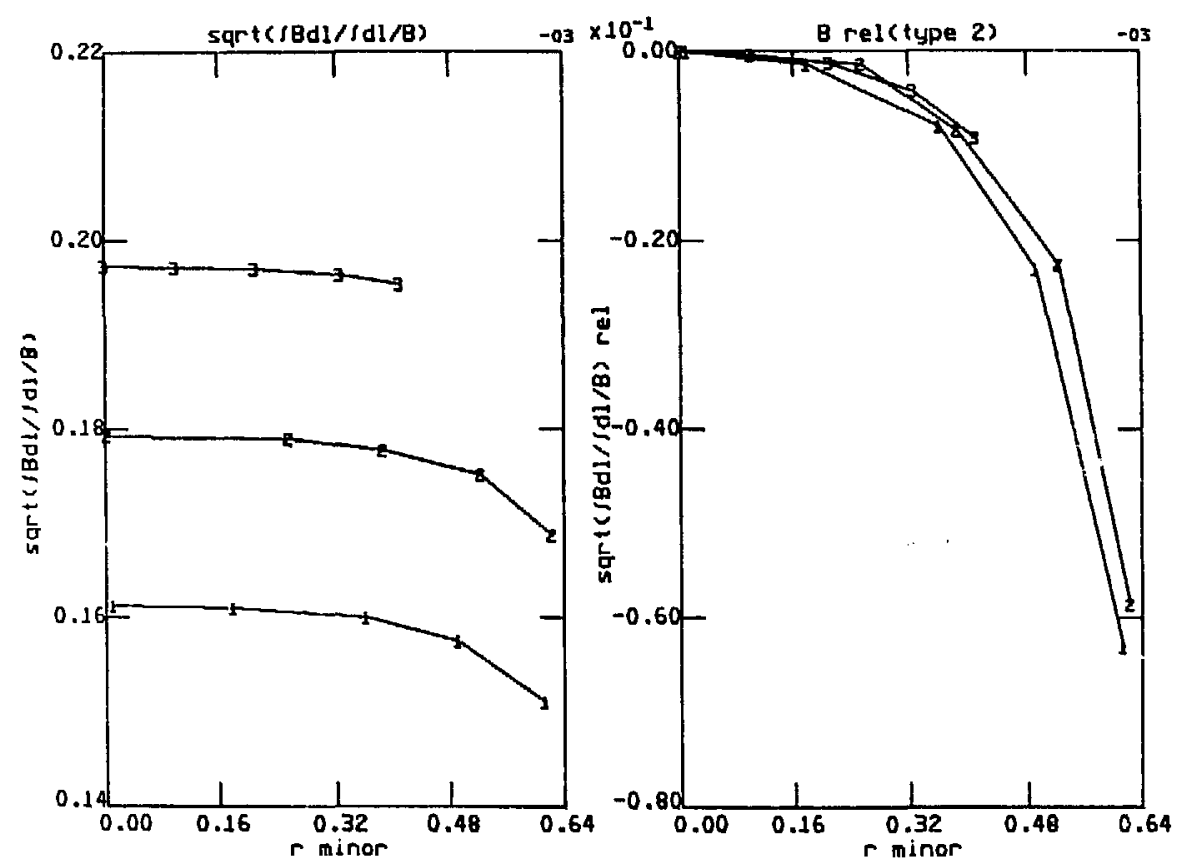

Fig. 10c. <B> and $B_{\text {rel }}$ (type 2) (otherwise, same as Fig. 10a).
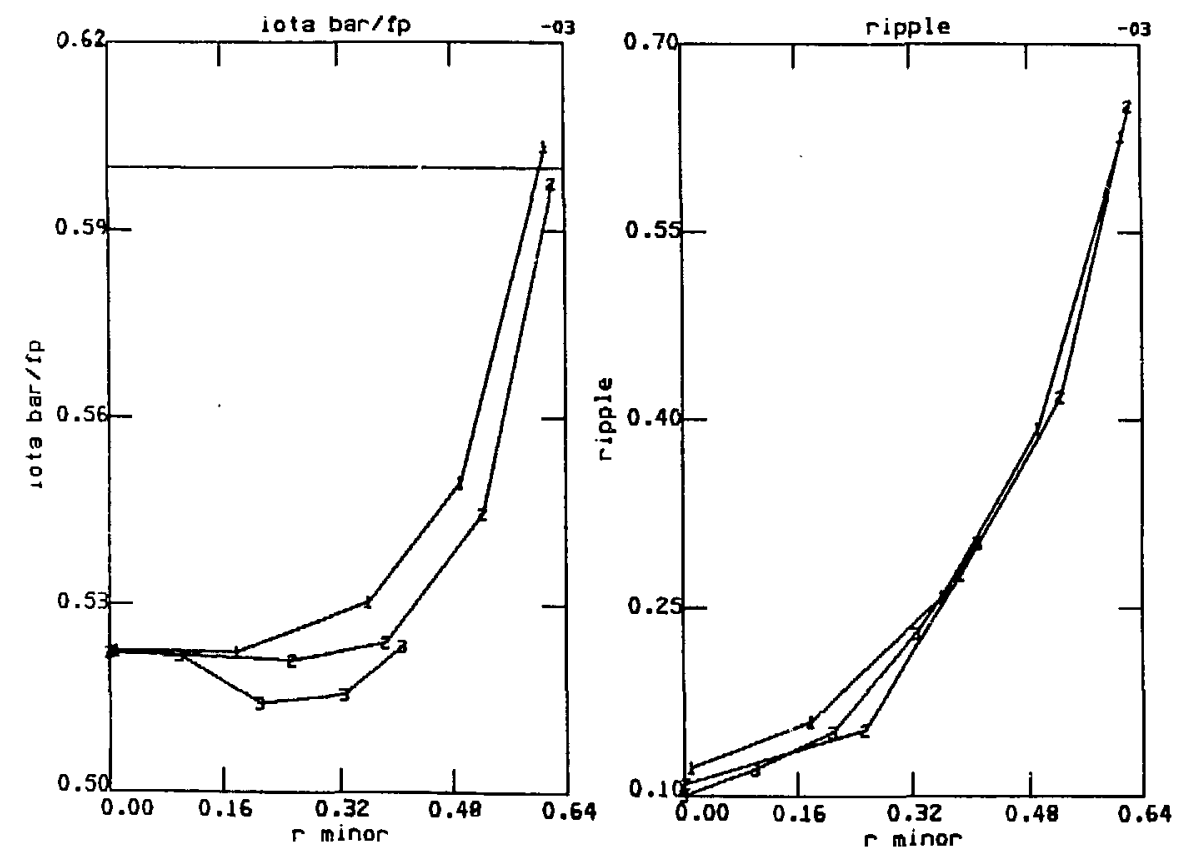

Fig. 10d. Iota Bar per Field Period and Ripple (otherwise, same as Fig. 10a). 


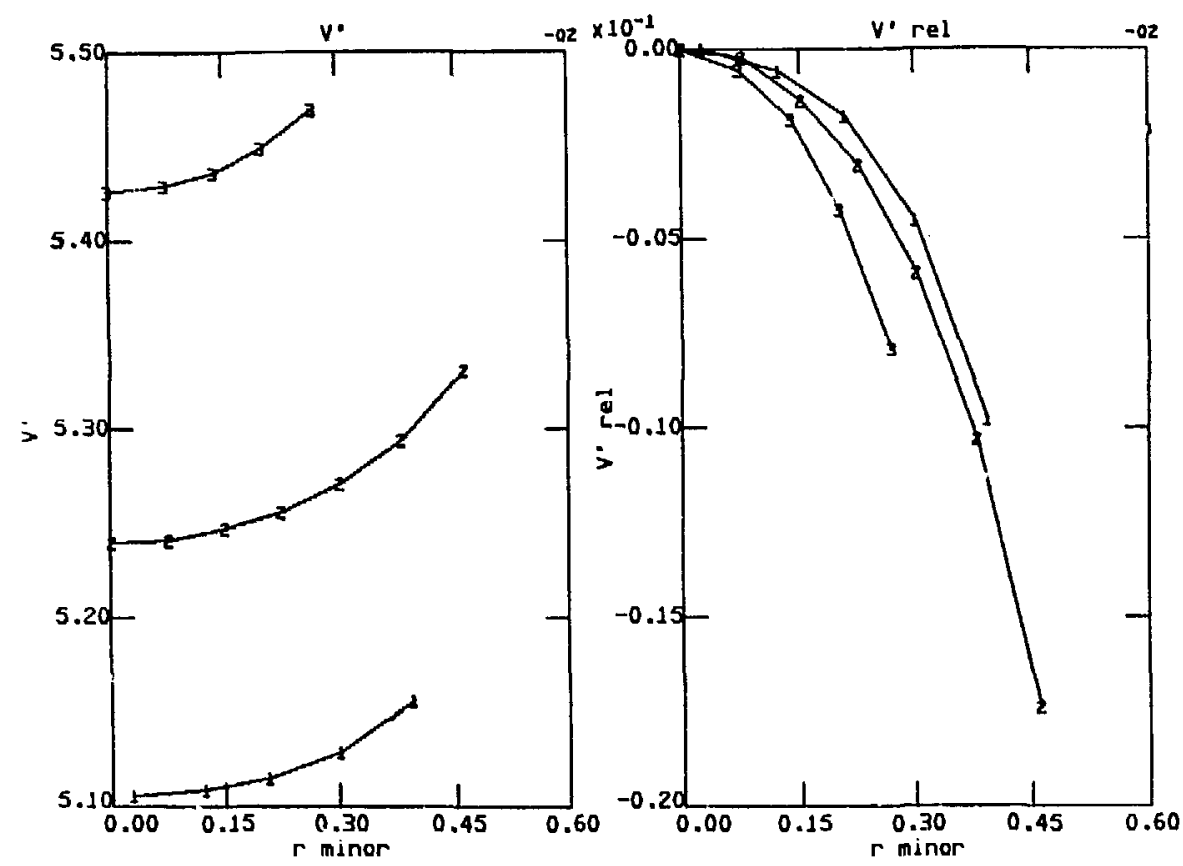

Fig. 11a. $V^{\prime}$ and $V^{\prime}$ rel: Effects of Tilt on Magnetic Profiles, Rotational

Transform, and Ripple (2\% B vertical, 11 coils/field period - otherwise, same as before).
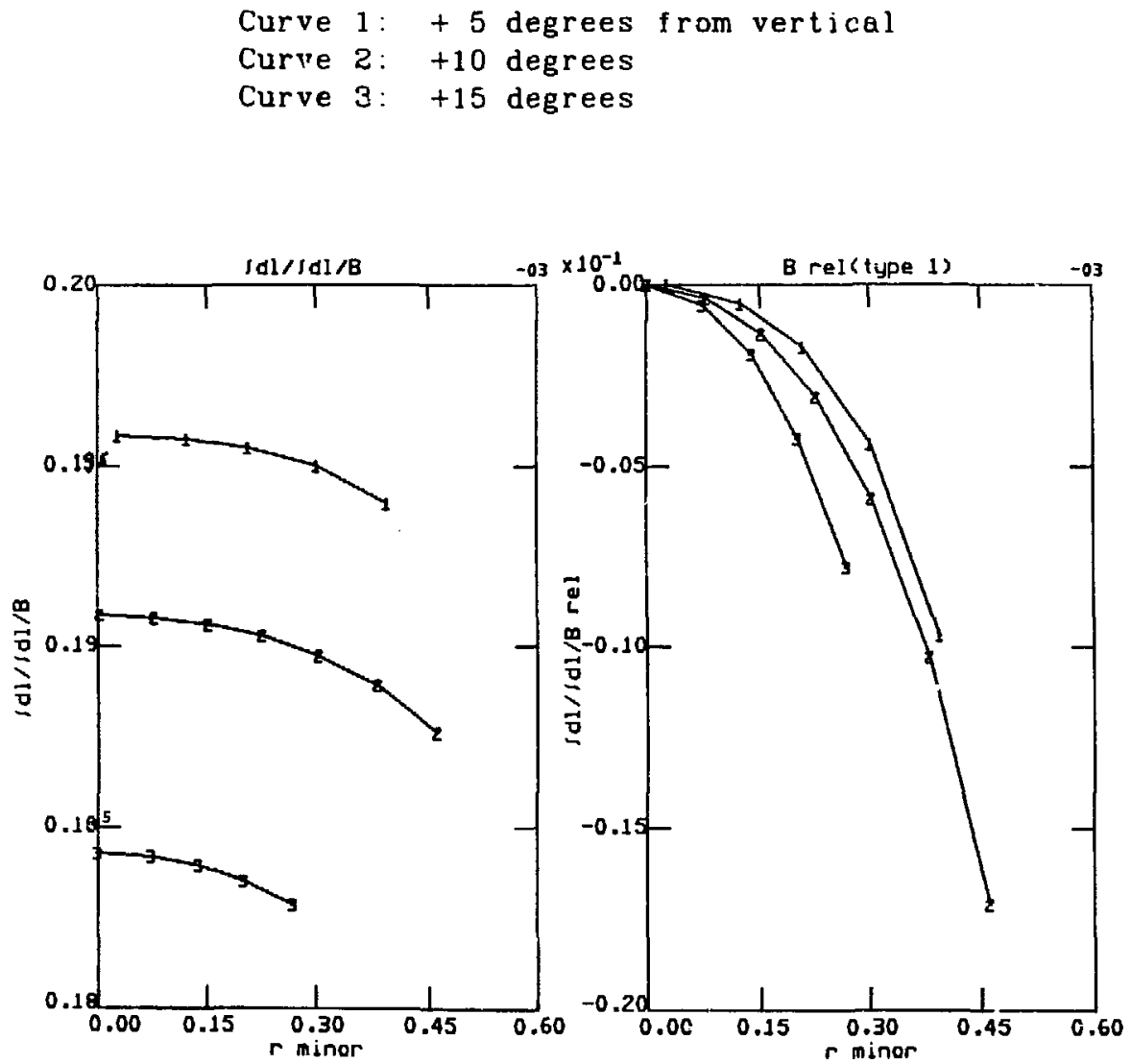

Fig, 11b. $\left\langle B>\right.$ and $B_{\text {rel }}$ (type 1) (otherwise, same as Fig. 11a). 


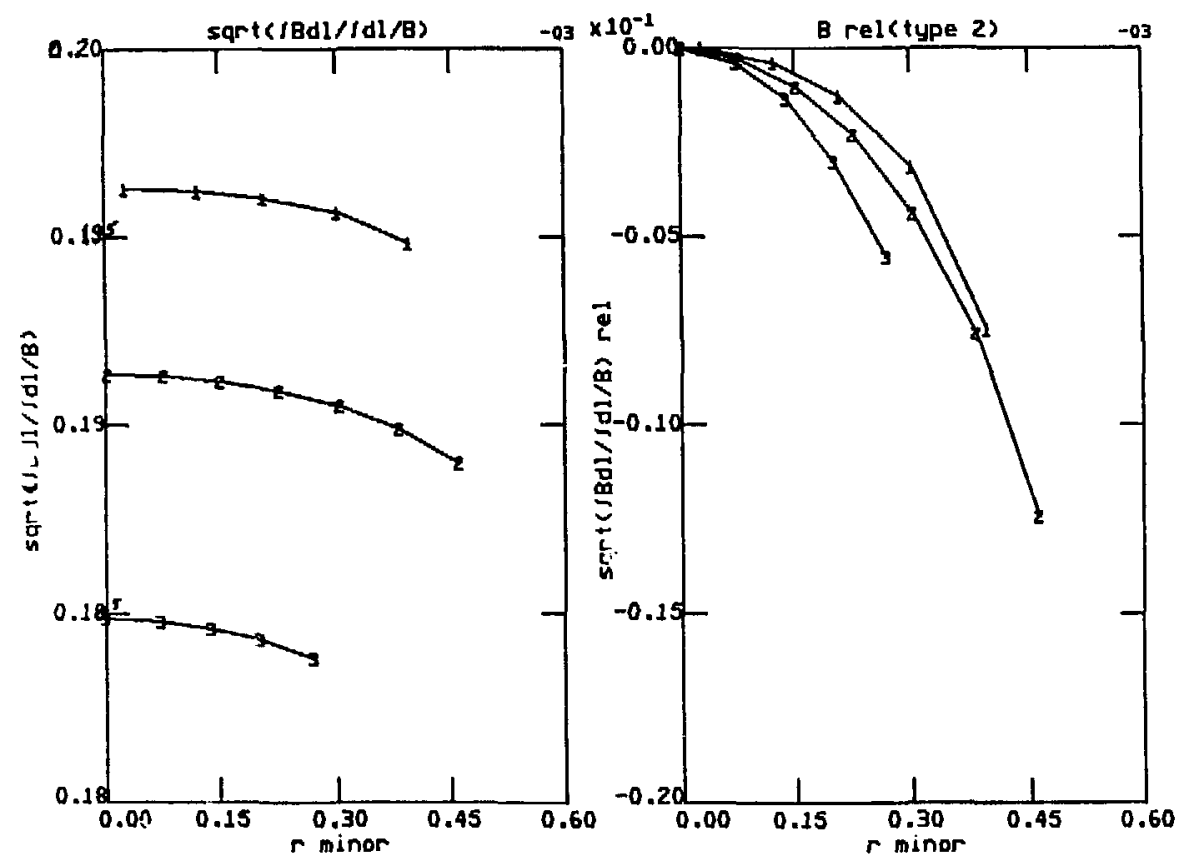

Fig. 11c. $\left\langle B>\right.$ and $B_{\text {rel }}$ (type 2) (otherwise, same as Fig. 11a).
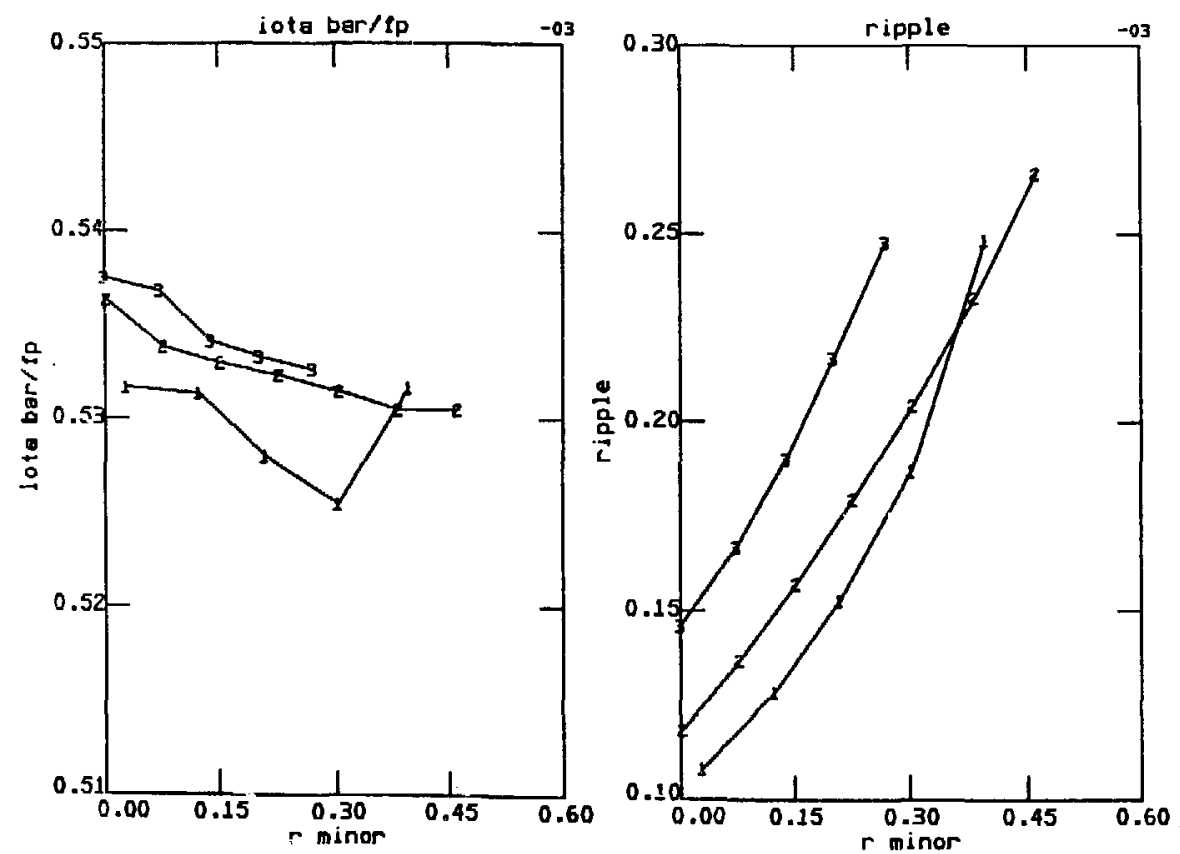

Fig. 11d. Iota Bar per Field Period and Ripple (otherwise, same as Fig. 11a). 

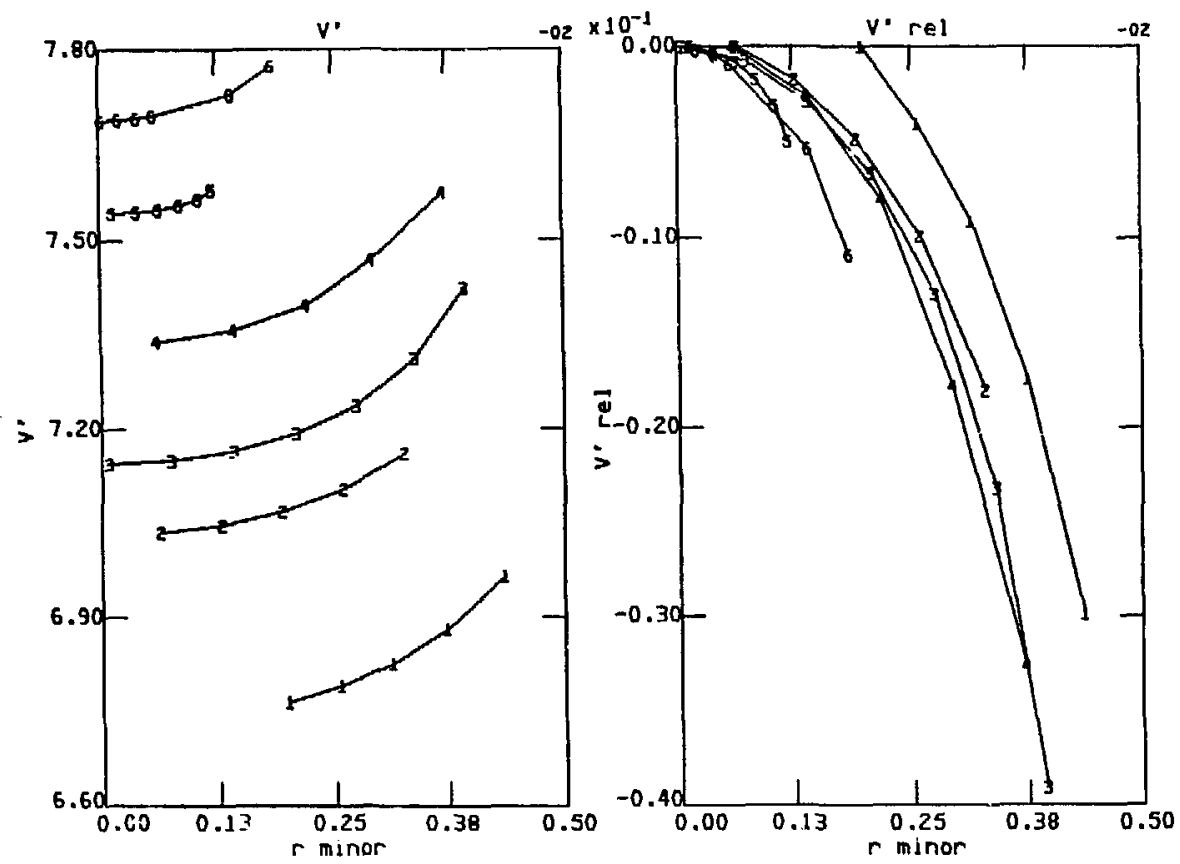

Fig. 12a. $V^{\prime}$ and $V^{\prime}$ rel: Effects of Helical Amplitude on Magnetic Profiles, Rotational Transiorm, and Ripple (Bvert $=2 \% B_{\text {tor }}$. 6 coils/field period, 6 field periods, major radius 6).

\begin{tabular}{|c|c|c|c|c|c|}
\hline Curve & $\Delta_{1}^{*}$ & radius & Curve & $\Delta_{1}^{*}$ & radius $_{\text {gy }}$ \\
\hline 1 & -0.50 & 0.52 & 4 & -0.225 & 0.70 \\
\hline 2 & -0.35 & 0.62 & 5 & -0.15 & 0.76 \\
\hline 3 & -0.30 & 0.66 (h.B.v.) & 6 & -0.10 & 0.80 \\
\hline
\end{tabular}

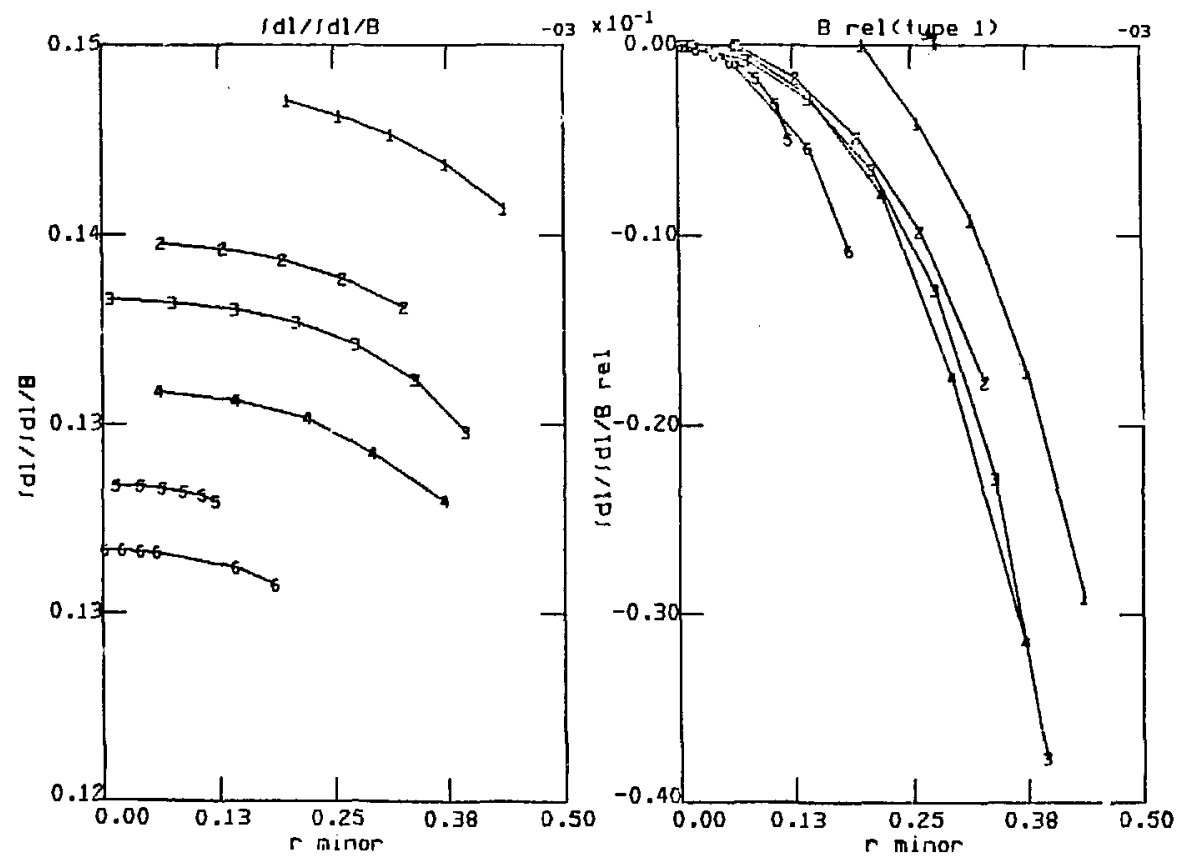

Fig. 12b. $<B>$ and $B_{\text {rel }}$ (type 1) (otherwise, same as Fig. 12a). 


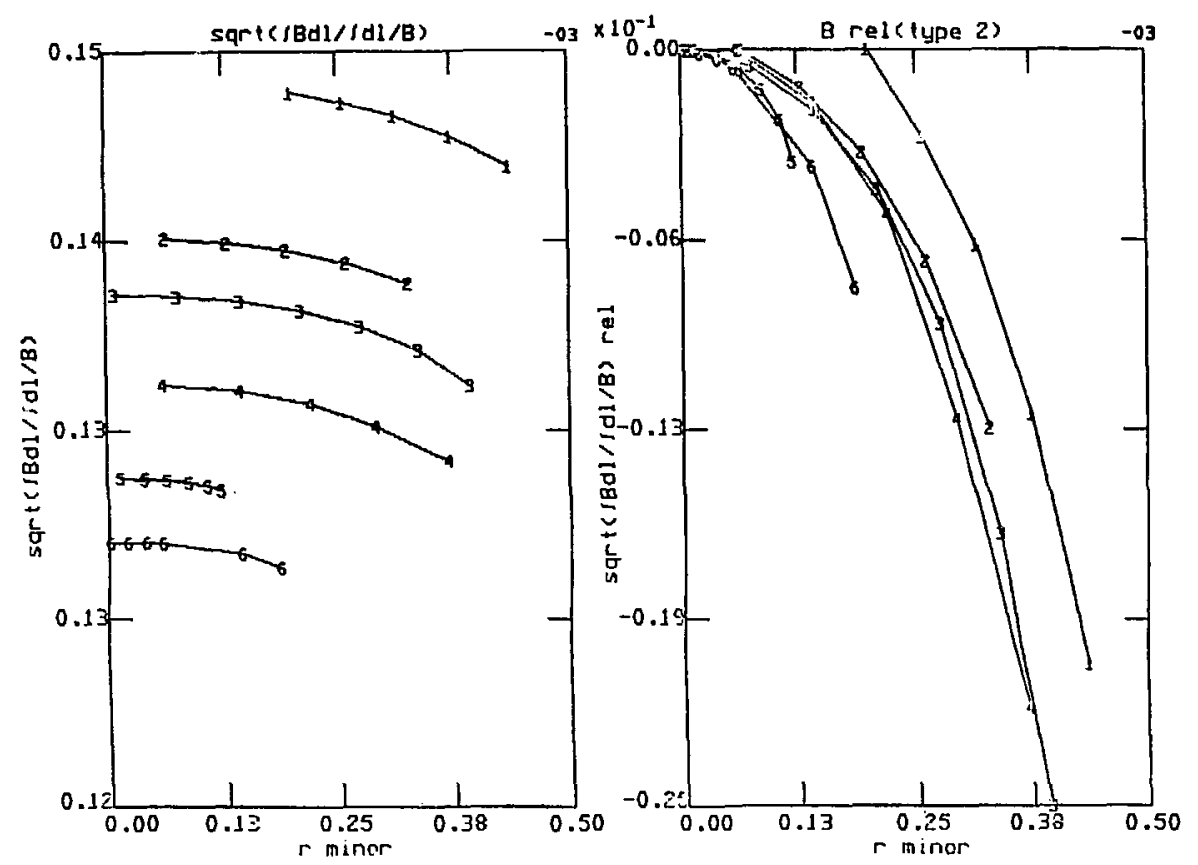

Fig. 12c. <B> and $B_{r e l}$ (type 2) (ctherwise, same as Fig. 12a).
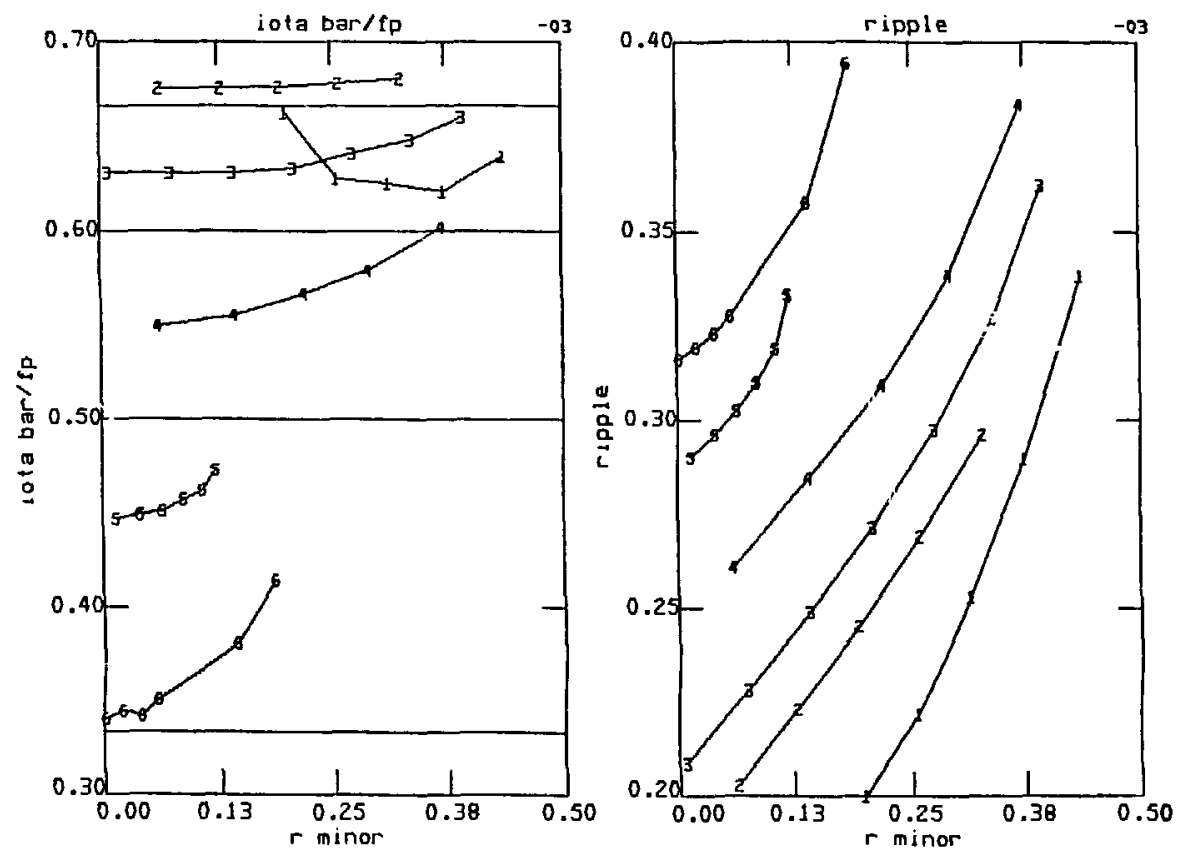

Fig. 12d. lota Bar per Field Period and Ripple (otherwise, same as Fig. 12a). 


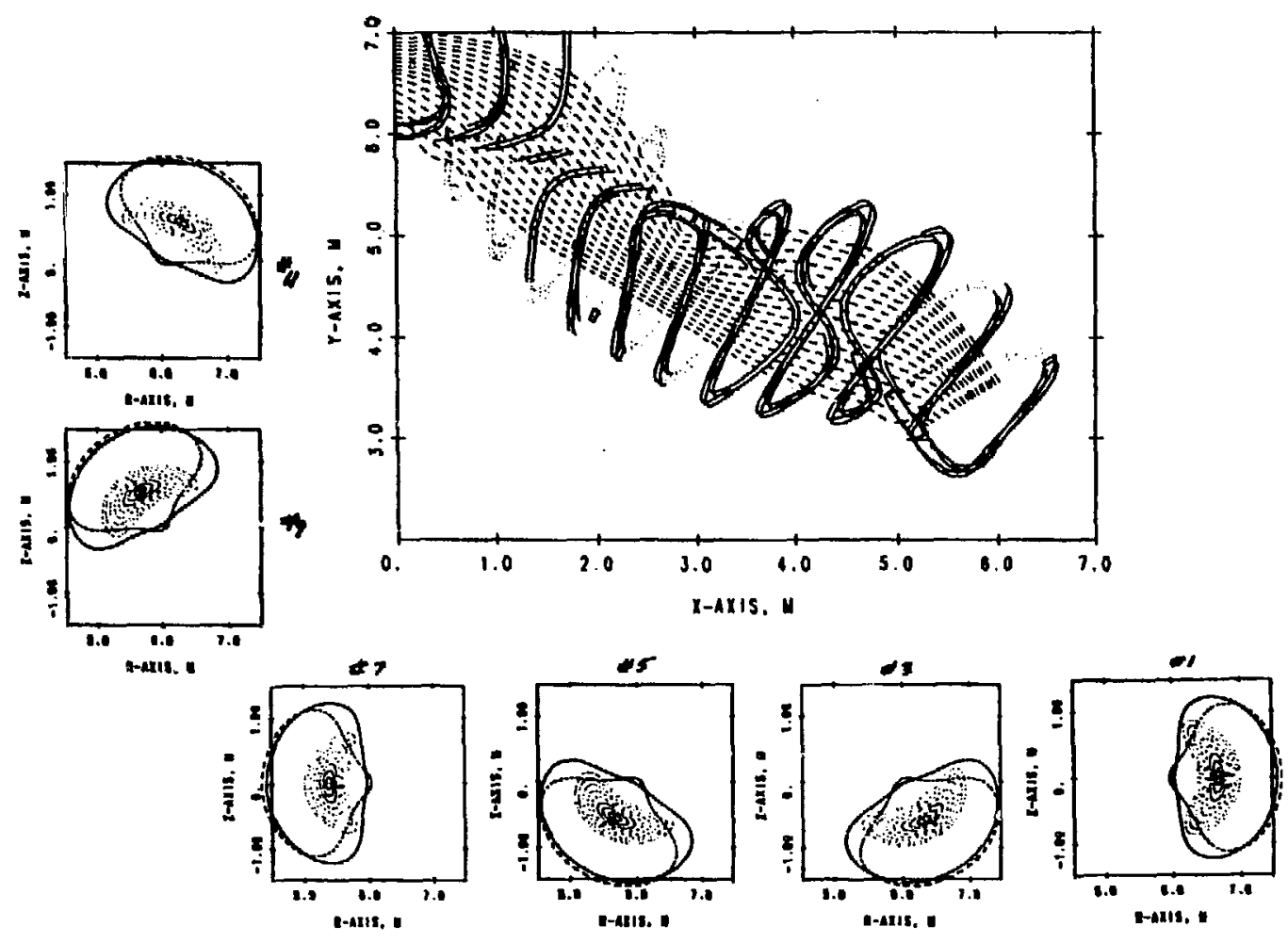

Fig. 13a. Puncture Plots and Top-down View of Coils and Flux Surfaces.

Major radius (a): 6

Coils per field period : 12

B Vertical: 2\% of B toroidal
Field periods (Q): 6

Coil espect ratio (A) : 6 

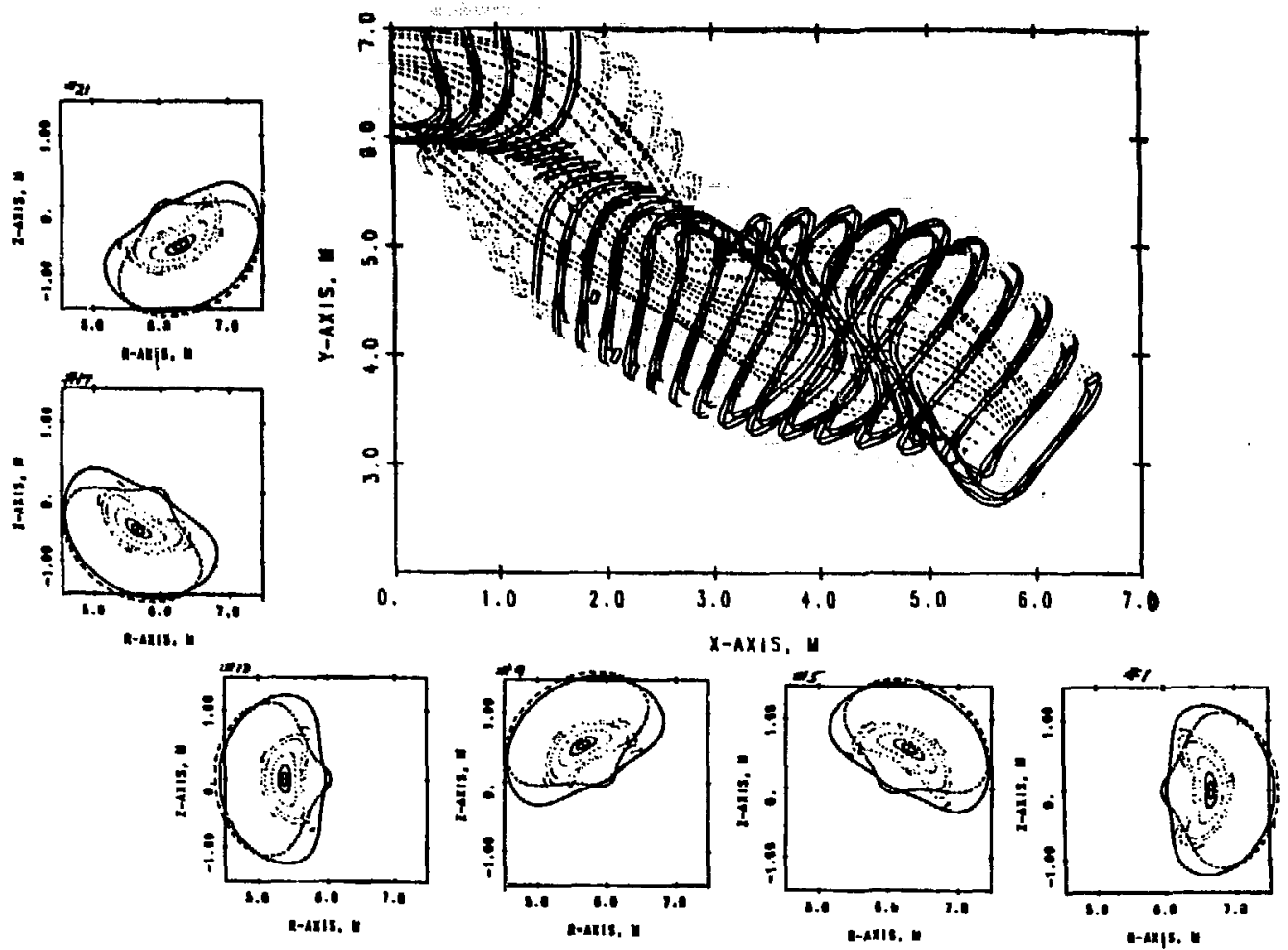

Fig. 13b. Puncture Plots and Top-down View of Coils and Flux Surfaces. Coils per field period : 24 (otherwise, same as Fig. 13a). 

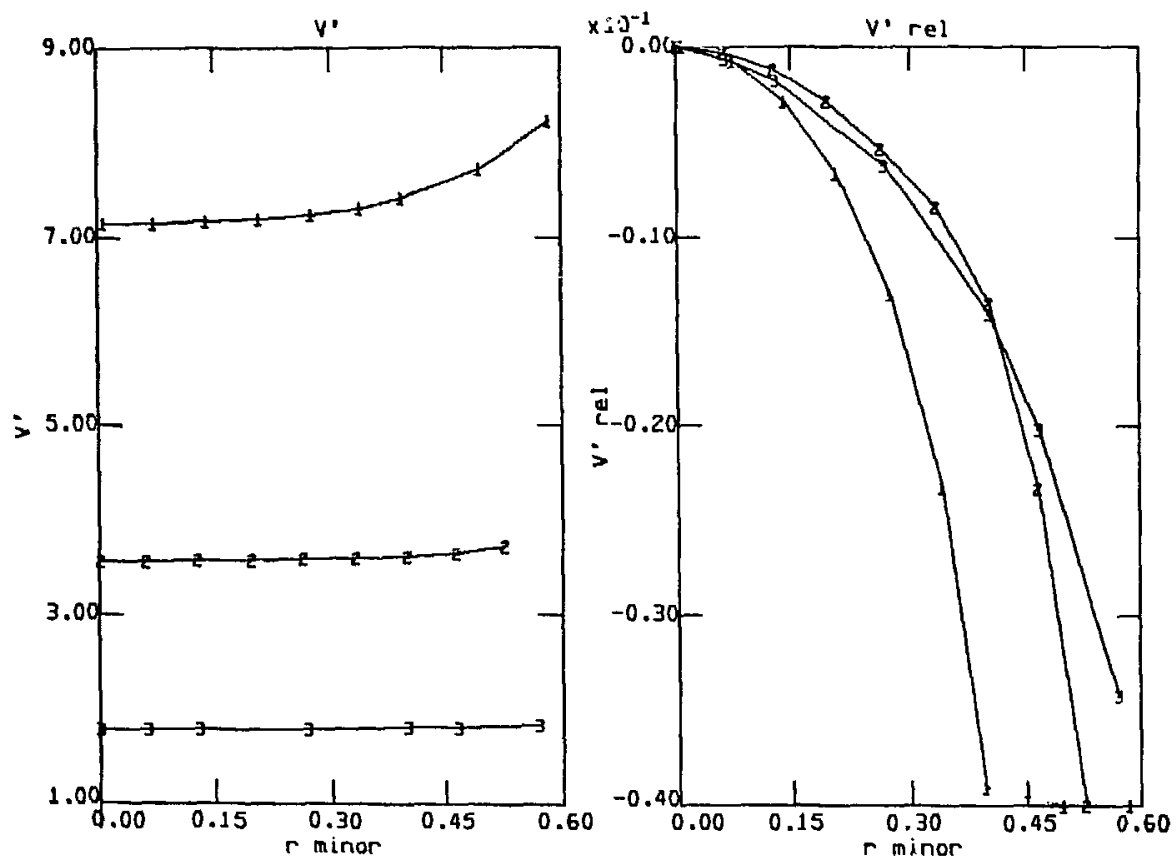

Fig. 14a. $V$ and $V^{\prime}{ }_{r e l}$ : Effects of Number of Coils per Field Period on Magnetic Profiles, Rotational Transform, and Ripple ( $B_{\text {vert }}=2 \% B_{\text {tor }}$ +5 degrees tilt).

Curve 1: 6 Coils/Field Periad,

Curve 2: 12 Coils/Field Period, and

Curve 3: 24 Coils/Field Period.
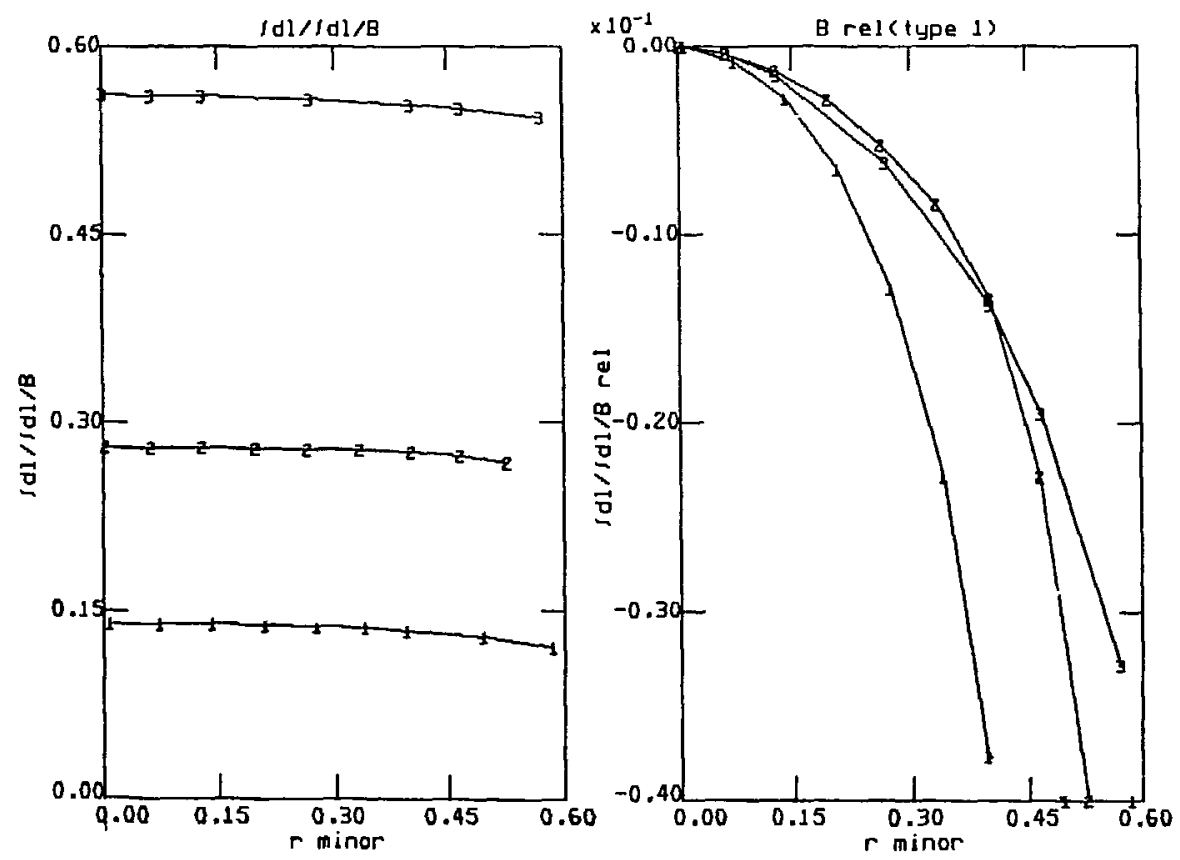

Fig. 14b. <B> and $B_{\text {rel }}$ (type 1) (otherwise, same as Fig. 14a). 

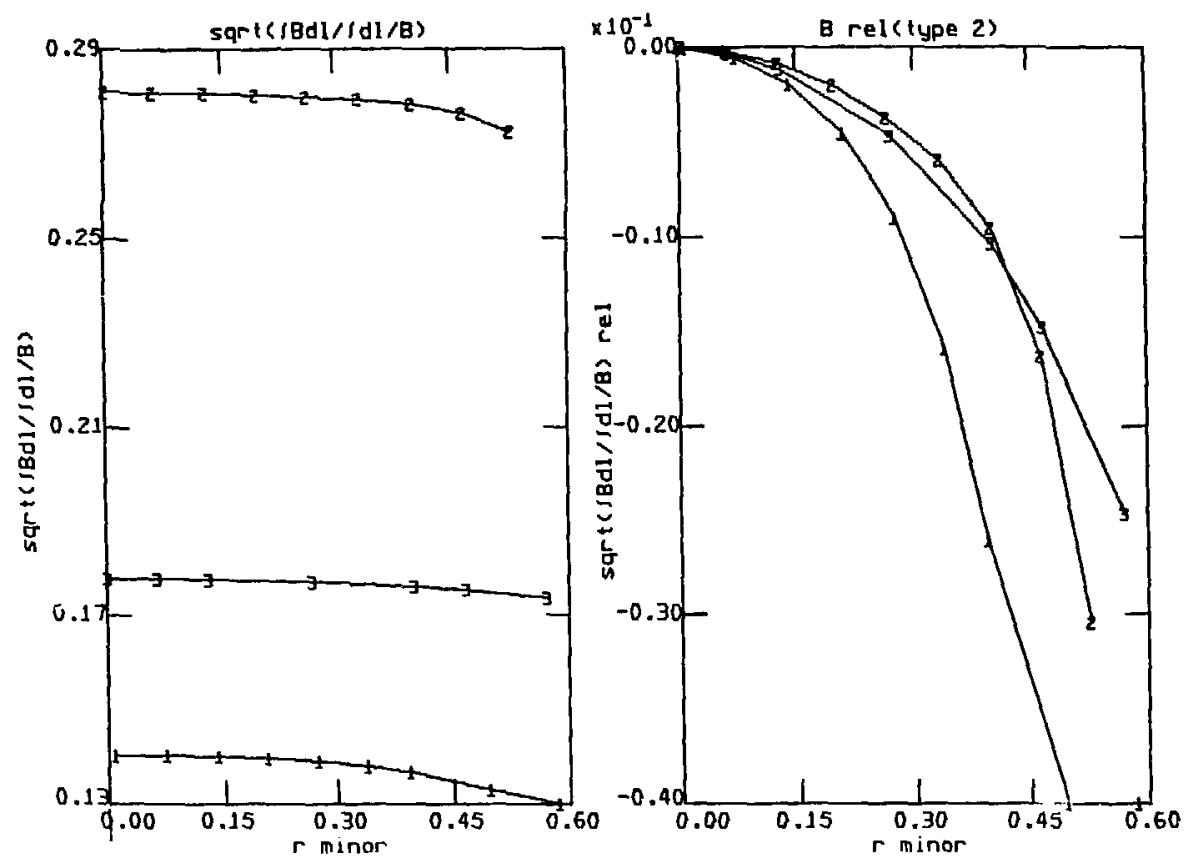

Fig. 14c. $\left\langle B>\right.$ and $B_{r e]}$ (type 2) (otherwise, same as Fig. 14a).
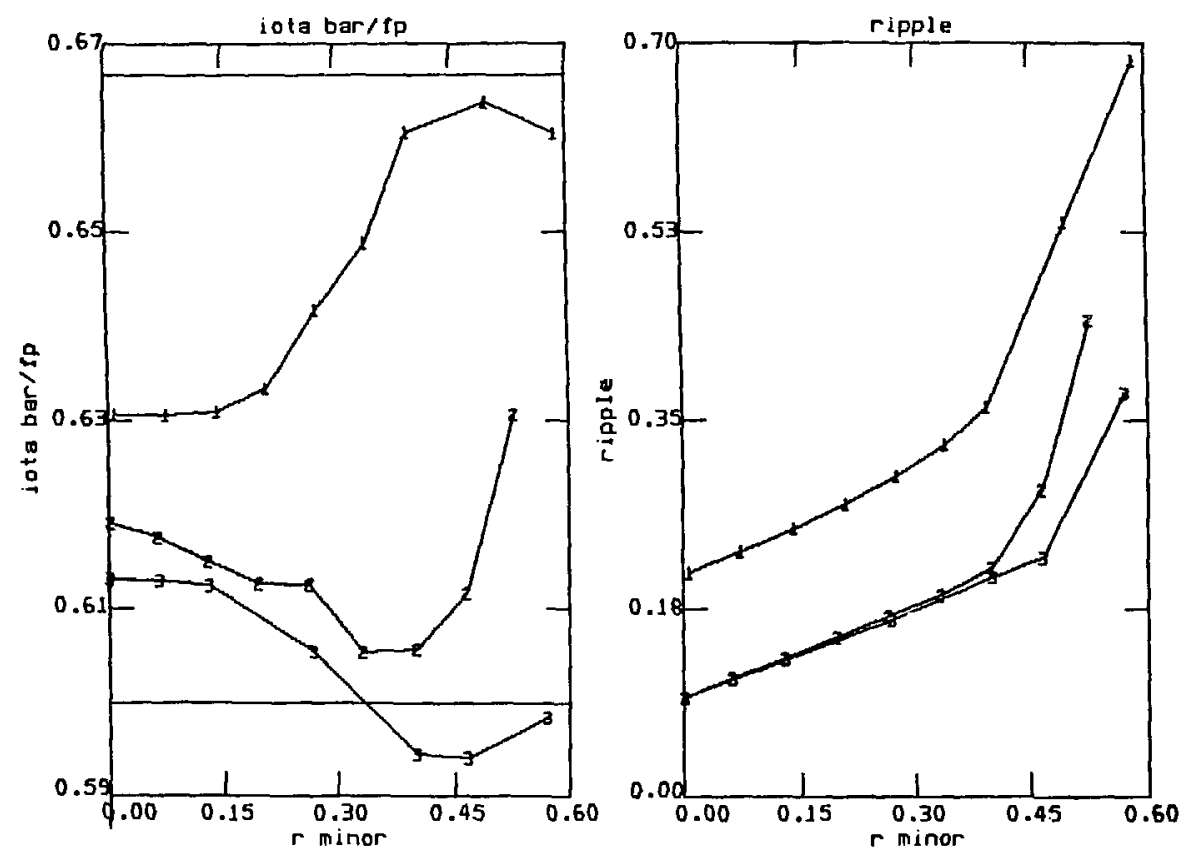

Fig. 14d. Iota Bar per Field Period and Ripple. (otherwise, same as Fig. 14a). 

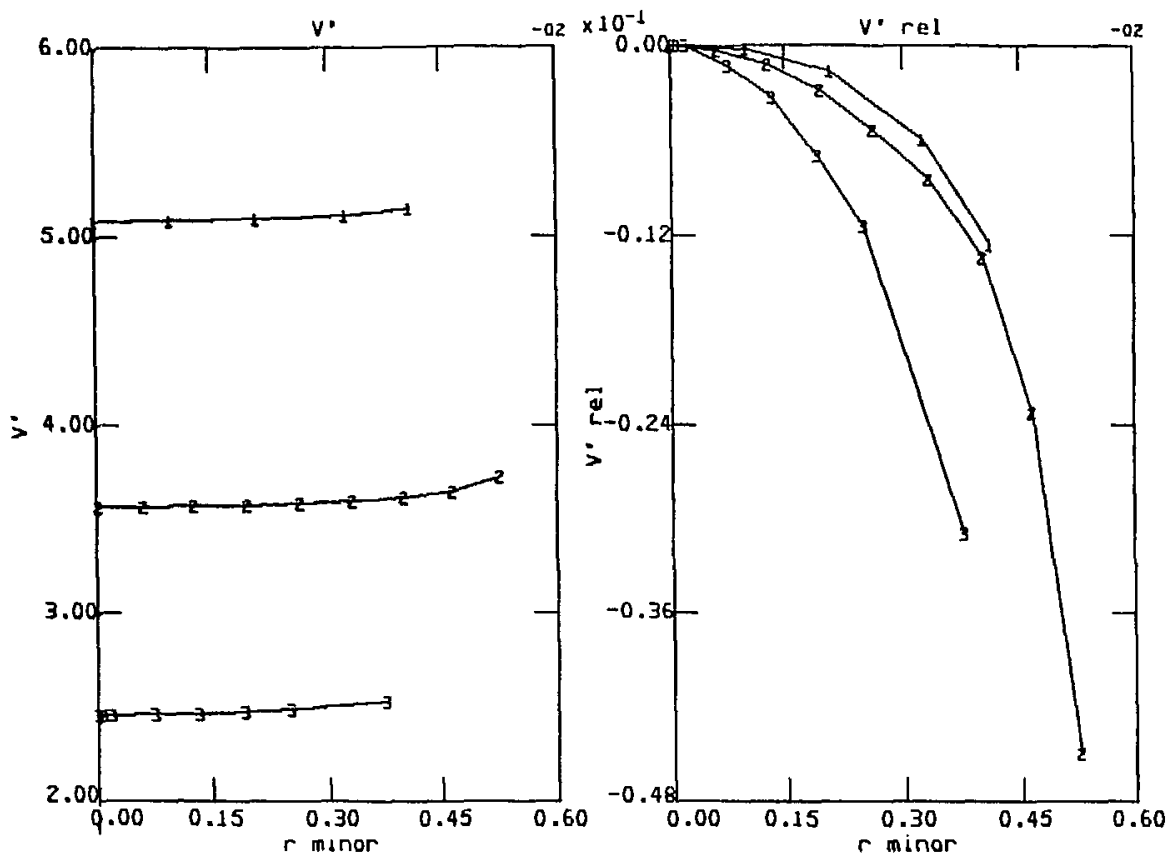

Fig. 15a. $V^{\prime}$ and $V^{\prime}$ rel: Effects of Aspect Fatio at 12 Coils per Field Period on Magnetic Profiles, Rotational Transform, and Ripple $\left(\mathrm{B}_{\text {vert }}=2 \% \mathrm{~B}_{\text {tor }}+5\right.$ degrees tilt - otherwise, same as before).

Curve 1: Major Radius $(a)=8$, Aspect Ratio $(A) \cong B$,

Curve 2. Major Radius $(a)=6$, Aspect Ratio $(A) \cong 6$, and

Curve 3: Major Radius $(a)=4$, Aspect Ratio $(A) \cong 4$.
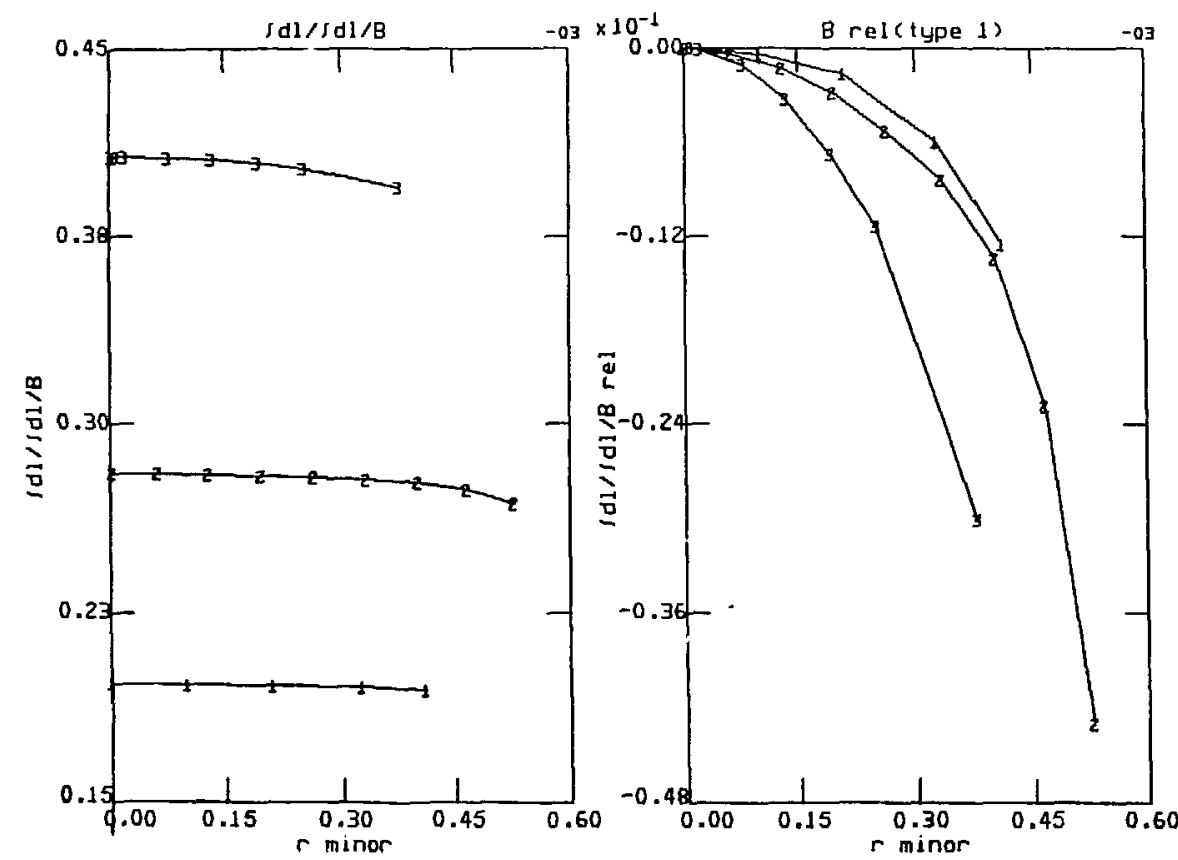

Fig. 15b. $\left\langle B>\right.$ and $B_{\text {rel }}$ (type 1) (otherwise, same as Fig. 15a). 


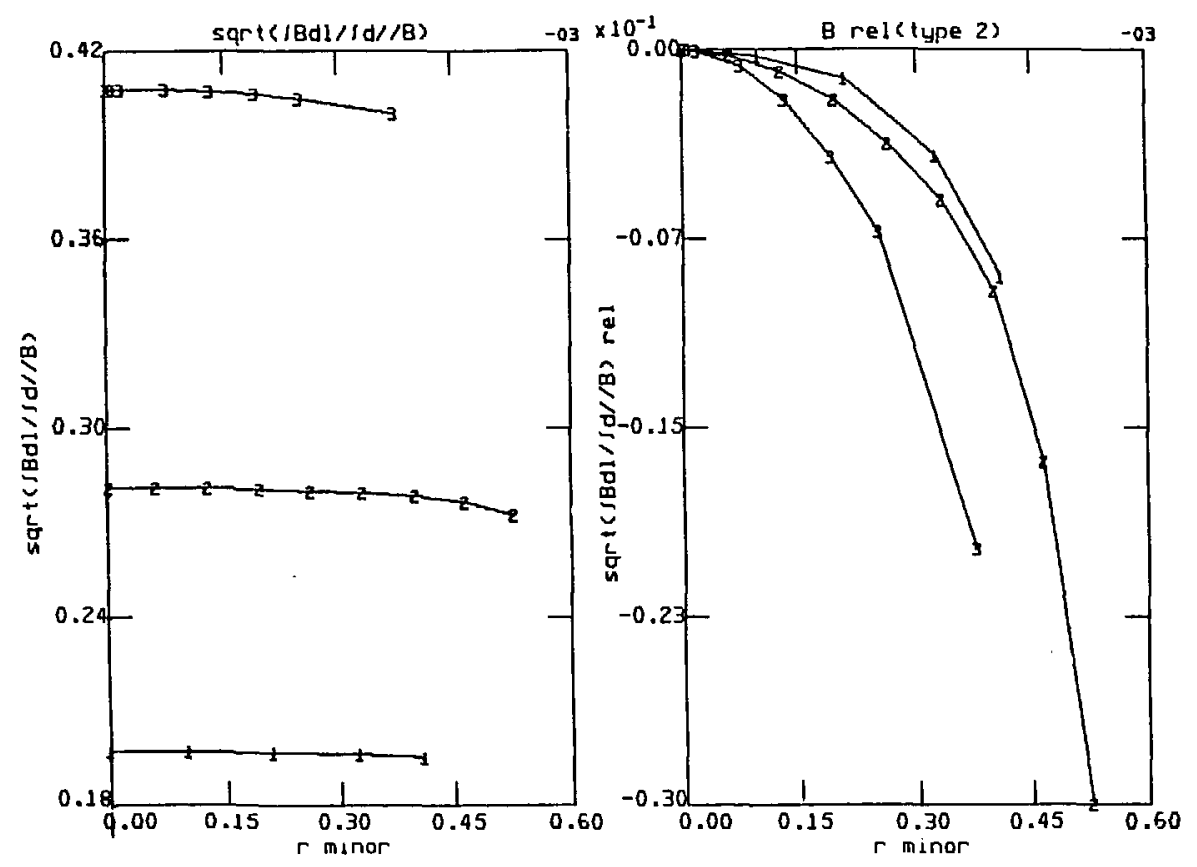

Fig. 15c. $<B>$ and $B_{r e l}$ (type 2) (otherwise, same as Fig. 15a).
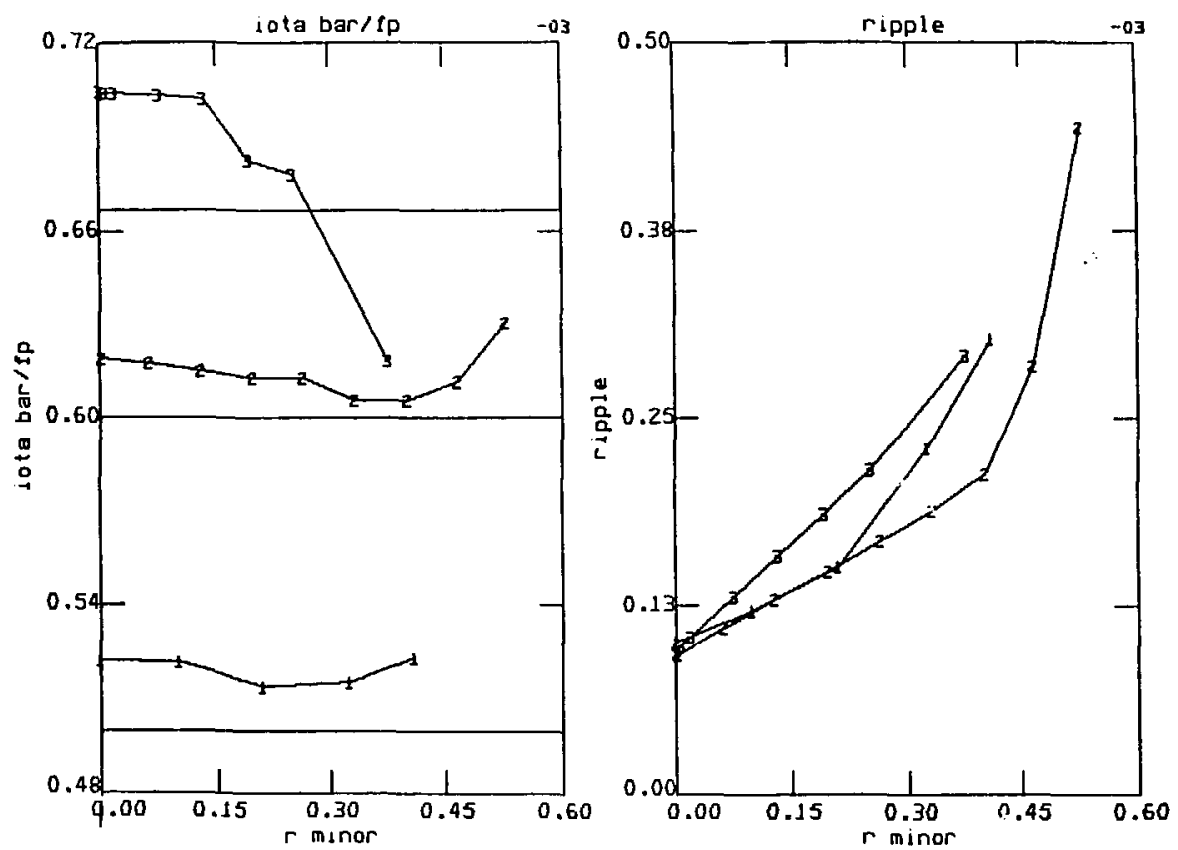

Fig. 15d. Iota Bar per Field Period and Ripple (otherwise, same as Fig. 15a). 


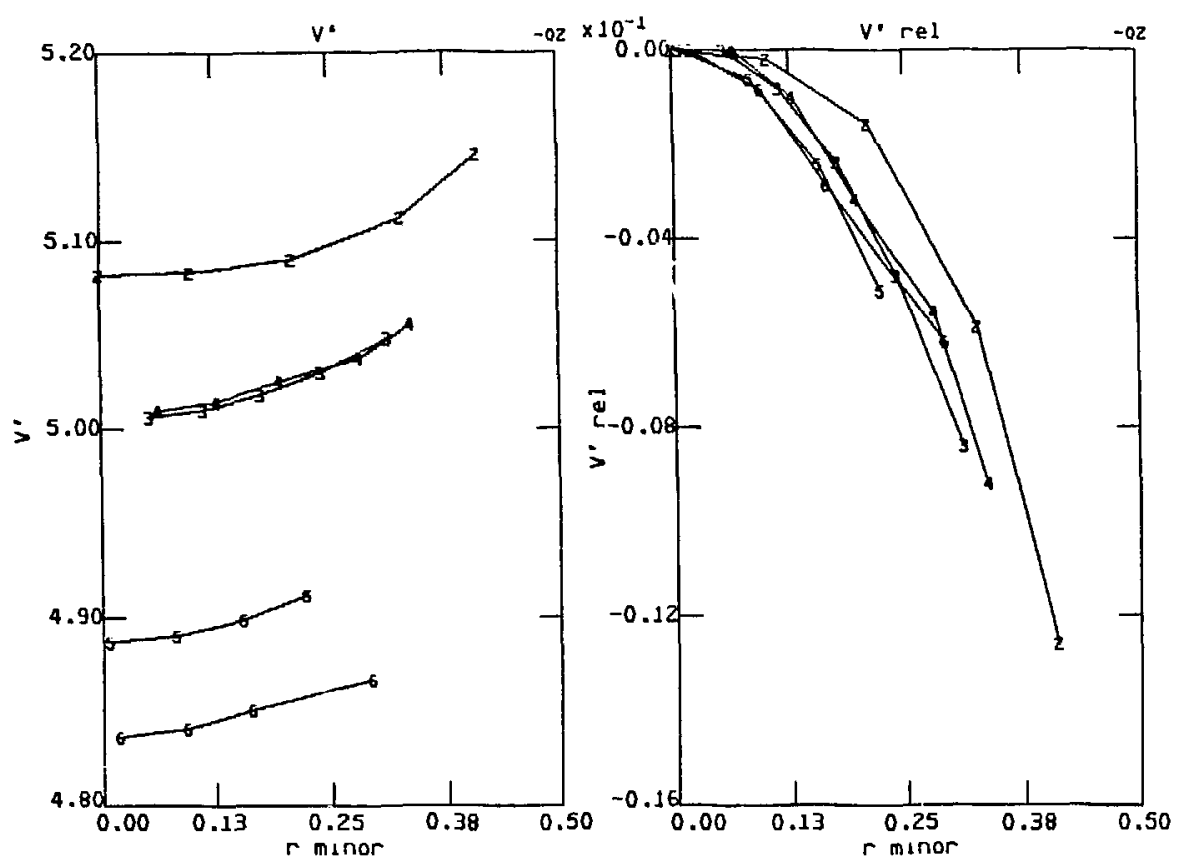

Fig. 16a. $V$ ' and $V^{\prime}{ }_{\text {rel }}$ : Effects of Simultaneous Changes of $C$ and $\Delta_{2}{ }^{*}$ at 11 Coils/Field Period on Magnetic Profiles, Rotational Transform and Ripple ( $B_{\text {vert }}=2 \% B_{\text {tor, }}+5$ degrees tilt).

\begin{tabular}{|c|c|c|c|c|c|}
\hline Curve & $\mathrm{C}$ & $\Delta_{2}{ }^{*}$ & Curve & $\mathrm{C}$ & $\Delta_{2}{ }^{*}$ \\
\hline 2 & $\overline{1.2}$ & $0 . \overline{70}(\mathrm{~h} . \beta . \mathrm{v})$. & 5 & $\overline{1.8}$ & $\overline{0.66}$ \\
\hline 3 & 1.4 & 0.68 & 6 & 2.0 & 0.67 \\
\hline 4 & 1.4 & 0.67 & & & \\
\hline
\end{tabular}
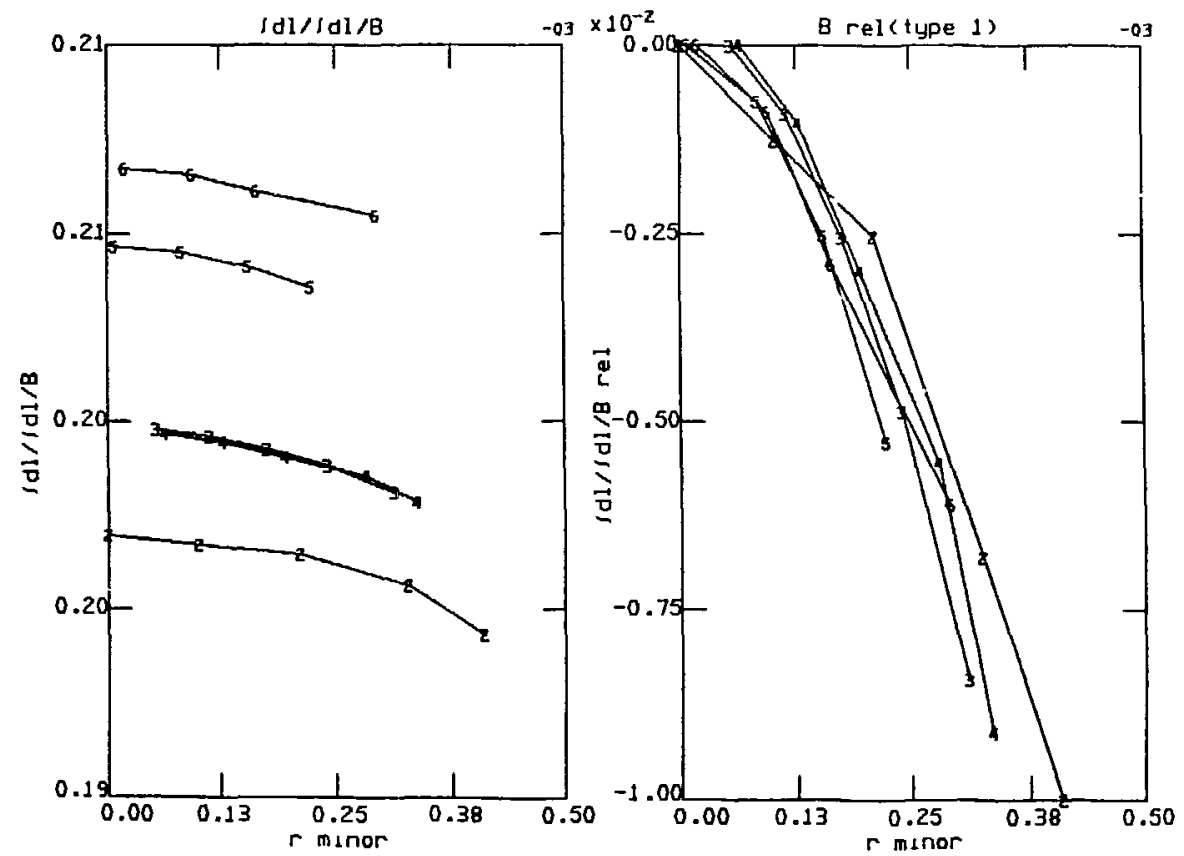

Fig. 16b. <B> and $B_{\text {rel }}$ (type 1) (otherwise, same as Fig. 16a). 


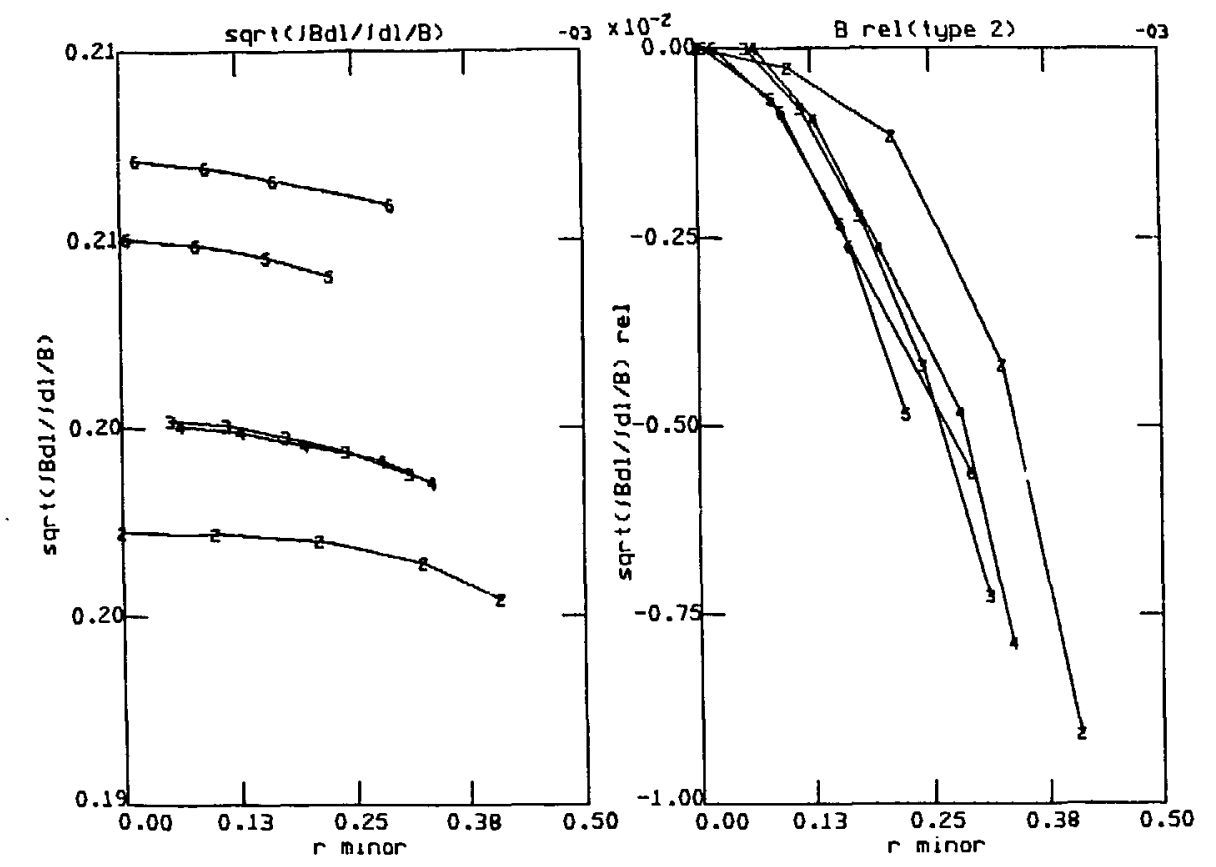

Fig. 16c. $<B>$ and $B_{\text {rel }}($ type 2) (otherwse, same as Fig. 16a).
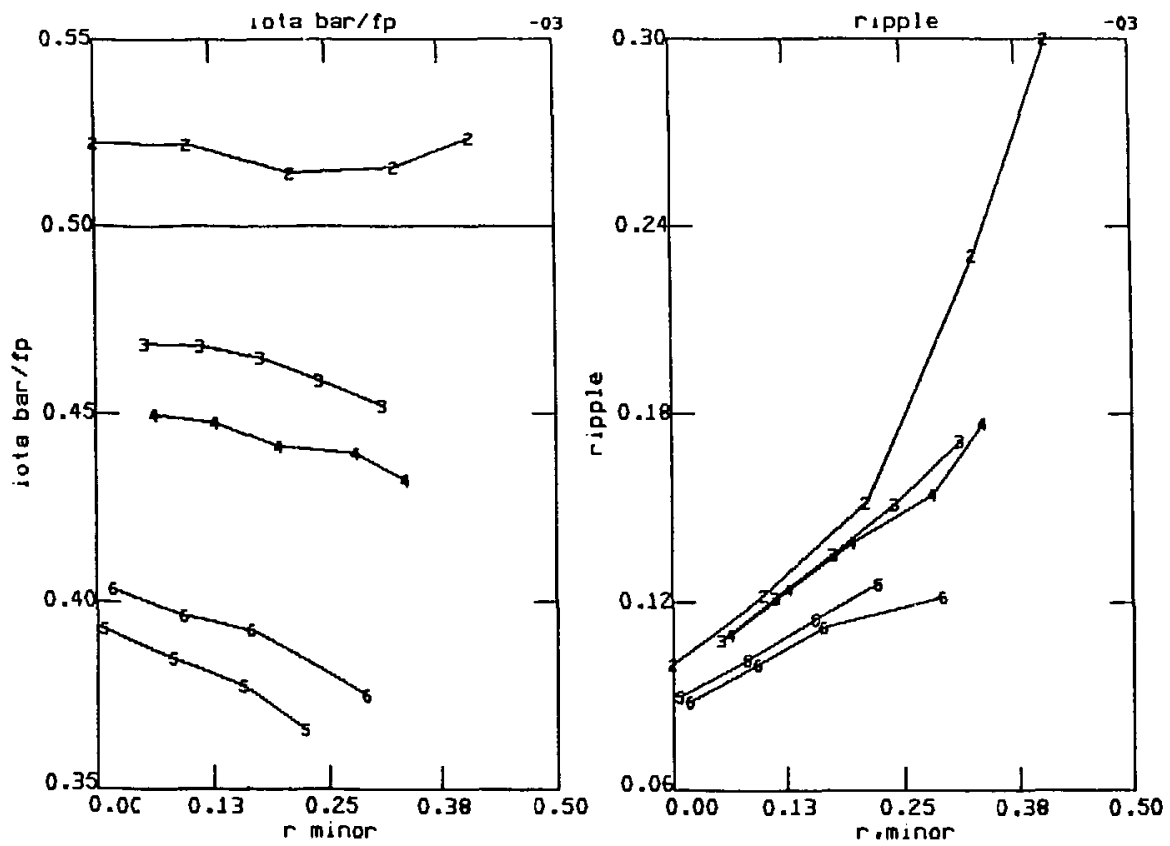

Fig. 16d. Iota Bar per Field Period and Ripple (otherwise, same as Fig. 16a). 